\title{
MECHANISMS OF THE PHOTOTROPIC PATHWAY
}

\author{
A Dissertation \\ presented to
}

the Faculty of the Graduate School

at the University of Missouri-Columbia

In Partial Fulfillment

of the Requirements for the Degree

Doctor of Philosophy

\section{by}

Johanna Morrow

Dr. Emmanuel Liscum, Dissertation Supervisor

July 2018 
The undersigned, appointed by the dean of the Graduate School, have examined the dissertation entitled

\section{"MECHANISMS OF THE PHOTOTROPIC PATHWAY"}

presented by Johanna Morrow,

a candidate for the degree of doctor of philosophy, biology

and hereby certify that, in their opinion, it is worthy of acceptance.

Professor Mannie Liscum

Professor Paula McSteen

Professor Kathy Newton

Professor Melissa Mitchum 


\section{Acknowledgements}

I would like to acknowledge my committee members: Mannie Liscum, Paula McSteen, Kathy Newton and Melissa Mitchum. Thank you for the continued support through these six years and for continuing to challenge me intellectually. Additionally, thank you for the learning opportunities I received, from "rotating" in your lab for 6 months and learning about maize development, to professional skills for graduate students, to the opportunity to be involved in the "All about Plants" outreach at Benton Elementary.

To my boss Mannie, thank you for all the opportunities you have allowed me to pursue while working for you over the years! It has allowed me to (finally) find what I'm passionate about, which is teaching. Not only did I learn from your mentoring and teaching, but you've allowed me to practice this over the years and you have helped point me in the right direction to achieve the best outcome. Thank you for all the support through the years and believing in me! Thanks for all the advice (see I do listen to you)! Over the years you've become more than a mentor and I'm proud to call you friend. It's going to be hard to leave the lab that has been my home for the past almost 17 years!

I would like to thank my family. In particular, I want to say thanks to my wonderful husband Chad, who has always supported me through any and all crazy decisions I have made in this life! Thank you also for having more faith in me than I had in myself at times though this process. It would be negligent of me not to mention the other "members" of our family, Casper, Wendy, Clyde and 
Bonnie, who have also shown unending support and love, in their own way! Thank you to my parents, Sue and John Harris, and my sister Christy Harris, who I can always turn to when I need you.

I would also like to acknowledge current (Kyle Willenburg, Katelynn Koskie, Patrick Nittler, Brittany Cruzan-Johnson, Krista Shucart) and past lab members who have additionally offered insight into my research and lab methods There are so many l've worked with since I started in 2001, that inevitably I'Il miss someone's name, so this is a blanket acknowledgement! I specifically want to acknowledge those whose previous work have made this project possible, especially Diana Robert-Coats, Ullas Pedmale, and Renee Harper. Additionally, thanks to Brittany Cruzan-Johnson and Matt Burger who both worked hard assessing the nph3-16 TILLING allele! First and foremost, thanks for easing all the anxieties (warranted and unwarranted)! Thanks for all the for advice, proofreading, critical insights, laughs, and friendship. I'll always remember my time in the lab fondly, equally as much fun as work and l'll always remember many happy memories. I have made so many good friends through these experiences and I thank and cherish all I've had the pleasure of working with. Through our discussion of politics and science to really bad reality tv, l'll miss this as I go forward!

I need to thank all those in the LSC as well as the Biological Sciences Department for all the help and assistance through the years! I would never be prepared without the help of Nila Emerich! Additional thanks to my amazing mentors Bethany Stone and Amanda Durbak. Thank you also to all in 105 
Tucker, Barb Sonderman in the Tucker Greenhouse, all in 105 and 106 LSC, and Waynes World for all the assistance through the years. Lastly, thanks to all on 3W in the LSC for the comradery and great advice (cloning tips, western blotting advice, stats help, etc.), l'll truly miss coming to work every day! 


\section{Table of Contents}

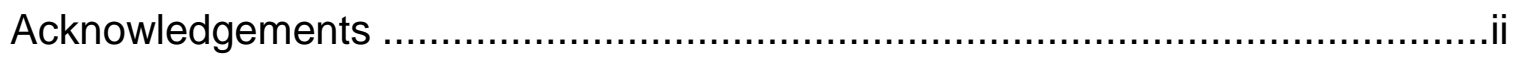

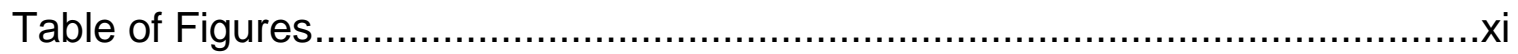

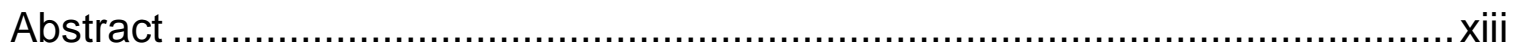

Chapter 1 Literature Review .................................................................. 1

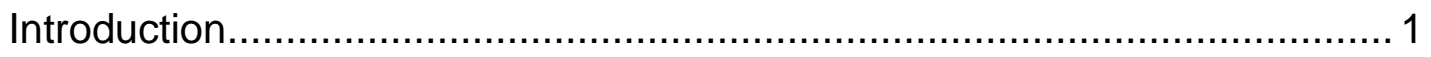

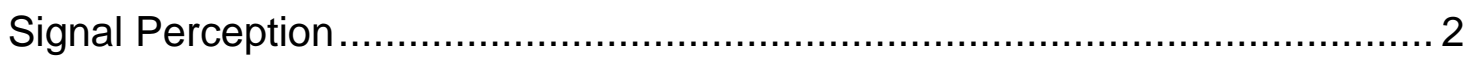

phototropin 1 and 2 are blue light photoreceptors .................................. 2

The phots undergo a conformational change upon blue light perception that

leads to autophosphorylation........................................................... 2

Autophosphorylation of phot1 leads to protein internalization ..................... 4

Functional relevance of phototropin internalization ................................... 5

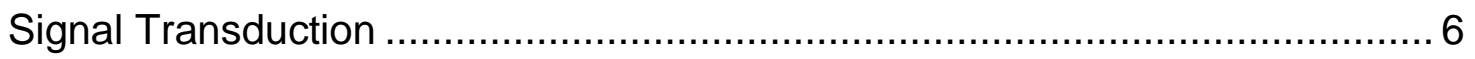

NONPHOTOTROPIC HYPOCOTYL3 (NPH3), a phot-interacting protein, is

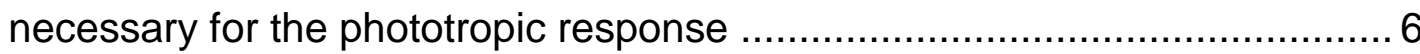

$\mathrm{NPH} 3$ is a phosphoprotein in dark and is rapidly dephosphorylated upon

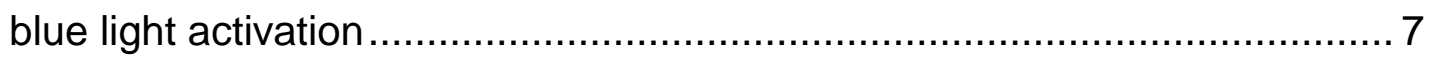

A role for $\mathrm{NPH} 3$ as a substrate adaptor in an E3 ubiquitin ligase complex.... 8 NPH3, along with ROOTPHOTOTROPISM2 (RPT2), are founding members of the NPH3-RPT2-Like (NRL) 33-member protein family .......................... 9 
While the general structures of the NRL proteins are similar, their functions can vary 10

PHYTOCHROME KINASE SUBSTRATE (PKS) proteins are important for the phototropic response and may shed light on the involvement of the redlight photoreceptor phytochrome in this response

Cytosolic calcium is a possible intermediary signal within the phototropic

pathway.

ENHANCED BENDING1 (EHB1), and ADP-RIBOSYLATION FACTOR GTPase-ACTIVATING PROTEIN (ADG12) are NPH3-interacting proteins shown to be involved in the phototropic response 16

A potential role for Heterotrimeric $\mathrm{G}$ proteins in phototropism 17

Protein phosphatases have a variety of functions within phototropic signal transduction

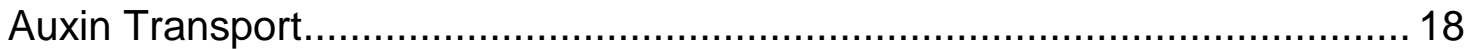

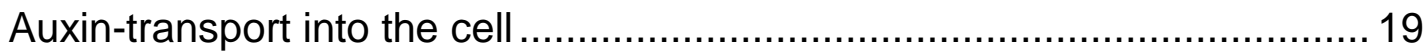

Auxin efflux is facilitated in concert through proteins in two different gene families

Regulation of PIN proteins involves intracellular cycling, phosphorylation and members from the AGC protein kinase family ........................................... 21

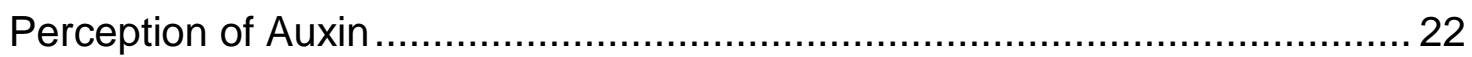

SCFTIR1/AFB functions as an auxin perception complex .......................... 22 
SCF complex proteins: Who they are and what they do

Auxin Signaling: Linking auxin perception to activation

The mechanisms by which the SCFTIR1/AFB complex senses auxin and

activates gene expression

Transcriptional Modification Induced by the Polar Auxin Gradient Leads to

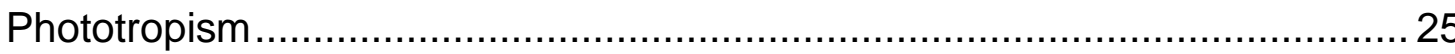

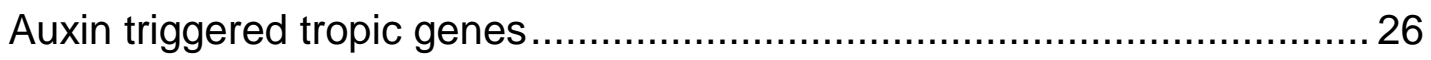

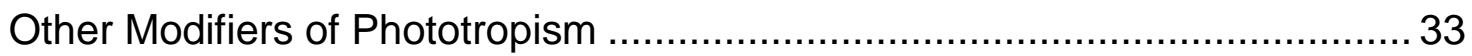

Phytochrome A: Phytochromes are red/far-red reversible light receptors ... 33

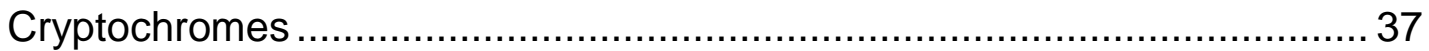

Katanin and microtubule reorientation is necessary for phototropism ..........38

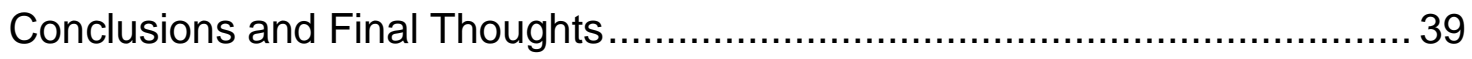

Chapter 2 Characterization of phot 1 function in mutants with altered phot 1

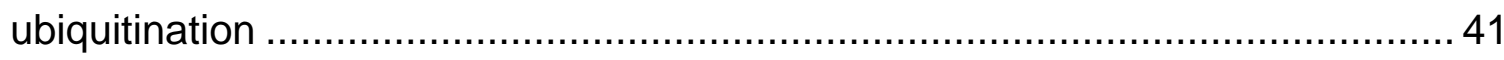

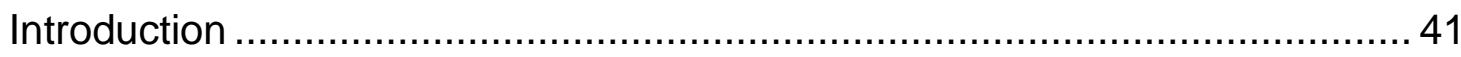

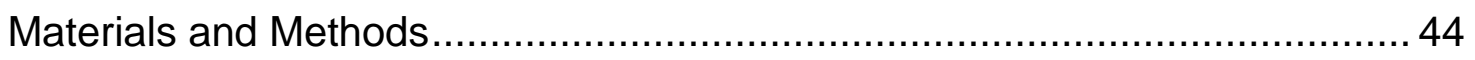

Plant Materials and Generation of Transgenic Plants ............................. 44

Growth and Light Conditions ........................................................... 45

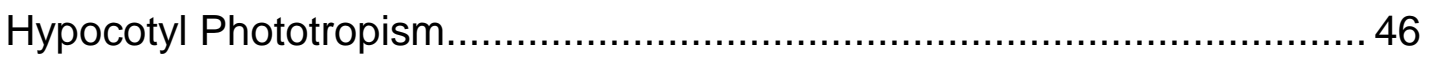

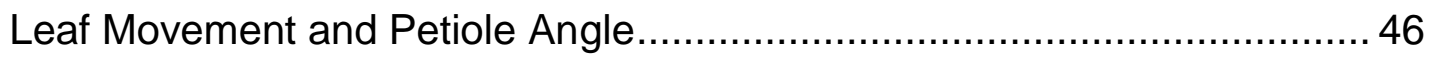

vii 
Chloroplast Movement

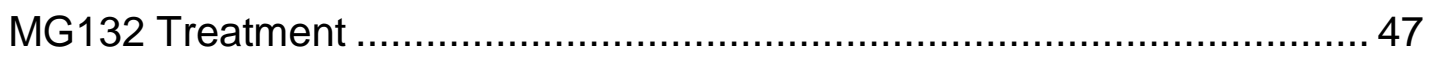

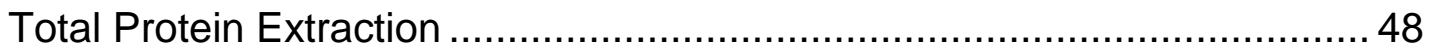

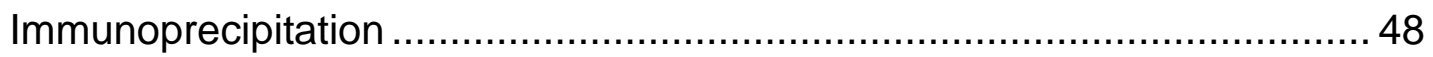

SDS-Page Analysis, Antibodies and Immunoblot Analysis........................ 48

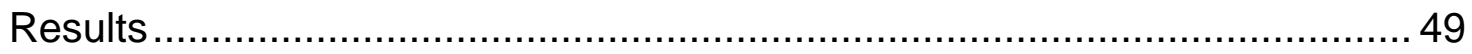

Creation of phot1 Ubiquitin Mutants …............................................... 49

Phototropic Phenotypes of phot1 Ubiquitin Mutants .................................. 54

Leaf Movement Response is Normal in phot1K899R Seedlings ................ 59

Characterization of Another phot1-Mediated Function Which Does Not

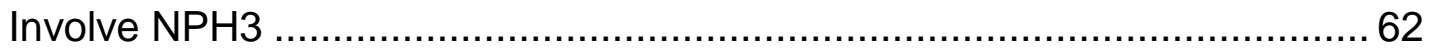

Characterization of phot1 Ubiquitination in Mutant Plants ......................... 68

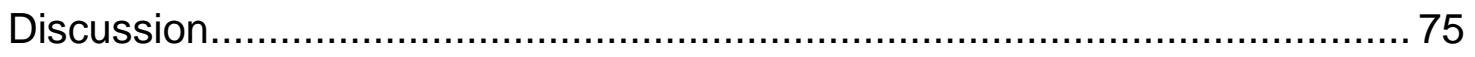

Chapter 3 Characterizing an allelic series of mutants within NPH3 to identify

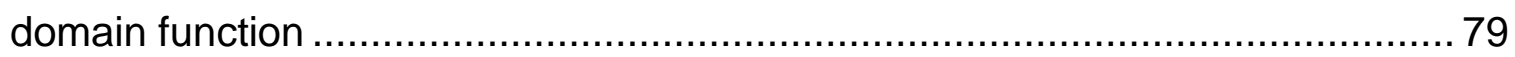

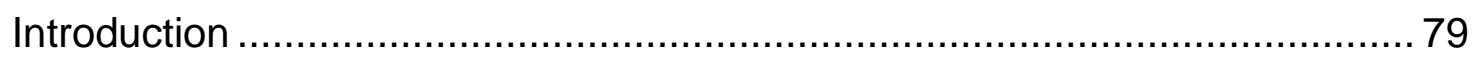

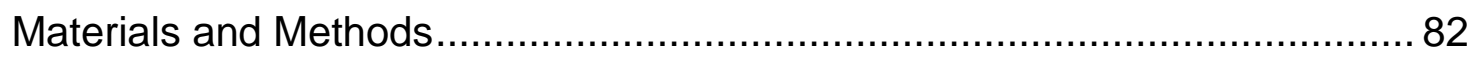

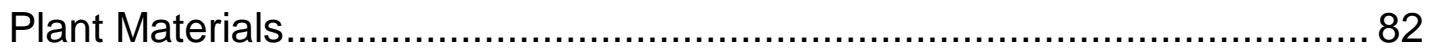

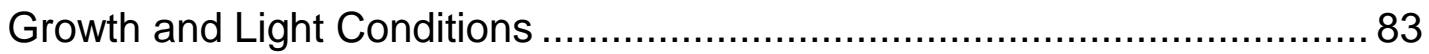

Hypocotyl Phototropism Measurements ................................................. 84 
Leaf Angle Measurements.

Protein Extraction, SDS Page, and Immunoblot Analysis 85

Results 86

nph3 ${ }^{\text {TLLING }}$ alleles Exhibit a Temperature-Sensitive Phototropic Phenotype. 86

Phototropic Phenotypes of $n p h 3^{\text {TILLING }}$ Alleles can be Recapitulated in

Transgenic Seedlings. 91

Leaf Movement in Transgenic TILLING Lines 96

Characterizing the Phototropic Phenotype of Arabidopsis thaliana

Accessions 98

Discussion. 101

Chapter 4 Conclusions and Future Directions 105

Introduction 105

Conclusions and Future Directions 108

Further Characterize Blue Llight-Dependent phot1 Ubiquitination on phot1Mediated Phototropism and Chloroplast Accumulation 108 Identify Phosphorylation Site(s) Within NPH3 Critical to Phototropism ..... 112 Further Characterize NPH3 and Its Role in the CRL3 ${ }^{\mathrm{NPH} 3}$ Complex and phot1 Ubiquitination

References 119 
Vita 


\section{Table of Figures}

Figure 2-1 phot1 complete lysine-arginine mutant express normal levels of phot1

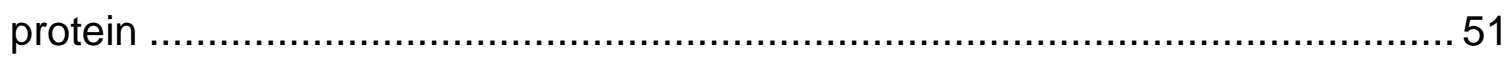

Figure 2-2 Protein expression in phot1K899R seedlings ................................. 52

Figure 2-3 Hypocotyl phototropism of phot1 complete lysine mutant $\left(K^{\star} R\right)$

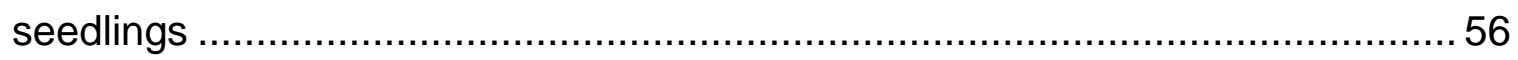

Figure 2-4 Hypocotyl phototropism in phot1K899R ubiquitin mutant seedlings .. 57

Figure 2-5 Hypocotyl phototropism of phot1K526-527R ubiquitin mutant

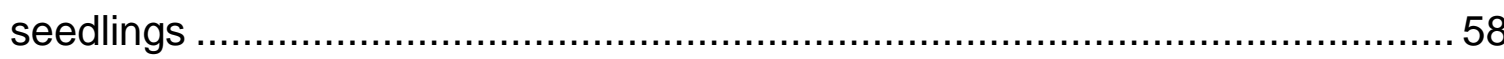

Figure 2-6 Petiole angle measurement example ......................................... 60

Figure 2-7 Leaf movement observed in phot1 K899R mutant seedlings ............. 61

Figure 2-8 Chloroplast movement upon differential light intensity exposure....... 64

Figure 2-9 Chloroplast movement in phot 1 complete lysine mutant seedlings ... 65

Figure 2-10 Chloroplast movement in phot1K899R mutant seedlings...............66

Figure 2-11 Chloroplast movement in phot1K526-527R mutant seedlings......... 67

Figure 2-12 Total protein of mock or MG132 treated low light time course

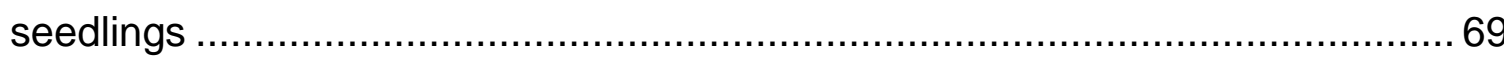

Figure 2-13 Total protein of mock or MG132 treated high light time course

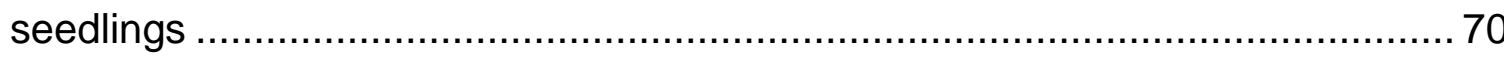

Figure 2-14 Low light phot1 ubiquitination ................................................... 72

Figure 2-15 High light phtot1 ubiquitination ............................................. 74

Figure 3-1 Position of $n p h 3^{\text {TILLING }}$ alleles on block diagram of NPH3 ................. 87 
Figure 3-2 Hypocotyl phototropism of $n p h 3^{\text {TLLING }}$ alleles at room temperature .. 89 Figure 3-3 Hypocotyl phototropism under non-permissive temperature 90

Figure 3-4 Recapitulated TILLING alleles express NPH3 protein...................... 93

Figure 3-5 At room temperature transgenic lines recapitulate phototropic phenotype of original TILLING alleles relative to positive control

Figure 3-6 Hypocotyl phototropism under non-permissive conditions of recapitulated TILLING alleles 95

Figure 3-7 Petiole angle measurements of transgenic TILLING alleles . 97 Figure 3-8 Location of NPH3 SNP within Arabidopsis thaliana ecotypes relative to Columbia 99

Figure 3-9 Hypocotyl phototropism on 3-day-old Arabidopsis thaliana ecotype seedlings 100

Figure 4-1 phot1 protein sequence 111

Figure 4-2 NPH3 protein sequence

Figure 4-3 Preliminary hypocotyl phototropism of Arabidopsis accession lines at non-permissive temperature 118 


\begin{abstract}
Plants are sessile organisms and therefore are limited in their ability to gather resources. Therefore, they have evolved several mechanisms that aid them in their quest to gather light, water and nutrients. One such mechanism is phototropism, a plants ability to bend towards or away from a light source. This mechanism is mediated by the blue light photoreceptor phototropin (phot). Arabidopsis thaliana contains two phototropins, phot1 which is the primary photoreceptor under low intensity light and phot2, which acts redundantly with phot1 under high intensity light. The perception of blue light by the phototropins (phot1 and phot2) initiates signaling events that lead to a lateral redistribution of the plant hormone auxin; which ultimately results in differential growth and the bending response. In addition to phototropism, these proteins mediate several other growth and developmental responses such as leaf movement, chloroplast movement, and stomatal opening. A second protein which is critical to the phototropic response and interacts with phot1 is NONPHOTOTROPIC HYPOCOTYL3 (NPH3). In addition to phototropism, NPH3 has a role in phot1mediated leaf movement. NPH3 has been shown to act as a substrate adapter in an E3 ubiquitin ligase complex with the protein CULLIN3 (CUL3). This CRL3 ${ }^{\mathrm{NPH} 3}$ complex is responsible for ubiquitinating the phot1 photoreceptor in a blue light fashion. This ubiquitination has been shown to be necessary for the bending response, but it's role in receptor ubiquitination is still not fully understood. To better understand this component of phot1-mediated phototropism, we
\end{abstract}


characterized an allelic series of NPH3 mutants to further understand the role of this substrate adapter in this mechanism. Additionally, we characterized several mutant plant lines containing a mutant phot1 protein in which critical ubiquitination sites were mutated in an effort to render the protein unable to be ubiquitinated to further understand phot1 ubiquitination. 


\section{Chapter 1 Literature Review}

By Johanna Morrow and Kyle Willenburg

\section{Introduction}

(Section written by Johanna Morrow)

Plants are sessile organisms. That is, they are rooted in the ground and must make the most out of the environment they find themselves in. Due to this limitation, plants have evolved several mechanisms to optimize their photosynthetic light-capture to help them survive and reproduce. One of these mechanisms is phototropism, a plants ability to bend towards (positive) or away (negative) from a directional light source (Holland et al., 2009). This mechanism is mediated by the perception of blue light by the photoreceptor phototropin (Christie et al., 1998). This response leads to a directional plant growth response in the elongation zone of the stem induced by an asymmetric gradient of the plant hormone auxin, which is known as the Cholodny-Went theory (Went and Thimann, 1937; Esmon et al., 2006). This response has been described by multiple researchers dated back to Darwin in the 1880s, however, steps between blue light perception by phototropin, and bending of the plant organ still need to be elucidated (Liscum et al., 2014). Here we describe current findings within the community related to the phototropic signaling pathway, beginning with signal perception, continuing through signal transduction, as well as the role of auxin transport and perception and ending with other modifiers of the phototropic pathway. 


\section{Signal Perception}

\section{phototropin 1 and 2 are blue light photoreceptors}

The PHOTOTROPIN family in Arabidopsis thaliana contains two members: PHOT1 and PHOT2, and these two genes are present in all higher plants (Briggs \& Huala, 1999), while there has been duplication of these genes in some species (Li et al., 2015). In the context of phototropic function, phot1 has been shown to be the primary photoreceptor under low fluence light, while both phot1 and phot2 act redundantly under moderate to high fluence conditions (Khurana and Poff, 1989; E. Liscum and Briggs, 1995; Sakai et al., 2000; Sakai et al., 2001). In addition to the phototropic pathway, the phots act redundantly in a variety of other functions that impact optimization of photosynthesis. These functions include: stomatal opening, chloroplast accumulation/avoidance, cotyledon/leaf movement, expansion and leaf flattening (Kinoshita et al., 2001; Sakamoto, 2002; Takemiya et al., 2005; Inoue et al., 2008a; Han et al., 2013;

Ohgishi et al., 2004; Sakai et al., 2001); (Jarillo et al., 2001; Kagawa et al., 2001; Sakai et al., 2001).

\section{The phots undergo a conformational change upon blue light perception}

\section{that leads to autophosphorylation}

phots contain an amino-terminal sensory region and carboxyl-terminal output region (Christie et al., 1998). The sensory region contains two LOV (light, oxygen, and voltage) domains, which in darkness each noncovalently binds a Flavin mononucleotide (FMN) chromophore (Huala et al., 1997), while the output region encodes a serine/threonine protein kinase domain (PKD) which is a 
member of the AGC protein kinase family (Bögre et al., 2003; Huala et al., 1997). The LOV domains represent a subset of the Per-Arnt-Sim (PAS) domains that are present in a variety of proteins with diverse functions across a large number of organisms (Rojas-Pirela et al., 2018). The LOV domains were named because the function of the proteins in which they are found are controlled by light, oxygen, and/or voltage (Crosson et al., 2003; Huala et al., 1997). The LOV domains within phototropin have each been shown to have a distinct function: LOV1 is involved in protein dimerization, while LOV2 functions as a molecular light activated switch for repression/de-repression of the PKD (Nakasako et al., 2008; Harper et al., 2004; Jones et al., 2007; Sullivan et al., 2008; Tokutomi et al., 2008; Salomon et al., 2004).

Upon blue light absorption, the FMN chromophore becomes covalently attached to a cysteine residue within each LOV domain, forming an adduct that leads to a progressive conformational change in the protein that moves from the chromophore-binding pocket out to an alpha-helix (the Ja-helix, located in the linker region between LOV2 and PKD). In darkness the protein conformation keeps the PKD in a repressed/inactive state. Blue light-activation leading to the aforementioned conformational changes leads to de-repression of the PKD and allows for protein autophosphorylation/transphosphorylation of the receptor (Christie et al., 1999; Salomon et al., 2000; Inoue et al., 2008a; Sullivan et al., 2008). This autophosphorylation is critical for the phototropic response (Tseng and Briggs, 2010; Inoue et al., 2008a). Within phot1 two serine residues (position S849\&S851) have been identified as critical sites for this 
auto/transphosphorylation; serine851 is critical for phototropism (Inoue et al., 2008a). Within phot2 the phosphorylated serine residues (position S761\&S763) have been shown to be important, but a loss of phosphorylation at these sites does not lead to a complete loss of function as it does in phot1, indicating that there are likely additional phosphorylation site(s) within phot2 that are necessary for the phototropic response (Inoue et al., 2011).

\section{Autophosphorylation of phot1 leads to protein internalization}

Though no transmembrane domain is present, in darkness the phots are associated with the plasma membrane; additionally, the carboxyl-terminal portion of the PKD is required for membrane association (Kong et al., 2013a; b, 2007; Sakamoto and Briggs, 2002). However, upon blue light activation, a portion of the pool of phot protein becomes internalized (Wan et al., 2008; Han et al., 2008; Kong et al., 2006; Sakamoto, 2002). It has been shown that this internalization is dependent upon auto/transphosphorylation in the case of phot1 (Kaiserli et al., 2009; Sullivan et al., 2010). A mutation within phot1 (I608E) leading to a structural change allowing de-repression of the kinase domain renders the protein constitutively active and results in protein internalization in the absence of light. Conversely, no internalization is observed in a phot1 mutant that is phosphorylation-incompetent (D806N), indicating the necessity of auto/transphosphorylation for protein internalization (Kaiserli et al., 2009; Harper et al., 2004). Interestingly, similar to phot1, internalization of phot2 requires the carboxyl-terminal PKD, but phot2 internalization does not require 
auto/transphosphorylation of the protein; blue light activation alone is sufficient (Aggarwal et al., 2014; Kong et al., 2006).

\section{Functional relevance of phototropin internalization}

Protein internalization, in general, has been shown to be involved in the endocytic pathway (Doherty and McMahon, 2009). Indeed, both phots have been shown to interact with clathrin (a coat protein involved in membrane trafficking); phot1 interacts with clathrin heavy chain and phot2 interacts with clathrin light chain (Roberts et al., 2011; Kaiserli et al., 2009; Kong et al., 2006). While it still has not been identified where in the cell phot1 relocates upon internalization, phot2 has been shown to move from the plasma membrane/cytoplasm to the Golgi (Kong et al., 2006; Aggarwal et al., 2014). Interestingly, it has been shown that new microtubules within the hypocotyl are generated in response to blue light stimulation and that this generation of new microtubules is phot-dependent (Lindeboom et al., 2013). Interestingly, plants deficient in Katanin1, a protein that severs microtubules leading to the further growth of the microtubule, have been shown to exhibit reduced phototropic bending (Lindeboom et al., 2013). How this may or may not connect to phot internalization remains unknown.

Though phots can move from the plasma membrane, it is important to ask where phot signaling occurs: the plasma membrane, the cytoplasm, or another compartment/location. It has been shown that seedlings pretreated with red light prior to phototropic blue light stimulation exhibit enhanced responses that are more rapid than in seedlings treated only with blue light (A. Janoudi \& Poff, 1993; a K. Janoudi, Gordon, Wagner, Quail, \& Poff, 1997; Janoudi A-K, Konjevic, Apel, 
\& Poff, 1992). Additionally, phot1 is retained at the plasma membrane in seedlings pretreated with red light, suggesting that phot1 signals from the plasma membrane (Han et al., 2008). More support for plasma membrane associated phot representing the "functional" receptor comes from a study in which phot1 is irreversibly anchored to the plasma membrane via a myristylation/farnestylation tag (Preuten et al., 2015). Seedlings carrying irreversibly-anchored phot1 exhibit a normal bending response; internalization may not be necessary for primary signaling, but it could be involved in receptor turnover or recycling (Liscum, 2016; Preuten et al., 2015; Aggarwal et al., 2014; Kong et al., 2006). Indeed, both phots have been shown to be degraded by the 26S proteasome (Roberts et al., 2011; Aggarwal et al., 2014).

\section{Signal Transduction}

Though the signal transduction pathway from activated phototropin to phototropic curvature has not been reconstructed in its entirety, several components of the pathway(s) have been identified and studied in detail.

NONPHOTOTROPIC HYPOCOTYL3 (NPH3), a phot-interacting protein, is necessary for the phototropic response

$\mathrm{NPH} 3$ was identified in a screen for non-phototropic mutants and has been shown to be critical for the phototropic bending response (Liscum and Briggs, 1996; Okadaa and Shimuraab, 1992; Motchoulski and Liscum, 1999; Liscum, 1995). The NPH3 protein is characterized by an amino-terminal BTB (broad complex, tramtrack, and bric a brac) domain (Stogios et al., 2005), a central NPH3 domain (Pfam, PF03000), and a carboxyl-terminal coiled coil (CC) 
domain (Motchoulski and Liscum, 1999). The CC domain of NPH3 has been shown to facilitate interaction with phot1 (Motchoulski and Liscum, 1999) as well as phot2 (de Carbonnel et al., 2010). In addition to its role in phot-dependent phototropism, NPH3 has been shown to be involved in phot-mediated leaf flattening and leaf movement, but has no role in chloroplast movement or stomata opening (Inada et al., 2004; Inoue et al., 2008b; de Carbonnel et al., 2010).

NPH3 is a phosphoprotein in dark and is rapidly dephosphorylated upon blue light activation

Motchoulski and Liscum (1999) identified an enhanced mobility shift of the $\mathrm{NPH} 3$ protein based on light condition on an SDS-PAGE gel. It was subsequently shown that this light-dependent mobility shift results from dephosphorylation, as this modification could be recreated in the absence of light treatment by application of lambda phosphatase; additionally, treatment with a phosphatase inhibitor blocked the modification induced by blue light (Pedmale and Liscum, 2007). Furthermore, by using pharmacological inhibitors, it was demonstrated that blocking NPH3 dephosphorylation also abrogates phototropic curvature (Pedmale and Liscum, 2007). The dephosphorylation of NPH3, which appears to occur as the result of a yet unidentified type 1 protein phosphatase, has also been shown to be dependent upon the presence of phot1 (Pedmale and Liscum, 2007). The aforementioned observations suggest the phototropically active signaling state of NPH3 is the dephosphorylated form. 
An in-silica phosphorylation site prediction study was performed to identify potential phosphorylation sites within NPH3 implicating 21 sites as phospho-sites (Tsuchida-Mayama et al., 2008). Each of these subsequent predicted phosphorylation sites (serine, threonine, or tyrosine) were mutated to an alanine to render the site phosphorylation incompetent, and the mutants were examined for their phototropic responsiveness. No change in the phototropic nature of plants expressing these mutated phosphorylation sites was observed (TsuchidaMayama et al., 2008), which lends further support to the hypothesis that the dephosphorylated form of NPH3 is the active form of the protein in the phototropic pathway.

\section{A role for NPH3 as a substrate adaptor in an E3 ubiquitin ligase complex}

Previous studies have shown BTB domain-containing proteins can interact with Cullin3 (CUL3) proteins (Genschik et al., 2013). Arabidopsis contains two CUL3 proteins: CUL3A and CUL3B, which are functionally redundant, and a loss-of-function of both proteins results in lethality (Figueroa et al., 2005). However, a cullin3 hypomorph (cul3 ${ }^{\text {hyp }}$ ) was developed as a tool to use in forward genetics studies, which results from a knockout of CUL3B and a knockdown of CUL3A (Thomann et al., 2009). Roberts and colleagues (2011) utilized cul3hyp to ask if CUL3 is involved in the phototropic pathway; the results showed a significant decrease in phototropic curvature while retaining its gravitropic function. These findings indicate that CUL3 proteins are not only involved in the phototropic pathway, but that they likely function early in the pathway because mutations did not impact differential growth generally (Roberts et al., 2011). The 
authors further showed CUL3 co-localizes and interacts with NPH3 at the plasma membrane.

CUL3 constitutes one component of a Cullin Ring Ligase (CRL) complex, a class of E3 ubiquitin ligase complexes that ubiquitinates proteins (Hotton and Callis, 2008; Deshaies and Joazeiro, 2009; Haglund and Dikic, 2005). Roberts and colleagues (2011) were able to show that phot1 is ubiquitinated and this ubiquitination occurs in a NPH3- and blue light-dependent fashion. Given that $\mathrm{NPH} 3$ interacts with CUL3, the authors concluded that NPH3 is functioning as a substrate adapter in a CRL3 complex that targets phot1 for ubiquitination (Roberts et al., 2011). Interestingly, a single ubiquitin or a chain of polyubiquitin molecules may be transferred to proteins in the ubiquitination process, and phot1 exhibits variation in its ubiquitination type depending on the blue light intensity. Phot1 is poly-ubiquitinated in high fluence rate conditions ( $>1 \mu \mathrm{mol} m-2 \mathrm{~s}-1)$, stimulating its degradation by the $26 \mathrm{~S}$ proteasome, while phot 1 is mono/multiubiquitinated under low fluence light ( $<1 \mu \mathrm{mol} \mathrm{m}-2 \mathrm{~s}-1)$ (Roberts et al., 2011). Recent data suggests that the purpose of mono/multiubiquitination is to keep phot1 at the plasma membrane as a signaling competent receptor (Askinosie, 2016).

\section{NPH3, along with ROOTPHOTOTROPISM2 (RPT2), are founding members of the NPH3-RPT2-Like (NRL) 33-member protein family} As previously mentioned, NPH3 is characterized by an amino-terminal BTB domain, a carboxyl-terminal coiled coil domain, and a central conserved sequence-specific region referred to as the NPH3 domain. The NRL proteins all 
contain the NPH3 central region; however, some are missing the carboxylterminal coiled coil domain, and two do not contain the amino-terminal BTB domain (Pedmale et al., 2002). An NPH3 ortholog named COLEOPTILE PHOTOTROPISM1 (CPT1) has been identified by mutation in rice (Haga et al., 2005). Similar to NPH3, a loss of CPT1 function results in a non-phototropic phenotype but retains a normal gravitropic response. Also like NPH3, CPT1 is composed of an amino-terminal BTB domain, a central NPH3 domain, and a carboxyl-terminal CC domain (Haga et al., 2005). While all land plants contain one or more NRL gene, a fewer are found within earlier species (Suetsugu et al., 2016; Christie et al., 2017). Interestingly, Li and colleagues (2011) observed that $\mathrm{NPH} / \mathrm{NRL}$ function often seems to involve a member of the AGC Kinase Family and/or the plant hormone auxin (as will be noted in subsequent sections).

While the general structures of the NRL proteins are similar, their functions can vary

RPT2 is the only NRL member that has been shown to play a role in phototropism (Sakai et al., 2000). In high light conditions where RPT2 function is obvious in the phototropic pathway, it has been shown that response is dependent on the phosphorylation state as well as the localization of NPH3 (Haga et al., 2015; Sakai et al., 2000). RPT2 also interacts with phot1 and has been shown to be involved in leaf flattening, stomatal opening, and chloroplast accumulation responses (Harada et al., 2013; Kozuka et al., 2013; Inada et al., 2004; Suetsugu et al., 2016). 
Recently, another NRL member, NRL31/NCH1 (NRL PROTEIN FOR CHLOROPLAST MOVEMENT 1) was shown to be involved in phot-mediated chloroplast accumulation (Suetsugu et al., 2016). Quite independently, NRL31 was identified as SR1IP1 (AtSR1 interaction protein 1) and shown to play a role in plant immunity (Zhang et al., 2014). Interestingly, NRL31/NCH1/SR1IP1 was shown to interact with CUL3A through its BTB domain and act as a substrate adapter in an E3 ubiquitin ligase complex involved in ubiquitination of AtSR1 in the pathogenesis response (Zhang et al., 2014). Suetsugu and colleagues (2016) also demonstrated that NRL31/NCH1/SR1IP1 interacts with phot1 through its CC domain, much as NPH3 does (Motchoulski and Liscum, 1999).

NRL3 has recently been identified to interact with CUL3A through its BTB domain as well, and was renamed BPH1 (BTB/POZ PROTEIN HYPERSENSITIVE TO ABA 1) due to the nature of the mutant phenotype in response to the plant hormone abscisic acid (ABA) (Woo et al., 2018). While no role in blue light signaling has yet been identified for NRL3/BPH1, Woo and colleagues (2018) speculate that the presence of the NPH3 domain in this gene family member may indeed imply the potential.

NRL20 has been characterized to function in organ development and renamed NAKED PINS IN YUC1 (NPY1)/ENHANCER OF PINOID (ENP1)/MACCHI-BOU4 (MAB4) to reflect mutant phenotypes (Cheng et al., 2007; Furutani et al., 2007; Treml et al., 2005). Four other NRL genes, NRL 6, 7, 21 , and 30 together with $N R L 20$ form a sub-clade of the $N R L$ family (Cheng et al., 2008). Each of these genes have been shown to have overlapping 
expression patterns, and NRL20/NPY1/ENP1/MAB4, NRL7/NPY3, and NRL30/NPY5 have been implicated in organogenesis (Cheng et al., 2008). Interestingly, AGC kinases PINOID (PID), PID2, WAG1 and WAG2, which are in the same subfamily as phot 1 and phot2, have been shown to be involved in NRL/NPY-regulated organogenesis (Christensen et al., 2000; Bennett et al., 1995; Cheng et al., 2008). Each of these AGC kinases appears to regulate auxin responsiveness via NRL/NPY function (Cheng et al., 2008). More recently, the NPY's have been shown to be involved in yet another response mediated by auxin: root gravitropism (Li et al., 2011).

Two additional NRL members have been shown to regulate functions linked to auxin. Namely, NRL23/DEFECTIVELY ORGANIZED TRIBUTARIES3 (DOT3), was identified in a mutant screen for seedlings defective in vein patterning (Petricka et al., 2008). Auxin has been shown to be a key regulator in vascular development (Zhao, 2010). NRL8/SETH6 was also identified in a mutant screen and shown to be involved in pollen germination (Lalanne et al., 2004). Previous studies have shown that auxin plays a role in pollen development (Zhang and ONeill, 1993), which again links the NRL protein function to auxin signaling pathways, that may or may not involve AGC Kinases (Rademacher and Offringa, 2012; Cheng et al., 2008). 


\section{PHYTOCHROME KINASE SUBSTRATE (PKS) proteins are important for the phototropic response and may shed light on the involvement of the red- light photoreceptor phytochrome in this response}

PKS1 was initially identified as a phytochrome-interacting protein that can interact with both the red $(\mathrm{Pr})$ and far-red absorbing (Pfr) forms of phytochrome and negatively regulates signaling (Fankhauser et al., 1999). Additionally, PKS1 is a direct phosphorylation substrate of PhyA (Fankhauser et al., 1999). There are four members of the PKS protein family (PKS1 to 4); both PKS2 and PKS4 have been shown to interact with PHYA (Lariguet et al., 2003; Lariguet and Dunand, 2005; Schepens et al., 2008).

PKS1, PKS2, and PKS4 have all been shown to be important for the phototropic pathway; plants lacking the PKS proteins exhibit a loss-of-function in this response, while double and triple mutants exhibit a greater loss of function phenotype (Lariguet et al., 2006). Studies by Demarsey and colleagues (2012) have shown that PKS4 is a direct substrate of the phot1 kinase domain. The phosphorylation of PKS4 is not a prerequisite for phototropic responsiveness, though it was shown that an increase in phosphorylated PKS4 leads to attenuation of the response (Demarsy et al., 2012). PKS1 has been shown to be a plasma membrane-associated protein in dark and light conditions and to physically interact with both phot 1 and $\mathrm{NPH} 3$, suggesting that these three proteins might form a complex at the plasma membrane (Lariguet et al., 2006). Interestingly, PKS2 also plays a role in leaf flattening and positioning, which is another NPH3-dependent phot-mediated response (de Carbonnel et al., 2010). 


\section{Cytosolic calcium is a possible intermediary signal within the phototropic pathway}

In 1999 Baum and colleagues found that cytosolic calcium levels changed in response to exposure to blue light. Additionally, they demonstrated that this change was phot-dependent (Baum et al., 1999). Using pharmacological compounds, Folta and colleagues (2003) were able to show that blocking calcium influx had an adverse response on the phot1-dependent portion of bluelight-induced hypocotyl growth inhibition. However, these pharmacological experiments did not show a change in the phototropic bending response (Folta et al., 2003). Yet by measuring changes in calcium fluxes, it was shown calcium influx occurs coincident with the phototropic bending response (Babourina et al., 2004). More recently, it has been shown that the influx of calcium is necessary for the bending response in high blue light and that phot2 is required for this response (Zhao et al., 2013).

Increase in cytosolic calcium can come from either the extracellular space or from intracellular compartments such as the vacuole (Sanders et al., 2002). Harada and colleagues (2003) have shown that action of both phot1 and phot2 is needed for blue light induced increase of cytosolic calcium (Harada et al., 2003). However, where the new cytoplasmic calcium comes from varies depending upon the intensity of light. Under low intensity blue light where only phot 1 is active, calcium enters the cell from the extracellular space by means of calcium channels, while in higher intensity light where both phot1 and phot2 are active, 
there is additional influx of calcium from internal stores (Harada and Shimazaki, 2007).

As briefly mentioned before and will be discussed in more detail in the following section, the plant hormone auxin in critical for plant development (Zhao, 2010). It has been shown that upon blue light irradiation, a lateral redistribution of auxin occurs, leading to the bending response (Esmon et al., 2006). One of the auxin transporters involved in this redistribution is PIN1, which is regulated by the protein kinase PINOID (Blakeslee et al., 2004; Friml et al., 2004). Benjamins and colleagues (2003) found that PINOID can physically interact with two proteins involved in calcium binding: TCH3, a calmodulin-related protein, and AtPBP1 (Arabidopsis PID-BINDING PROTEIN1), a calcium binding protein. This finding lends support to a hypothesis that calcium plays a role in the phototropic response (Benjamins et al., 2003). Previous work had already identified the crosstalk between auxin and calcium signaling. In maize coleoptiles, upon unilateral blue light stimulation, a differential gradient of calcium is established across the coleoptile, similar to what has been observed for auxin (Gehring et al., 1990; Felle, 1988). More recently, Zhao and colleagues (2013) have shown that abolishing polar auxin transport adversely affects the bending response in the phototropic pathway and that the amount of IAA and cytosolic calcium are correlated, further supporting the finding that crosstalk between auxin and calcium signaling occurs during phototropism (Zhao et al., 2013). An interaction between PKS1 and Calmodulin4 (CAM4) has been suggested by Zhao and colleagues (2013) as a possible link between auxin, calcium, and phot signaling. 


\section{ENHANCED BENDING1 (EHB1), and ADP-RIBOSYLATION FACTOR GTPase-ACTIVATING PROTEIN (ADG12) are NPH3-interacting proteins shown to be involved in the phototropic response}

EHB1 was identified as an NPH3-interacting protein via a yeast threehybrid assay and confirmed by co-immunoprecipitation (Knauer et al., 2011). Interestingly, ehb1 mutants show an increased phototropic and gravitropic responses. EHB1 was shown to preferentially interact with the BTB domain containing amino-terminal of NPH3 (Knauer et al., 2011). This suggests that EHB1 may compete with CUL3 for binding to the NPH3 BTB domain.

EHB1 contains an amino terminal C2/CalB binding domain, while the carboxyl terminal region shows homology to the ADP-ribosylation factor GTPaseactivating protein (ARF-GAP) family (Rodriguez et al., 2014; Dummer et al., 2016; Knauer et al., 2011). In fact, ADP-RIBOSYLATION FACTOR GTPaseACTIVATING PROTEIN12 (ADG12), shows high sequence similarity to EHB1 and, additionally, contains a C2 domain (Knauer et al., 2011; Dummer et al., 2016). Unlike EHB1, however, adg12 mutants exhibit a loss of function in the phototropic and gravitropic response. Like EHB1, ADG12 has been shown to physically interact with NPH3 (Michalski et al., 2017; Dummer et al., 2016).

Since EHB1 and ADG12 both contain a C2 calcium-binding domain, it was important to determine what, if any, connection exist between these proteins and calcium in the regulation of both gravitropic and phototropic responses. Though exogenous addition of calcium affected both gravitropic and phototropic responses in wildtype seedlings, only the gravitropic response was impacted by 
addition of exogenous calcium to ehb1 and adg12 mutants (Dummer et al., 2016; Michalski et al., 2017). This led the researchers to conclude that phototropic signaling through EHB1 and ADG12 occurs through means other than calcium (Dummer et al., 2016; Michalski et al., 2017).

\section{A potential role for Heterotrimeric $G$ proteins in phototropism}

Heterotrimeric proteins are found in a variety of eukaryotic organisms and consist of three proteins, Ga, $G \beta$, and $G y$ (Urano et al., 2013). These proteins form a complex and are active in a number of signaling pathways (Urano et al., 2013). An A. thaliana $G \beta$ (AGB1) has been shown to physically interact with the amino-terminal region of NPH3 (Kansup et al., 2014). Interestingly, an agb1 lossof-function mutant shows a reduction in phototropic bending response, lending to the hypothesis that it may be involved in this response as well (Kansup et al., 2014). Further studies are however needed to connect $G$ proteins to the overall phot-dependent signaling pathway discussed here.

Protein phosphatases have a variety of functions within phototropic signal transduction

As mentioned earlier, a yet-to-be-identified type 1 protein phosphatase (PP1) appears responsible for the blue light-induced phot-dependent dephosphorylation of NPH3 (Pedmale and Liscum, 2007). Interestingly, mutants defective in RCN1 (ROOT CURLING IN N-NAPHTHYLPHTHALAMIC ACID1), a subunit of type 2A protein phosphatase (PP2A), have been shown to exhibit enhanced phototropic bending as well as increased stomatal opening response (Tseng and Briggs, 2010). RCN1 has been shown to interact with the amino- 
terminal portion of phot2 and dephosphorylate phot2. These results suggest that RCN1 action increases the inactive (phosphorylated) phot2 pool, thereby leading to increased phot2-dependent responses (Tseng and Briggs, 2010). However, rcn1 mutations have shown no impact on phot1-mediated responses (Tseng and Briggs, 2010).

\section{Auxin Transport}

As previously mentioned, the plant hormone auxin (indole acidic acid) is critical in plant development and is involved in many different growth responses. One of these responses involves tropic stimulation. Under natural conditions auxin generated at the tip of the hypocotyl/coleoptile is transported by several different proteins in a polar manner; however, upon tropic stimulation, in addition to polar auxin transport, auxin is laterally transported across the hypocotyl/coleoptile, which has been described in the Chlodony Went Theory (Chlodony and Went, 1937; Ha et al., 2010; McSteen, 2010; Peer et al., 2011).

As the $\mathrm{pH}$ inside the cell is less acidic, as opposed to the extracellular space, auxin can be found either protonated (IAAH) or deprotonated (IAA-) depending on the location. Transport of auxin into cells can occur by naturally diffusing across the membrane as well as with the assistance of the auxin influx carriers AUXIN RESISTANT/LIKE AUXIN RESISTANT (AUX/LAX) proteins (Bennett et al., 1996; Zazímalová et al., 2010). However, auxin needs to be actively transported out of the cell, and this is done with the aid of auxin efflux transporters, the PIN-FORMED (PIN) protein family and ABCB (ATP-binding 
cassette superfamily of transporters, B type/MDR/phosphoglycoprotein (PGP)) (Zazímalová et al., 2010).

\section{Auxin-transport into the cell}

As previously mentioned, due to the $\mathrm{pH}$ difference between the cytosol and the extracellular space, the protonated version (IAAH) of auxin can easily diffuse across the plasma membrane. However, due to the charge of the deprotonated version (IAA-) of auxin, transport is facilitated by use of $\mathrm{H}+$ symporters. These symporters were identified as auxin influx carriers by mutant screens and pharmacological studies (Parry et al., 2001; Bennett et al., 1996). While an agravitropic phenotype has been repeatedly seen in mutants of these genes (Bennett et al., 1996), no change in the phototropic phenotype has been identified. However, Stone and colleagues (2008) demonstrated a role for AUX1 in phototropism that is NPH4/ARF7-dependent. This aphototropic phenotype is not seen in seedlings with normal auxin responsiveness, but in a nph4/arf7 background IAAH diffusion across the membrane is not sufficient influx of auxin within the cell (Stone et al., 2008).

\section{Auxin efflux is facilitated in concert through proteins in two different gene families}

Auxin efflux is facilitated by members from the ABCB (ATP-binding cassette superfamily of transporters, B type/MDR/phosphoglycoprotein (PGP)) (Zazímalová et al., 2010) family, ABCB 1, 4 and 19 to be more specific. Despite the fact that auxin transport happens in a polar fashion (Friml, 2003), these proteins have not been not show to be polarly localized (Cho et al., 2007; Lewis 
et al., 2007). Only mutants deficient in ABCB19 exhibit a phototropic phenotype that differs from wild-type seedling, and, interestingly, the phenotype displayed is a gain-of-function response (Noh et al., 2003; Nagashima et al., 2008). The findings by Christie and colleagues (2011) further link ABCB19 to the phototropic pathway in a study that found upon blue light-stimulation, ABCB19 is a target of phot 1 kinase activity and that this phosphorylation inhibits its auxin efflux activity (Christie et al., 2011). Additionally, in etiolated seedlings it has been shown that ABCB19 inhibits PIN1 cycling, thus keeping PIN1 polarly localized (Titapiwatanakun et al., 2009).

The second family of proteins involved in auxin efflux is the PIN-FORMED (PIN) gene family, named due to the pin-shaped inflorescent phenotype observed in mutant screens (Okada, 1991). The PIN family contains 8 members, consisting of two classes of proteins, the long PINS (PIN1-4 and PIN7) and the short PINS (PIN5-6 and PIN 8). The short PINS have been shown to localize to the ER membrane, whereas the long PINS have been shown to mediate polar auxin efflux in various cell types (Bennett, 2015). Research has shown that PIN1 is primarily polar-localized within the cell as is critical for auxin efflux in shoots, while PIN2 shows similar cellular localization patterns but has been shown to function primarily within roots (Grunewald and Friml, 2010; Liscum et al., 2014). The PINS have been shown to function in many plant growth and development responses like gravitropism and organogenesis, as well as phototropism (Bennett, 2015). In roots Wan and colleagues (2012) have shown PIN2 relocalization in a blue light and phot1-depdendent manner (Wan et al., 2012). 
Additionally, it has been shown that not only do pin3 mutants show a moderate loss-of-function phototropic phenotype, but they show lateral relocalization in response to blue light (Friml et al., 2002; Ding et al., 2011).

\section{Regulation of PIN proteins involves intracellular cycling, phosphorylation and members from the AGC protein kinase family}

Localization and function of the PIN proteins has been shown to involve protein recycling and phosphorylation by two proteins in the AGCV111 protein kinase family. D6 PROTEIN KINASE (D6PK), a member of the AGC 1 protein kinase family, has been identified to be polarly localized and necessary for polar auxin transport through a mutant screen of seedlings lacking the D6PK protein (Zourelidou et al., 2009). Furthermore, Zourelidou and colleagues identified that PIN is a substrate of D6PK kinase activity, resulting in increased PIN activity at the plasma membrane. More recently D6PK has been implicated in phototropic signal transduction as seedlings lacking this protein exhibit a loss-of-function bending response (Willige et al., 2013).

A second kinase involved in regulating PIN function has been shown to be involved in polarity cycling of PIN protein between the apical and basal cell locations named PINOID (PID) (Friml et al., 2004). Two serine/threonine protein kinase, WAG1 and WAG2, have been found to be similar to PID (Santner and Watson, 2006). PID has been shown to phosphorylate PIN proteins, signaling for their polarity switch within the cell (Huang et al., 2010). Interestingly, Ding and colleagues (2011) were able to show that in seedlings lacking PID/WAG1/WAG2, PIN3 localization was unchanged regardless of blue light stimulation. 
Furthermore, these seedlings also exhibited a lack of phototropic response, implicating the involvement of these protein kinases along with PIN proteins in the lateral redistribution of auxin leading to the bending response (Ding et al., 2011).

In addition to cycling between the apical and basal locations within the cell, PIN proteins have also been shown to be recycled by the action of GNOM, which is an ARF GEF (ADP-RIBOSYLATION FACTOR GUANINE-NUCLEOTIDE EXCHANGE FACTOR), recycling PINs from the plasma membrane through vesicle trafficking (Geldner et al., 2003). Seedlings with a partial loss-of-function allele: gnomR5 (Geldner, 2003) display a defect in their phototropic bending response, THUS linking GNOM recycling of PIN proteins to phototropic signal transduction leading to a change in auxin distribution (Ding et al., 2011).

\section{Perception of Auxin}

(Sections below written by Kyle Willenburg)

As described above, phot1 initiates a set of molecular signaling events that lead to the formation of an auxin gradient within the hypocotyl, which is prerequisite to phototropism. In the following sections we will detail the mechanisms by which the auxin perception and response occur.

\section{SCFTIR1/AFB functions as an auxin perception complex}

Auxin perception is necessary to transduce the blue-light dependent auxin gradient into differential growth—phototropic growth. Several systems of auxin perception have been discovered to date, SCFTIR1/AFB complex, S-phasekinase-associated protein 2 (SKP2), and the now more controversial Auxin 
Binding Protein 1 (Strader and Zhao, 2016; Dezfulian et al., 2016; Harper et al., 2000; Grones and Friml, 2015). Due to its specificity the SCFTIRT1/AFB complex, functions as a sensitive receptor of auxin concentration that ultimately leads to signal transduction through activation of specific tropic responsive genes. The SCFTIR1/AFB complex is comprised of three core component proteins: Skp1/ASK1, Cullin1, and an F-box (TIR1 or an AFP) protein, as well as the RBX1 and an E2 ligase proteins necessary for enzymatic activity of the complex (Grones and Friml, 2015). Together, the proteins of the SCF complex function as a nuclear localized E3 ubiquitin ligase to ubiquitinate repressor proteins (Aux/IAAs), marking them for degradation and ultimately leading to transcriptional de-repression of auxin responsive transcriptional factors (ARFs) (Grones and Friml, 2015).

\section{SCF complex proteins: Who they are and what they do}

Arabidopsis SKP1-Like (SKP1/ASK1) functions to connect the SCF complex to the F-box protein. CULLIN1 (Cul1) provides a scaffold for the ubiquitin ligase to associate with, connecting ASK1 and RBX1 proteins together. RING Like BOX PROTEIN 1 (RBX1) connects Cul1 to the E2 ubiquitin ligase. TRANSPORT INHIBITOR RESPONSE 1 (TIR1) and AUXIN SIGNALING F-BOX PROTEINS (AFBs) are F-box proteins that function as coreceptors of auxin along with Aux/IAAs.

\section{Auxin Signaling: Linking auxin perception to activation}

The first component of the auxin responsive signaling response system is the TIR1/AFB auxin co-receptor. The second component is the Auxin/INDOLE-3- 
ACETIC ACID (Aux/IAA) protein auxin co-receptors (Grones and Friml, 2015), and the third the AUXIN RESPONSE FACTOR (ARF) transcription factors that bind to specific DNA sequences, dubbed auxin responsive elements (AuxREs) in the promoters of auxin responsive genes (Ulmasov et al., 2017). There are 29 AUX/IAAs (Kim et al., 1997; Liscum and Reed, 2002; Guilfoyle, 2015), four TIR1/AFBs, and 23 ARFs in Arabidopsis (Okushima et al., 2005). Both ARFs and Aux/IAA transcription factors have been shown to hetero-homodimerize, forming ARF-ARF, ARF-Aux/IAA, or Aux/IAA-Aux/IAA associations by means of electrostatic interactions in their Carboxyl-terminal located PB1 domain, historically referred to as domain III/IV (Korasick et al., 2014; Han et al., 2014; Guilfoyle, 2015). ARFs additionally contain an N-terminal DNA binding Domain (DBD) that recognizes auxin responsive gene promoter regions (Boer et al., 2014; Guilfoyle, 2015).

The mechanisms by which the SCFTIR1/AFB complex senses auxin and activates gene expression

The SCFTIR1/AFB complex functions to ubiquitinate Aux/IAA proteins, targeting them for $26 \mathrm{~S}$ proteasomal degradation. When auxin levels are low, Aux/IAA repressor proteins are bound to ARF transcriptional activators that are associated with (AuxREs), inhibiting transcription of auxin responsive genes (Lavy and Estelle, 2016). However, when auxin concentrations are relatively high, Aux/IAAs bind to auxin in conjunction with the F-box coreceptor TIR1, which in turn allows the E3 ubiquitin ligase complex, SCFTIR1/AFB, to bind the AUX/IAA proteins, polyubiquitinating and marking the Aux/IAA proteins for 
degradation. Once the Aux/IAA proteins are degraded and their ARF binding repression eliminated, ARFs are free to dimerize and induce the expression of the auxin responsive genes they are associated with. The regulatory method of repression/de-repression described above provides a sensitive and elegant mechanism for regulating differential growth.

\section{Transcriptional Modification Induced by the Polar Auxin Gradient Leads to Phototropism}

Auxin-regulated gene expression specific to phototropism requires ARF7 for phototropic signaling (Stowe-Evans et al., 1998)(Liscum, 1995)(Harper et al., 2000)(Liscum and Briggs, 1996)(Liscum, 1995). ARF7 was initially identified as NON-PHOTOTROPIC HYPOCOTYL 4 (NPH4) in a fast-neutron mutant screen (Liscum, 1995; Liscum and Briggs, 1996). At the time NPH4 was not yet characterized as an auxin responsive factor, but phototropic and gravitropic assays soon demonstrated a likely role in modulating the differential growth responses associated with tropic curvature (Liscum and Briggs, 1996).

Phenotypic studies of nph4 mutants suggested that NPH4 functions in the differential growth of tropic curvature and further suggested that NPH4 is a modulator of auxin-dependent differential growth (Stowe-Evans et al., 1998). Map-based cloning of NPH4 revealed that it encodes ARF7 and produced the first genetic links between ARF biochemistry and physiology (Harper et al., 2000). 
In a screen that identified mutants with defects in auxin induced curvature, IAA19/MSG2 was identified (Tatematsu, 2004). In the same study it was shown that NPH4/ARF7 and IAA19 interact with each other and that in the nph4-1 mutant, IAA19 showed a drastic decrease in transcript level, although it normally increases rapidly in response to auxin (Tatematsu, 2004). NPH4 and IAA19 likely function in a negative feedback loop where auxin causes NPH4 to upregulate its own repressor. It is also worth noting that in plants carrying a dominant mutation in IAA19 that stabilizes the AUX/IAA19 protein, a reduction in auxin induced gene expression is observed (Tatematsu, 2004).

\section{Auxin triggered tropic genes}

While research has focused on and generally been effective at elucidating the mechanisms of phototropic light perception and some understanding of the formation of the auxin gradient that precedes and induces the phototropic genes, the specific genes causative for the downstream mediation of the phototropic response have not been fully identified, nor their roles well characterized. The auxin gradient that leads to differential expression of auxin-responsive genes in regions of the hypocotyl necessitates comparing transcripts between opposing flanks of phototropically stimulated hypocotyls to determine which genes are altered by phototropic signaling. This impediment alone would not be a true roadblock if much of the current phototropic research was not conducted in the model plant organisms Arabidopsis thaliana (Esmon et al., 2006). Esmon and colleagues (Esmon et al., 2006) circumvented some of the difficulties associated Arabidopsis thaliana by using Brassica oleracea as a test organism. By collecting 
mRNA from opposing flanks of light-stimulated B. oleracea seedlings and probing an A. thaliana microarray, eight genes were identified that had AuxREs and increased transcript levels where increase in auxin levels was observed (Esmon et al., 2006). What is more, none of the eight genes, termed tropic stimulus induced (TSI) genes, displayed differential accumulation in a nph4-1 null background (Esmon et al., 2006). It is worth discussing each of the genes or gene families in some detail below.

\section{Expansins}

Of the TSI genes identified two genes, EXPANSIN A1 (EXPA1) and EXPANSIN A8 (EXPA8) can be most easily fit into an elegant model for phototropism (Esmon et al., 2006). Expansins promote rapid, within seconds, cell wall creep that allows for cell wall elongation (Yuan et al., 2001; Park and Cosgrove, 2012; Li and Cosgrove, 2001). The expansin superfamily contains four families, 26 alpha expansins (A), 6 beta (B) expansins, 3 expansin-like A, and 1 expansin-like B (Sampedro and Cosgrove, 2005; Li et al., 2002). The proteins themselves are usually 250-275 amino acids long with two domains and a signal peptide to permit its secretion to the apoplast (Yennawar et al., 2006). Expansins are thought to mediate cell growth by functioning to weaken noncovalent bonds that exist between cellulose and hemicellulose, thereby permitting polymers to move as turgor pressure puts tension on the cell wall (Cosgrove, 1997, 2000).

Expansins are an important piece of the acid-growth hypothesis, which states that auxin induces a decrease in $\mathrm{pH}$, leading to subsequent cell-wall loosening (Rayle and Cleland, 1992). Cell wall loosening is highly dependent on 
the $\mathrm{pH}$ of the cell wall with maximal cell wall loosening occurring between $\mathrm{pH} 3.5$ and pH4.5 (Mcqueen-mason et al., 1992; Rayle and Cleland, 1992). It has long been accepted that auxin and cell wall acidity are interconnected (Rayle and Cleland, 1970). More recently, the H+-ATPases (AHAs) have been identified in the process of cell wall acidification (Baxter et al., 2003; Haruta et al., 2010) and the link between auxin and wall acidification more clearly established by the demonstration that auxin stimulates phosphorylation of AHAs (Takahashi et al., 2012). In this context it is important to note that expansin proteins are activated by acidic conditions (Mcqueen-mason et al., 1992). The finding that two expansins (EXPA1 and EXPA8) exhibit increased transcript accumulation in response to tropic stimulation in the elongating hypocotyl flank prior to absorbed curvature early on (Esmon et al., 2006) is certainly consistent with these molecules directly regulating the differential growth response.

\section{Auxin conjugating enzymes GH3.5/WES1 and GH3.6/DFL1 may attenuate auxin induced transcription during tropism}

Signal attenuation is critical for most signaling events, and this is certainly true of phototropism. Because auxin acts to initiate the transcription of genes, either removing auxin or preventing its signaling influences could serve as rapid mechanism to halt such transcription. In plants the GH3 family of proteins have been shown to mediate conjugation of Ala, Asp, Phe, and Trp to auxin as a method of regulating auxin activity (Westfall et al., 2010; Staswick, 2005). Transcripts for two GH3 proteins, GH3.5/WES1 and GH3.5/DFL1, have been shown to accumulate in response to tropic stimulation (Esmon et al., 2006). 
Interestingly, these GH3 transcripts exhibit maximal accumulation coincident with the peak tropic response, suggesting that they may indeed down-regulate the response (Esmon et al., 2006). These two proteins have been shown in Arabidopsis to conjugate amino acids to IAA (Park et al., 2007; Staswick et al., 2002; Staswick, 2005). A dominant overexpressing mutant of the GH3.6/DFL1

gene (dfl1-D) was shown to have reduced shoot elongation in blue, red, and farred light, as well as root phenotypes (Nakazawa et al., 2001). Additionally, GH3.5/WES1 knockout and dominant overexpressing lines manifested interesting phenotypes: the wes 1 knockout showed increased hypocotyl length, while wes1-d dominant-overexpressing line showed decreased hypocotyl elongation compared to wild-type plants (Park et al., 2007). Taken together, current evidence supports a hypothesis for a role of $\mathrm{GH} 3$ proteins in the regulation of hypocotyl elongation, including the differential elongation response observed in tropisms by regulating the auxin concentration fluctuations.

\section{SMALL AUXIN UP RNAs (SAURs) and tropisms}

Among the classes of early auxin responsive genes, SAURs are the largest family (Ren and Gray, 2015). SAURs were originally identified while screening for genes rapidly induced by auxin in soybean hypocotyls (McClure and Guilfoyle, 1987), and present bioinformatic analyses have catalogued 81 SAURs in Arabidopsis (Hagen and Guilfoyle, 2002). At least one SAUR to date (SAUR50) has been shown to be tropically induced in the elongating flanks of growing hypocotyls (Esmon et al., 2006). The SAUR genes usually lack introns (Wu et al., 2012) and have two or more AuxREs (Jain et al., 2006). SAUR genes 
encode small proteins with a predicted range in molecular masses from 9.3 to $21.4 \mathrm{kDa}$ that do not contain any characterized motifs with known biochemical function (Hagen and Guilfoyle, 2002). SAUR proteins are predicted to localize to the nucleus, cytosol, mitochondria, chloroplasts, and on the plasma membrane (Wu et al., 2012). Multiple sequence alignments have revealed that SAUR proteins have a highly conserved central domain (CDD) specific to SAURs that is essential to their function and highly conserved (Marchler-Bauer et al., 2013; Ren and Gray, 2015).

Several SAURs have been implicated in hypocotyl elongation in a number of recent studies (Spartz et al., 2017, 2012; Chae et al., 2012; Qiu et al., 2013; Wu et al., 2012). The method by which SAURs mediate an elongation response is not yet well characterized, but it has been recently suggested that SAURs may be involved in acid growth. For example, SAUR19 appears to upregulate plasma membrane $\mathrm{H}+$ ATPases through a double negative regulation where it inhibits protein phosphatases that are responsible for repression of the $\mathrm{H}+\mathrm{ATPases}$ (Spartz et al., 2014). This finding is particularly exciting knowing that expansins are activated when cell wall $\mathrm{pH}$ is decreased as discussed above. This information together with the finding that SAUR50 is transcriptionally upregulated upon tropic stimulation is indeed intriguing (Esmon et al., 2006).

\section{HATs and auxin-induced hypocotyl elongation}

HAT2 was identified as a tropic stimulus induced gene (Esmon et al., 2006) and has roles that fit into the model of tropic growth so far discussed. HAT2 contains a homeodomain and a leucine zipper domain and is classified as 
a member of the HD-Zip II subfamily which contains 10 members (Ciarbelli et al., 2008; Sawa et al., 2002). HAT2 was shown to be rapidly induced by auxin (within 10 minutes) (Sawa et al., 2002). Interestingly, 35S::HAT2 overexpression lines displayed longer than normal hypocotyls because of increased cell elongation but not cell proliferation (Sawa et al., 2002). Certainly, this phenotype is consistent with differential up-regulation of HAT2 in the elongating flank of a tropicallystimulated seedling being positively correlated with increased elongation in that flank

\section{SKS1 is a Glycosylphosphatidylinositol-Anchored (GPI-Anchored) Protein}

Though identified as a TSI gene, expression of SKS1 was not examined in detail by (Esmon et al., 2006). SKS1 belongs to a 19 member gene family SKS (for SKU5 Similar) which encode structurally related proteins with homology to the multiple-copper oxidases ascorbate oxidase and laccases though they lack copper binding motifs and are thus predicted to confer no catalytic activity (Sedbrook et al., 2002). SKU5, the founding member of the family is a GPI anchored protein (Borner et al., 2003) involved in anisotropic growth, potentially through regulation of cell expansion (Sedbrook et al., 2002; Borner et al., 2003). Though much more research is required into the mechanisms by which SKS proteins function, what little is known implicates SKS1 as a potential player in tropic growth responses. 


\section{A bHLH may serve as an additional regulator of tropic induced hypocotyl elongation}

Previous work has indicated that the expression of a basic helix-loop-helix protein (bHLH) has expression that is induced by both photo and gravitropism (Esmon et al., 2006). bHLH proteins were initially discovered as DNA binding proteins in animals (Murre et al., 1989), then in maize (Ludwig et al., 1989) and yeast (Berben et al., 1990). Within plants bHLH proteins have been shown to be the second largest family of transcription factors (Mao et al., 2017). Most of the recent research conducted on this family has been conducted at the whole genome level (Carretero-Paulet et al., 2010; Li et al., 2006; Pires and Dolan, 2010; Mao et al., 2017) yet few studies have begun to examine the responses these transcription factors regulate. One study showed that PIF3, a bHLH that interacts with the red light photoreceptors phyA and phyB, was involved in regulating phyA and phyb signaling (Ni et al., 1998). More recently it was shown that overexpression of PACLOBUTRAZOL RESISTANCE1 (PRE1)/bHLH134 promotes elongated hypocotyls and early flowering (Lee et al., 2006).

Overexpression of the four most closely related bHLHs to PRE1 (PRE2-5) results in similar phenotypes (Lee et al., 2006). PRE2 is bHLH134, the gene shown to be tropic stimulation induced (Esmon et al., 2006). Further, bHLH134 (called BNQ2 in this study) was shown to heterodimerize with and negatively regulate other bHLH transcription factors, specifically binding to LONG HYPOCOTYL IN FAR-RED LIGHT1 (HFR1) (Mara et al., 2010). All this evidence taken together 
points towards bHLHs involvement in photomorphogenesis and likely in the case of bHLH134, the differential cellular elongation of tropism.

\section{Other Modifiers of Phototropism}

\section{Phytochrome A: Phytochromes are red/far-red reversible light receptors}

phototropin, as discussed so far, is the photoreceptor responsible for perception of blue light and initiates signaling events that progress to the development of a bending response. However, phototropins, though the primary receptor responsible for phototropic light perception, rely on phytochromes, the red/far-red light-sensing family of light receptors to modulate the bending response.

In plants phytochromes are dimerized large, $124 \mathrm{kDa}$, proteins with, broadly speaking, two modules: an $\mathrm{N}$-terminal photosensory module that contains a linear light-absorbing tetrapyrrole and a C-terminal regulatory module important for dimerization and signal output (Franklin and Quail, 2010; Rockwell, 2006; Møller et al., 2002).

Phytochromes are grouped together in a small gene family containing five members PHYA-E (Clack et al., 1994). The five phytochromes (A-E) are divided into two groups: phyA is the sole member of the group I being quickly turned over in high light and serving a primary function in very low fluence responses, group II phys, phyB-C with phyB function as the dominant receptor are light stable and mediate responses such as shade avoidance and flowering time (Fankhauser, 2001). As a group, phytochromes mediate many important responses: germination, de-etiolation, gravitropic orientation, shade avoidance, stomatal 
development, circadian clock entrainment, flowering, and photoperiodic perception (Kami et al., 2010; Franklin and Quail, 2010; Fankhauser, 2001). In addition to these commonly described phytochrome-mediated responses it is well established that phototropic modulation by phytochromes occurs (Parks et al., 1996; Stowe-Evans et al., 2001; Rosler et al., 2007; Hughes, 2013; Lariguet and Fankhauser, 2004). In fact, a number of phytochrome dependent modulations of phototropin signaling have been observed: modulation of chloroplast movement (DeBlasio et al., 2003), far-red-light hypocotyl growth inhibition (Rosler et al., 2007), pulses of red light enhance phototropism phyA dependently (Stowe-Evans et al., 2001; Parks et al., 1996), phyA is necessary for gravitropic abrogation in low fluences of blue light, phyA is necessary for blue light-induced hypocotyl growth inhibition in low to moderate light (Rosler et al., 2007), that both phyA and phyB modulate phototropism without red-light pretreatment, and that phyA functions in the attenuation of phototropism under very high fluence rate phototropism (Whippo and Hangarter, 2004; Tsuchida-Mayama et al., 2010; Kami et al., 2012).

As noted above, phototropin signaling is modulated by the phytochromes in many physiological responses and that phyA seems to serve a primary function in many of the phototropic modulations. Genetic and physiological screening has generally been effective at identifying the modulatory influences of phytochromes on phototropin signaling, but mechanistic evaluation has proven more difficult to elucidate. The localization of phyA and the development of genetic lines that influence phyA localization have proven valuable in describing, 
not the mechanism of action, at least the cellular location. phyA exists as at highly abundant levels in the cytosol in darkness, and upon light perception is quickly shuttled into the nucleus by two proteins FR-elongated hypocotyl 1 (FHY1) and FHY1-like (FHL). In a study by Rosler and colleagues (2007), in a null fhy $1 /$ fhl background where phyA cannot be localized to the nucleus, multiple phyA dependent responses were still observed while several were impaired, which implies that phyA in the cytosol and phyA that is localized to the nucleus both mediate different sets of responses and supports a hypothesis where phytochrome has a cytosolic and nuclear signaling route. It was shown that farred hypocotyl growth inhibition was impaired but not blue-light-induced hypocotyl growth inhibition, implying that phyA cannot signal far-red hypocotyl growth inhibition responses unless it is localized to the nucleus but able to modulate blue-light-induced hypocotyl growth inhibition from the cytosol (Rosler et al., 2007). Interestingly, they further showed that cytosolically localized phyA is able to enhance blue-light phototropism and that seedlings grown in directional blue light are able to abrogate the gravity vector and grow towards a blue light source (Rosler et al., 2007).

It is intriguing to note that many of the cytosolically modulated responses are blue-light phototropin mediated responses. There is evidence to support, though somewhat speculatively, that a more direct signaling route may exist between phot 1 and the phyA. As noted already, phot1 is a plasma membrane associated protein and phyA is cytosolically localized, putting both proteins at least in the same space, making possible some level of interaction, possibly via 
an intermediate molecule. Red-light pulses prior to low fluence rate blue-light treatments has been shown to prevent the blue-light-induced loss of phot 1 from the membrane, a movement with a great deal of research devoted to investigating (Liscum, 2016), but shows that phyA regulates phot distribution (Han et al., 2008). Other evidence exists that phot1 and phyA may cooperate in signaling at the membrane; both phyA and phot1 interact with PHYTOCHROME KINASE SUBSTRATE 1 (PKS1) (Kami et al., 2014; Lariguet et al., 2006;

Fankhauser et al., 1999). Present research has not conclusively demonstrated any more direct interaction between phyA and phot1, but the current evidence is tantalizing nevertheless. When looking in other plant lineages, phots and phys have been clearly shown to interact, so it would be unsurprising to find that a similar relationship exists in higher plants. For instance, ferns and algae mediate phototropism by means of a neochrome photoreceptor (Nozue et al., 1998; Suetsugu et al., 2005; Hughes, 2013), which is essentially a phototropin and phytochrome chimeric protein, in the moss Physcomitrella patens PHY4 was shown to interact with Physcomitrella phototropins in yeast two-hybrid $(\mathrm{Y} 2 \mathrm{H})$ studies (Hughes, 2013). Lastly, (Jaedicke et al., 2012) showed that phot1 and phyA interact at the membrane in onion cells in split-YFP studies, but did not show interaction in $\mathrm{Y} 2 \mathrm{H}$ tests; as a result, the authors suggested that yeast cells may lack one or component of a larger complex, suggesting PKS1 or NPH3 as potential links between phyA and phot1. 


\section{Cryptochromes}

Cryptochromes (crys) are an additional class of blue-light absorbing flavoprotein photoreceptors belonging to a subgroup within the cry/photolyases superfamily, which are broadly distributed from bacteria to plants to humans (Chaves et al., 2011; Liu et al., 2016). The superfamily that crys belong to contains the more well-known proteins, the photolyases, which are responsible for repair of UV induced pyrimidine-pyrimidine DNA adducts by using blue light as an energy source for the repair (Sancar, 2004; Chaves et al., 2011); cryptochromes themselves have no photolyase activity but have protein sequence homology. In Arabidopsis, there are two cryptochromes (cry1 and cry2) that have been implicated in many developmental responses during the lifecycle of plants such as photoperiod induced flowering time (Ahmad et al., 1998; Chaves et al., 2011; Liu et al., 2016), but first discovered for their role in regulating hypocotyl growth inhibition in blue-light (Ahmad and Cashmore, 1993).

Crys facilitate physiological responses to blue through transcriptional regulation of many genes (Franklin et al., 2005; Liu et al., 2011; Chaves et al., 2011). Crys function by perceiving blue light and transducing the signal through other unknown intermediate proteins by means of a C-terminus domain, though the domain is not highly conserved between Arabidopsis cry 1 and 2 (Chaves et

al., 2011). Two methods by which crys regulate transcription have been discovered so far; crys have been shown to operate by suppressing CONSTITUTIVE PHOTOMORPHOGENIC 1 (COP1)-mediated proteolytic degradation of the LONG HYPOCOTYL 5 (HY5) transcription factor and by 
binding directly to basic helix-loop-helix transcription factors (Liu et al., 2012, 2011, 2016).

A number of studies have demonstrated cryptochrome roles in phototropism by evaluating lines expressing single or double mutations and in hypocotyl elongation (Whippo and Hangarter, 2003; Lascève et al., 1999;

Tsuchida-Mayama et al., 2010; Ohgishi et al., 2004; Nagashima et al., 2008; Liu et al., 2016). Cry1, cry2, phyA, and phyB mutants showed defects in high light phototropism and transcriptional activation of RPT2, suggesting that crys together with phy $A$ and phyB may be regulating phototropism by modulating RPT2 expression, which has been shown to attenuate a negative function in hypocotyl phototropism (Tsuchida-Mayama et al., 2010; Inada et al., 2004). Additionally ABCB19 has been shown to suppress phototropism and be regulated by cryptochromes and phytochromes (Nagashima et al., 2008), representing another route that cryptochromes use to regulate phototropism.

\section{Katanin and microtubule reorientation is necessary for phototropism}

In many organisms, centrosomes act to organize microtubule arrays by controlling and positioning nucleation complexes, yet higher plants lack centrosomes altogether and the mechanism by which they regulate microtubule arrays is not well understood (Bartolini and Gundersen, 2006). At the cell cortex, higher plants construct highly ordered microtubule arrays that organize cell wall and growth, directing morphogenesis (Baskin, 2001; Paredez et al., 2006). The microtubules of cortical cells are dynamically responsive to signals, most interesting to this review, light (Zandomeni and Schopfer, 1993). In elongating 
hypocotyls, cortical microtubule arrays are transversely organized to the axis of growth, but blue light signals them to reorient $90^{\circ}$ within minutes (Nick et al., 1990). This blue light induced microtubule reorientation is phototropin dependent, mediated by a Katanin activity, and without Katanin, phototropism is severely impaired (Lindeboom et al., 2013). Do to the severity of phototropic impediment in Katanin mutants, the regulation of Katanin by phot 1 is certainly a critical step in the phototropic signaling and further research in the mechanisms of blue-light dependent microtubule arrangement will likely prove valuable to the field as a whole.

\section{Conclusions and Final Thoughts}

The following sections written by Johanna Morrow

Since Darwin first observed oat coleoptiles bending towards the light in the 1880s, many phototropic signaling events have been discovered. However, the complete pathway between light perception and response - plant bending, still has not been completely elucidated. And as evidenced here, may not be quite as simple as this pathway involves multiple proteins many of whom can affect this signaling pathway in a multitude of means depending on environmental conditions.

$\mathrm{NPH} 3$ structure and function still needs to be fully resolved. Identifying critical phosphorylation site(s) within this protein necessary for the bending response will be helpful in more fully understanding the role NPH3 plays in this pathway. Additionally, as previously mentioned, all $33 \mathrm{NRL}$ family members 
contain the NPH3 domain, but the function of this domain is unknown (Pedmale et al., 2010). Further studies to uncover the function of the NPH3 domain will be helpful, not only to NPH3 and the phototropic pathway, but also to the other 32 members of this family and the growth and development functions they are involved in. NPH3 has been shown to be involved in the blue-light-, CRL3 ${ }^{\mathrm{NPH} 3}$ dependent ubiquitination of phot1 (Roberts et al., 2011). As Roberts and colleagues (2011) have shown, phot1 is ubiquitinated upon blue light exposure, and this ubiquitination is necessary for the bending response. However, the role of phot1 ubiquitination has yet to be fully resolved. Answering the above questions will help to more fully elucidate signal transduction within the phototropic pathway. 


\section{Chapter 2 Characterization of phot 1 function in mutants with altered phot 1 ubiquitination}

\section{Introduction}

Plants are sessile organisms; therefore, they are limited in their ability to gather resources to aid them in their growth and development. As such, they have evolved mechanisms to maximize photosynthetic light capture, as well as water and nutrient acquisition (Lariguet and Dunand, 2005). These mechanisms utilize proteins called photoreceptors (Lariguet and Dunand, 2005).

Photoreceptors are contained in most organisms, plant and animal alike, and are characterized as proteins that are associated with a light absorbing cofactor or chromophore (Parihar et al., 2016). Photoreceptors in plants mediate many growth and developmental processes (Sullivan and Deng, 2003). Of interest to this study are the blue light-absorbing phototropins (phots), which mediate phototropism, leaf movement and expansion, chloroplast accumulation and avoidance, and stomatal opening (Christie et al., 2015). Arabidopsis thaliana contains two phots, phot1 and phot2 (Lariguet and Dunand, 2005).

Phototropin is characterized by an amino-terminal sensory domain and a carboxyl-terminal protein kinase (PK) output domain. The sensory domain

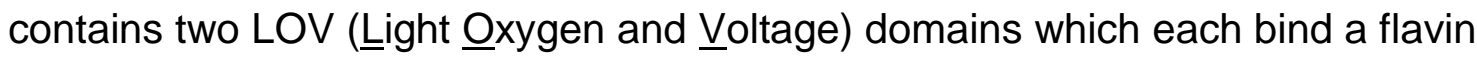
mononucleotide (FMN) chromophore (Huala et al., 1997). In darkness these FMN chromophores are bound non-covalently, while upon blue light exposure they form a covalentadduct with conserved cysteines. In response to adduct 
formation, phots undergo a conformational change that leads to PK activation and subsequent autophosphorylation/transphosphorylation, (Christie et al., 1998; Salomon et al., 2000; Sullivan et al., 2008; Inoue et al., 2008a). The phots are plasma membrane-localized in darkness, however, upon blue light exposure, a portion of the total phot protein pool is internalized (Sakamoto and Briggs, 2002; Kong et al., 2007). Of particular interest to this study is the mechanism phototropism, a plants ability to bend toward or away from a light source. Arabidopsis contains two phots: phot 1 functions as the primary photoreceptor under low intensity light, while phot1 and phot2 act redundantly in high intensity light (Lariguet and Dunand, 2005).

A second protein which has been shown to be critical for phot1-mediated phototropism, as well as phot1-mediated leaf movement, is NPH3

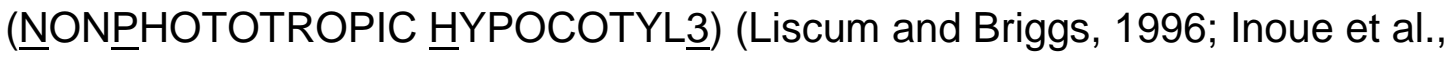
2008b). NPH3 is characterized by an amino-terminal BTB (broad complex, tramtrack, and bric a brac) domain (Stogios et al., 2005), a central 'NPH3 domain' (Pfam, PF03000), and a carboxyl-terminal coiled coil (CC) domain (Motchoulski and Liscum, 1999; Pedmale et al., 2010). The CC domain of NPH3 has been shown to mediate interaction of NPH3 with phot1 in light and dark conditions (Motchoulski and Liscum, 1999).

$\mathrm{NPH} 3$ is a phosphoprotein which is dephosphorylated upon blue light exposure in a phot1-dependent manner by a yet to be identified type 1 protein phosphatase (Pedmale and Liscum, 2007). In fact, Pedmale and Liscum (2007) demonstrated that it is the dephosphorylated state of the protein which is likely 
the active form for phototropic bending to occur. Additionally, the BTB domain of NPH3 has been shown to mediate interaction with CUL3 (CULLIN3). As such, NPH3 acts as a substrate adapter in a CULLIN RING E3 ubiquitin ligase complex (CRL3 ${ }^{\mathrm{NPH} 3}$ ) that is responsible for ubiquitinating phot1 in response to blue light exposure (Roberts et al., 2011) Roberts and colleagues (2011) demonstrated that under high intensity blue light phot 1 is polyubiquitinated by $C R{ }^{\mathrm{NPH}}$, resulting in protein degradation by the $26 \mathrm{~S}$ proteasome. In contrast, under low intensity blue light phot 1 is mono/multiubiquitinated. Further studies were performed to identify K899 as the critical blue light- and NPH3-dependent ubiquitination site within phot1 (Roberts, 2013). This K899 site is targeted for both mono/ and polyubiquitination (Roberts, 2013). This study, and independently one by Deng and colleagues (2014), identified a ubiquitination site at $\mathrm{K} 526$ which is blue light- but not NPH3-dependent.

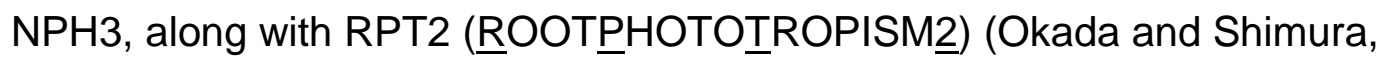
1992; Harada et al., 2013; Sakai et al., 2000) are the founding members of a 33-

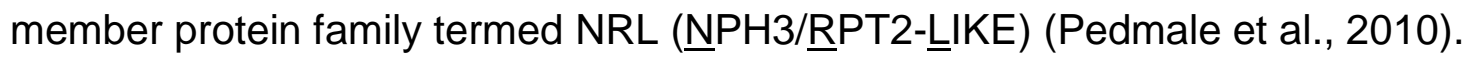
Members of this protein family are involved in a variety of processes such as phototropism, chloroplast movement, organ development, pollen germination, vein patterning, and pathogen responses (Suetsugu et al., 2016; Zhang et al., 2014; Petricka et al., 2008; Cheng et al., 2007; Lalanne et al., 2004). The general structure of the NRL proteins is similar as they all contain the central ' $\mathrm{NPH} 3$ domain', two thirds of the family contain the CC domain, but most strikingly, all but two contain the amino-terminal BTB domain (Pedmale et al., 2010). 
Furthermore, two members of this family: NRL31/NCH1 (NRL PROTEIN FOR CHLOROPLAST MOVEMENT 1)/ SR1IP1 (AtSR1 interaction protein 1) and NRL3/ BPH1 (ㅁTB/POZ PROTEIN HYPERSENSITIVE TO ABA 1) have both been shown to interact with CUL3 through the BTB domain (Zhang et al., 2014; Woo et al., 2018).

While ubiquitination of phot 1 has been shown to be necessary for phototropic responsiveness (Roberts et al., 2011), the mechanism by which ubiquitination influences the response is not yet fully understood. phot 1 mutants were generated by using site-directed-mutagenesis substituting a lysine(s) (identified to be blue light and/or NPH3-dependent) with an arginine(s).This substitution conserves the structure and charge of the normally-occurring amino acid, but unable to be modified with the ubiquitin molecule (Haglund and Dikic, 2005). The purpose of this study is to identify what effect(s) the inability to be ubiquitinated, completely or at individual identified lysines, has on phot1mediated processes.

\section{Materials and Methods}

\section{Plant Materials and Generation of Transgenic Plants}

Positive controls used in these experiments are the Arabidopsis thaliana Columbia (Col-0) accession, as well as transgenic phot1-GFPphot1-5 which expresses phot1 tagged to GFP transformed into a phot1-null mutant in Col-0 (Sakamoto and Briggs, 2002). The phot1-5 mutant is used as a negative control (Liscum and Briggs, 1995). 
phot1 ubiquitin mutants (substituting an arginine for the normally occurring lysine) were generated by site-directed-mutagenesis (Thermo Fisher Phusion Site-Directed Mutagenesis Kit), thus prohibiting the ubiquitin molecule from binding to that site. Additionally a cDNA with all 80 lysines converted to arginine was synthesized to render phot1 incapable of being ubiquitinated (Diana Roberts \& Liscum, E, 2013; Life Technologies). Expression of PHOT1 transgenes were controlled by the native phot1 promoter, as well as 3' UTR, cloned into a binary vector containing GFP (Curtis and Grossniklaus, 2003). We utilized the floral dip method (Clough and Bent, 1998) to mobilize constructs into the null phot1-5 mutant. Homozygous independent insertion lines identified by utilizing a hygromycin screen were used for further experiments.

\section{Growth and Light Conditions}

Seed was sterilized with $30 \%$ bleach for 30 minutes and plated on half strength Murashige and Skoog (MS) media plates with a thin layer of media on top. Plates were then placed in the cold at $4^{\circ} \mathrm{C}$ for a period of $2-4$ days for the purpose of vernalization. After vernalization, seed was exposed to red light for 1 hour to stimulate uniform germination as described by Stowe-Evans and colleagues (2001). Experiments were then performed on seedlings which were grown in darkness for three days following red light treatment. Unless otherwise stated in following experimental design, seedlings for total protein extractions were either mock treated or exposed to saturating blue light conditions (3000umol m²) (Lascève et al., 1999) 


\section{Hypocotyl Phototropism}

Three-day-old seedlings were exposed to 4 hours of unilateral blue light at a fluence rate of $0.1 \mu \mathrm{mol} \mathrm{m} \mathrm{m}^{-2} \mathrm{~s}^{-1}$. After light treatment seedlings were placed on transparency film, covered with transparent tape. Curvatures were determined from tracings of immobilized seedlings (Stowe-Evans et al., 2001). Data was plotted as average degrees of curvature plus and minus standard error. A students T-test was used to determine statistical significance of means between genotypes in a given condition.

\section{Leaf Movement and Petiole Angle}

Seed was sown on promix with additional Miracle-Gro water soluble allpurpose plant food (https://www.miraclegro.com) (1 tbsp per gallon of water) added for nutrients, and plants allowed to grow for 9 days under $50 \mu \mathrm{mol} \mathrm{m} \mathrm{m}^{-2} \mathrm{~s}^{-1}$ constant white light. Plants were then transferred to red $\left(25 \mu \mathrm{mol} \mathrm{m} \mathrm{m}^{-2} \mathrm{~s}^{-1}\right)$ plus blue light $\left(0.2 \mu \mathrm{mol} \mathrm{m}^{-2} \mathrm{~s}^{-1}\right)$ for 5 days (Inoue et al., 2008b). Plants were then photographed from the side and 'petiole angle' was measured using FIJI imaging software. Angle measured from hypocotyl to right petiole, with angle vertex serving where the petiole is attached to the hypocotyl, with one ray forming a horizontal line at this attachment and the second moving up along the petiole (Figure 2-6). Data is plotted as mean petiole angle plus and minus standard. Statistical significance was determined by performing a student's T-test between genotypes. 


\section{Chloroplast Movement}

Mesophyll chloroplast positioning within a leaf was determined by assessing a change in red light transmittance through the leaf after specific light treatments, as described by Johansson and Zeidler (2016). Using a hole punch, leaf discs were taken from 3 to 5-week-old rosette leaves, placed in 96 well plates containing half strength MS media topped with 100ul of half strength MS liquid, and then placed in the dark for 3 hours to allow chloroplasts to return to dark state. Using the Nanoquant Tecan Infinite 200 microplate reader, measurements were taken at wavelengths of $660 \mathrm{~nm}$ and $800 \mathrm{~nm}$. Four measurements were taken in the dark with a period of 10 minutes between each measurement. Following dark measurements, 96 well plates containing leaf discs were exposed to low intensity blue light $\left(0.1 \mu \mathrm{mol} \mathrm{m}^{-2} \mathrm{~s}^{-1}\right)$ for 60 minutes with measurements taken every 10 minutes within that 60 -minute time period. After 1 hour of low intensity light, leaf discs were exposed to high intensity blue light (75 $\mu \mathrm{mol} \mathrm{m} \mathrm{m}^{-2} \mathrm{~s}^{-1}$ ) for 60 minutes again with measurements taken at 10 -minute intervals throughout the 60 -minute light treatment.

\section{MG132 Treatment}

To assess ubiquitination in transgenic lines, seedlings were either mock treated with DMSO or treated with $50 \mathrm{ug} / \mathrm{ml}$ of the $26 \mathrm{~S}$ Proteasome inhibitor MG132 (AdipoGen, catalog number AG-CP3-0011) in 5-8 mL's of half strength MS liquid for 2 hours in darkness with gentle shaking. Following treatment, a time course was performed where seedlings were exposed to either low intensity blue 
light $\left(0.1 \mu \mathrm{mol} \mathrm{m} \mathrm{m}^{-2} \mathrm{~s}^{-1}\right)$ or high intensity blue light $\left(117 \mu \mathrm{mol} \mathrm{m}^{-2} \mathrm{~s}^{-1}\right)$ for a time period of 0,30 or 240 minutes.

\section{Total Protein Extraction}

Following the indicated treatments, seedlings were removed from plates and frozen in liquid nitrogen. Protein was extracted by grinding in buffer described by Lariguet and colleagues (2006), followed by centrifugation at $10,000 \mathrm{rpm}$ to remove particulate matter. Supernatants containing total protein were then transferred to a new tube. Protein was quantified by using Coomassie Protein Assay Kit (Thermo Pierce, catalog number 23200).

\section{Immunoprecipitation}

Protein from MG132 or mock treated seedlings were incubated with AntiGFP mAb-Agarose (MBL, D153-8) plus protease inhibitor (Sigma, P9599) for 4 hours on a rotary shaker at $4^{\circ} \mathrm{C}$. After incubation, agarose beads were washed 3 times in protein extraction buffer, $2 X$ Urea buffer added to the sample, and the sample was then boiled for 5 minutes prior to separating on an SDS-Page gel.

\section{SDS-Page Analysis, Antibodies and Immunoblot Analysis}

Total protein samples were separated on an $8 \%$ SDS-Page gel until the 70kDa protein standard contained in the Pageruler Prestained Ladder (ThermoFisher, catalog number 26616) was at or near the bottom of the gel. Separated proteins were then transferred to nitrocellulose by electrophoresis in a $20 \%$ methanol, tris-glycine buffer at $250 \mathrm{~mA}$ overnight at $4^{\circ} \mathrm{C}$. Nitrocellulose blots were then incubated in $5 \%$ dry milk made in tris-buffered saline (TBS) with $0.5 \%$ Tween 20 for a period of at least 2 hours prior to immunodetection. 
Primary antibodies used include previously described anti-phot1 (1:8000 dilution ratio, in 1\% milk, TBST) (Christie et al., 1998), anti-NPH3 (1:6000 dilution ratio, in 1\% milk, TBST) (Motchoulski and Liscum, 1999), and a ubiquitin-specific antibody, P4D1, which detects mono/multiubiquitin, as well as polyubiquitin chains (1:1500 dilution ratio, in 1\% milk, TBST) (Cell Signaling, 3936S). Nitrocellulose blots were incubated with primary antibodies for a period of no less than 2 hours. Secondary antibodies were incubated with blots (goat anti-rabbit, Genscript, catalog number A00098, 1: 10,000 dilution ratio, in 1\% milk, TBST; and goat anti-mouse, Bio-Rad, 170-656, 1:3000 dilution ratio in 1\% milk, TBST) following primary antibody incubation for 1 hour. Chemiluminescent HRP substrate (Millipore Sigma, catalog number WBKLS0500) was used to detect protein.

\section{Results}

\section{Creation of phot1 Ubiquitin Mutants}

Previously identified ubiquitination sites within the phot1 protein (K527 and K899) (Roberts, 2013; Deng et al., 2014) were mutated to assess the physiological impact on phot1 transgenic plants deficient in their ability to be ubiquitinated at K526 or K899. K527 was additionally mutated along with identified K526, as it's been reported that ubiquitination can be promiscuous, attaching a ubiquitin molecule to a near-by lysine if it's intended target is unavailable (Stringer and Piper, 2011). Additionally, plants containing a mutated phot1 protein where all 80 lysines are substituted with arginine (Life Technologies) were evaluated, henceforth referred to as phot $1 K^{\star} R$. Arginine was 
chosen as it mimics structure and charge of lysine but is unable to bind covalently to the ubiquitin molecule (Haglund and Dikic, 2005). Several independent lines were identified for each specific transgene which express mutant phot1 protein (Figure 2-1, Figure 2-2a). 


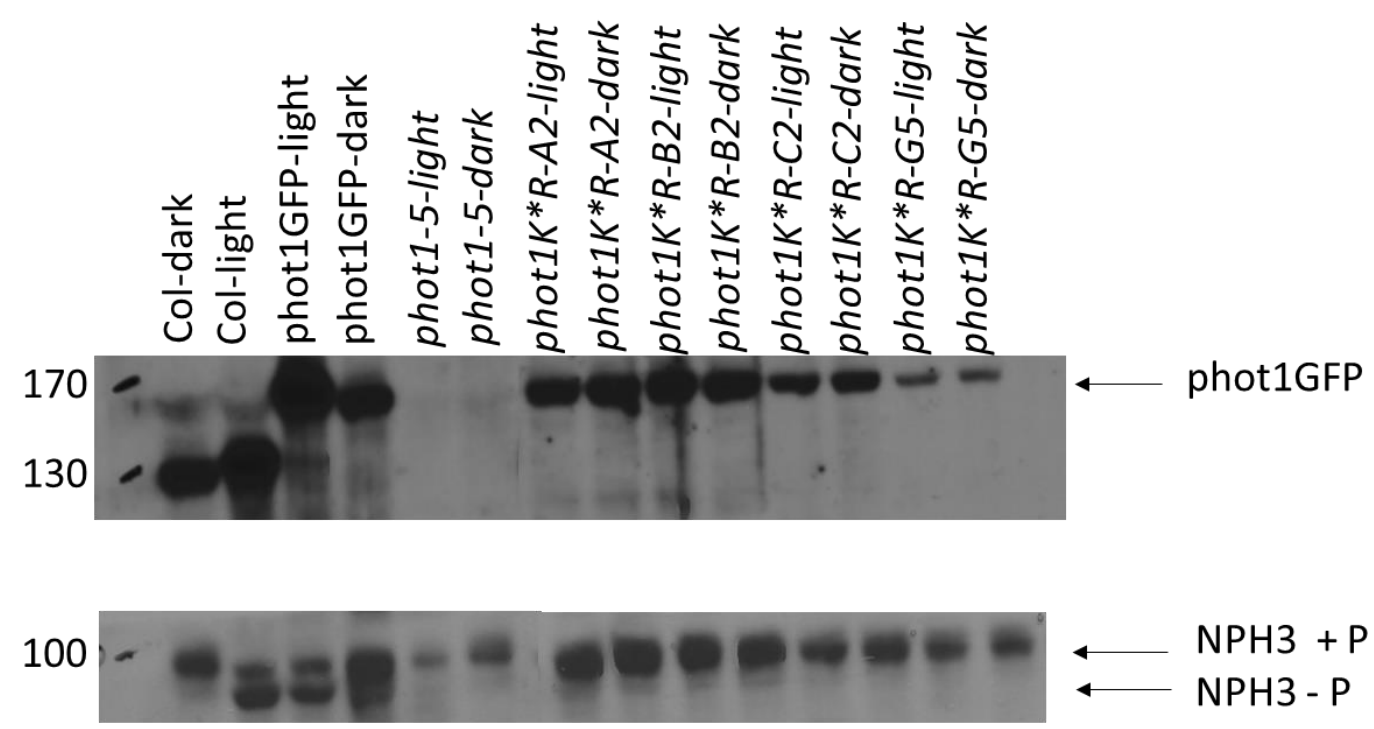

Figure 2-1 phot1 complete lysine-arginine mutant express normal levels of phot1 protein

3-day-old etiolated seedlings were exposed to saturating conditions of blue light (3000umol $\left.\mathrm{m}^{-2}\right)$ (Lascève et al., 1999). No phosphorylation band shift of phot 1 is detected in 4 independent phot $1 K^{*} R$ transgenic lines nor do we detect dephosphorylation of NPH3 in mutant seedlings. Phot1 total protein detected by anti-phot1 (Christie et al., 1998) dilution ratio 1:8000, NPH3 total protein detected by anti-NPH3 (Motchoulski and Liscum, 1999) dilution ratio 1:6000. 


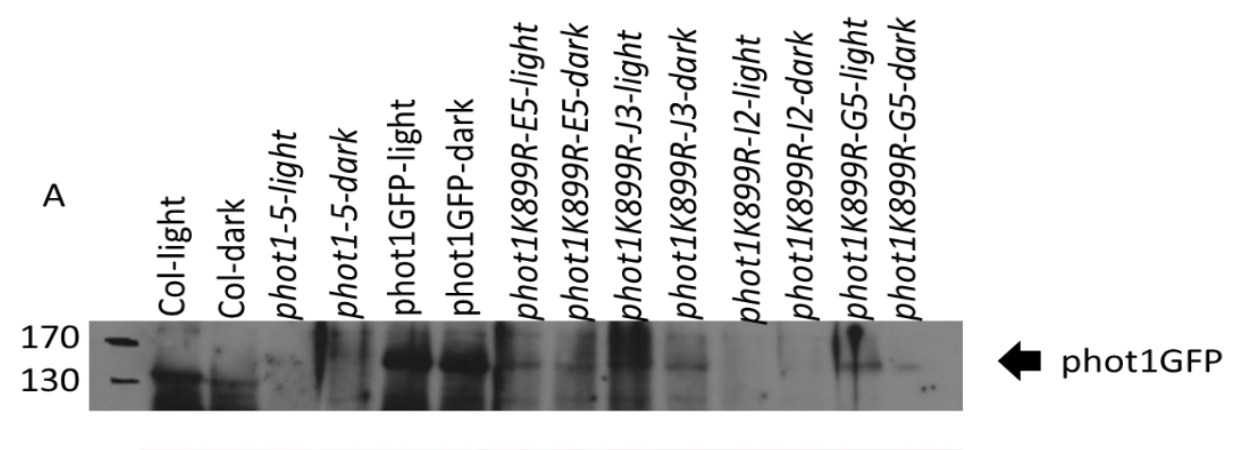

Ponceau
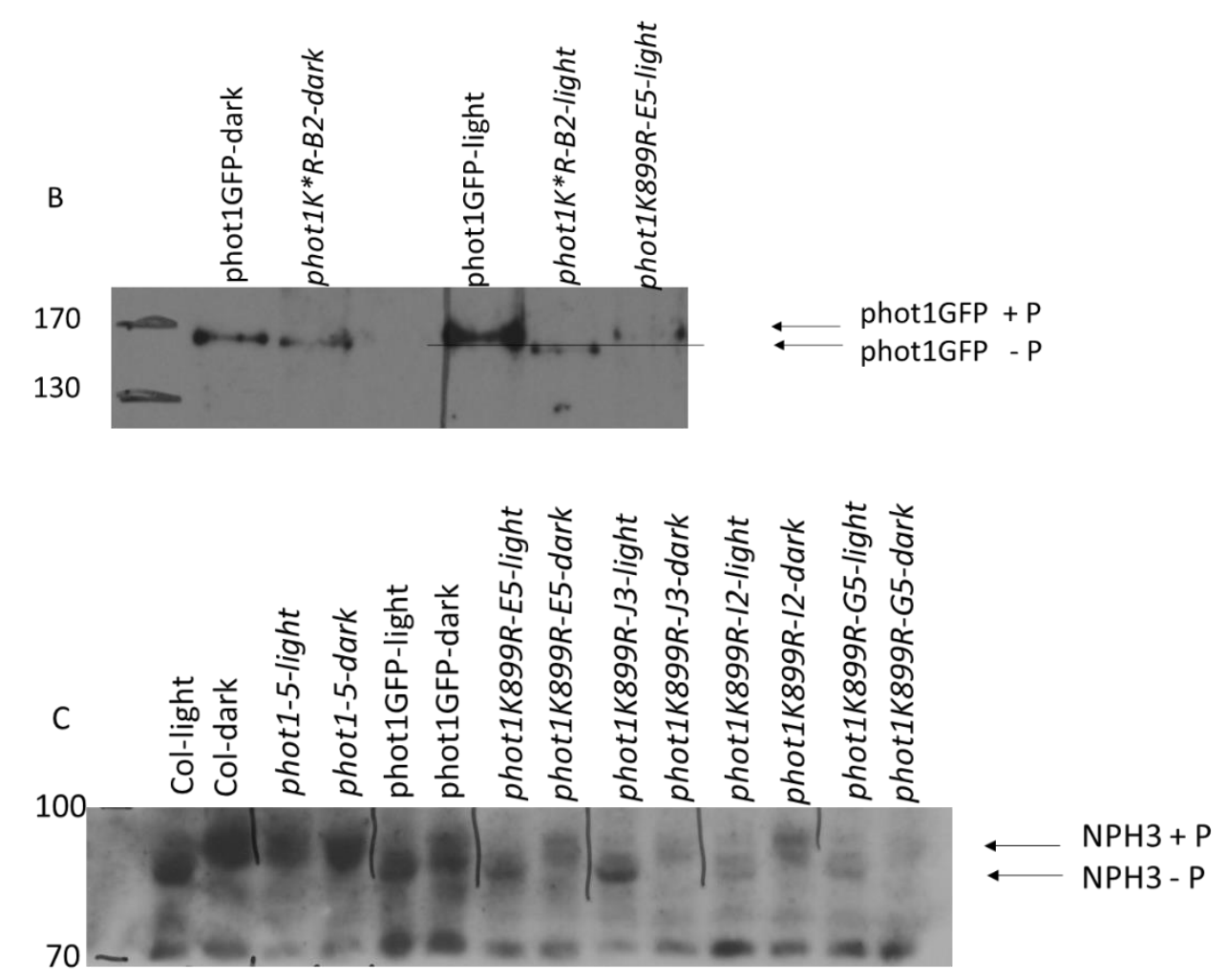

Figure 2-2 Protein expression in phot1K899R seedlings

A. 3-day-old etiolated seedlings were mock treated or exposed to saturating conditions of blue light (3000umol m²) (Lascève et al., 1999). Phot1 total protein detected by anti-phot1 (Christie et al., 1998) dilution ratio 1:8000. B. 3-day-old seedlings mock treated or exposed to 30 minutes blue light $\left(0.1 \mu \mathrm{mol} \mathrm{m} \mathrm{m}^{-2}\right.$ $\left.\mathrm{s}^{-1}\right) \cdot$ Immunoprecipitation by anti-GFP, protein detected by anti-phot1. Phosphorylation band shift is detected in phot1GFP and phot1K899R upon blue light exposure, but not in complete lysine mutant phot $1 K^{\star} R$. 
C. NPH3 total protein detected by anti-NPH3 (Motchoulski and Liscum, 1999) dilution ratio 1:6000. Normal dephosphorylation of NPH3 is detected in phot1K899R transgenic lines. 


\section{Phototropic Phenotypes of phot1 Ubiquitin Mutants}

Transgenic seedlings subjected to low fluence rate $\left(0.1 \mu \mathrm{mol} \mathrm{m}{ }^{-2} \mathrm{~s}^{-1}\right)$ unilateral blue light for 4 hours exhibited differing phototropic phenotypes based on mutation (Figure 2-3, 2-4 and 2-5). Phot1 complete lysine-arginine mutant $\left(K^{\star} R\right)$, while expressing normal levels of phot1 protein (Figure 2-1 top blot), do not exhibit a phototropic bending response upon blue light exposure (Figure 2-3). Knowing that autophosphorylation/transphosphorylation of the photoreceptor (Liscum and Briggs, 1995; Christie et al., 1998) are critical for phototropic signaling and dephosphorylation of NPH3 (Pedmale and Liscum, 2007), the observed lack of both of these responses in the $K^{\star} R$ (Figure 2-1, top and bottom blot) was not surprising given their lack of phototropic responsiveness.

Unlike what was observed with the $K^{\star} R$ lines, seedlings carrying the K899R mutation exhibit a quantitatively normal phototropic response (Figure 24). The normal phototropic bending response of phot1K899R is consistent with the observed autophosphorylation/transphosphorylation (Figure 2-2, A, B), and dephosphorylation of NPH3 (Figure 2-2, C) in these mutant seedlings. Since blue light and NPH3-dependent ubiquitination is necessary for phototropism (Roberts et al., 2011) and a normal phototropic response was observed, we predict that ubiquitination may still be occurring in the K899R lines, but at an alternate lysine.

Similar to phot1K899R findings, seedlings carrying the K526/527R substitutions also exhibited normal phototropic bending response under low intensity blue light (Figure 2-5). While ubiquitination at K526 was shown to be 
blue light-dependent (Deng et al., 2014), it appears not to be NPH3-dependent (Roberts, 2013) 


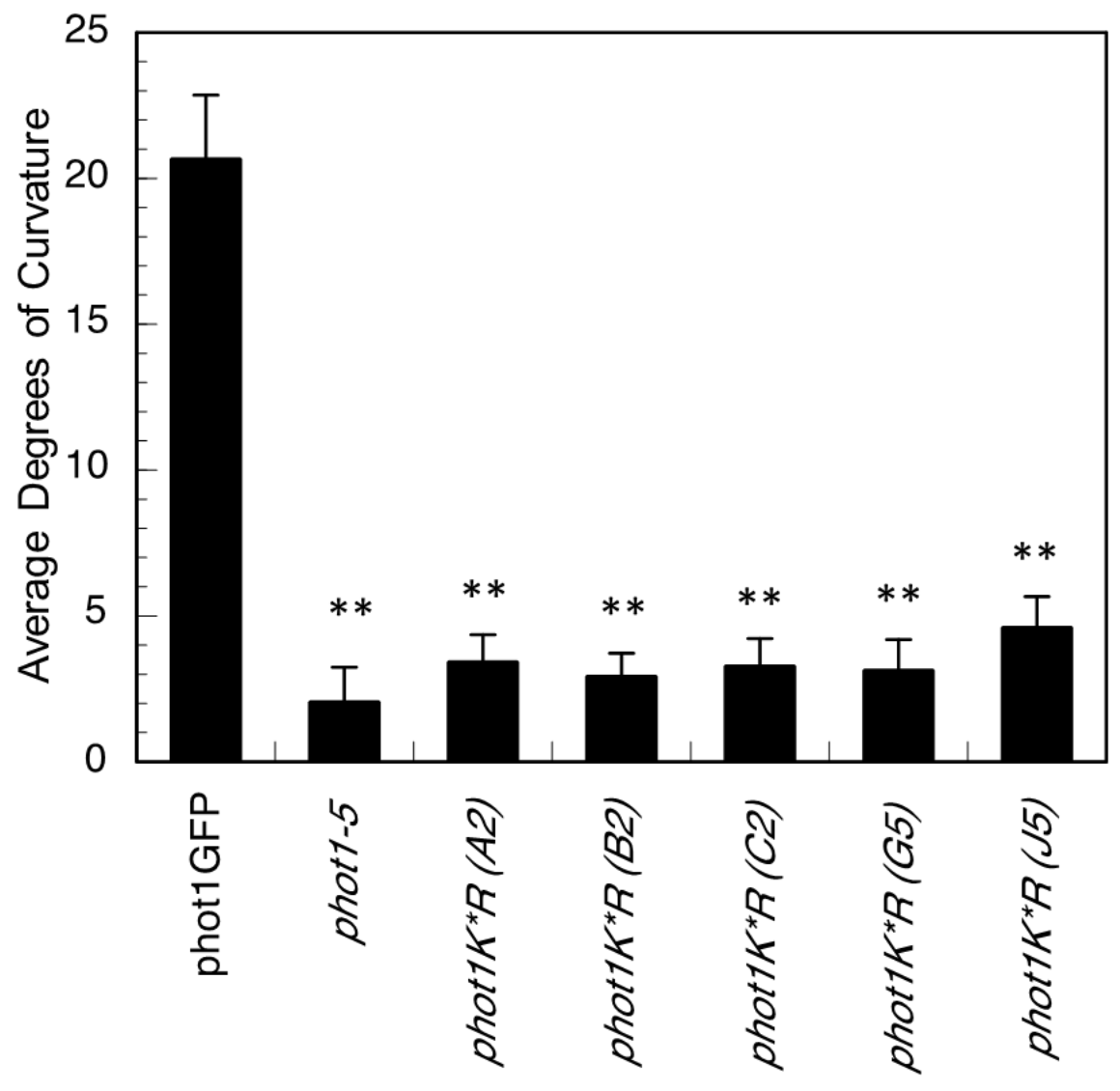

Figure 2-3 Hypocotyl phototropism of phot1 complete lysine mutant $\left(K^{*} R\right)$ seedlings

Hypocotyl phototropism of phot 1 transgenic lines for 4 hours of unilateral blue light at a fluence rate of 0.1 $\mu \mathrm{mol} \mathrm{m} \mathrm{m}^{-2} \mathrm{~s}^{-1}$. Positive control represented by phot1GFP (Sakamoto and Briggs, 2002) and the null allele phot $1-5$ represents the negative control. Data is represented by average degrees of curvature with standard error denoted from three independent replicate experiments ( $n>68$ seedlings). Statistical significance determined by a students T-test, of mean average differing from that of phot1GFP is denoted with two asterisks $(\mathrm{P}<0.01)$. 


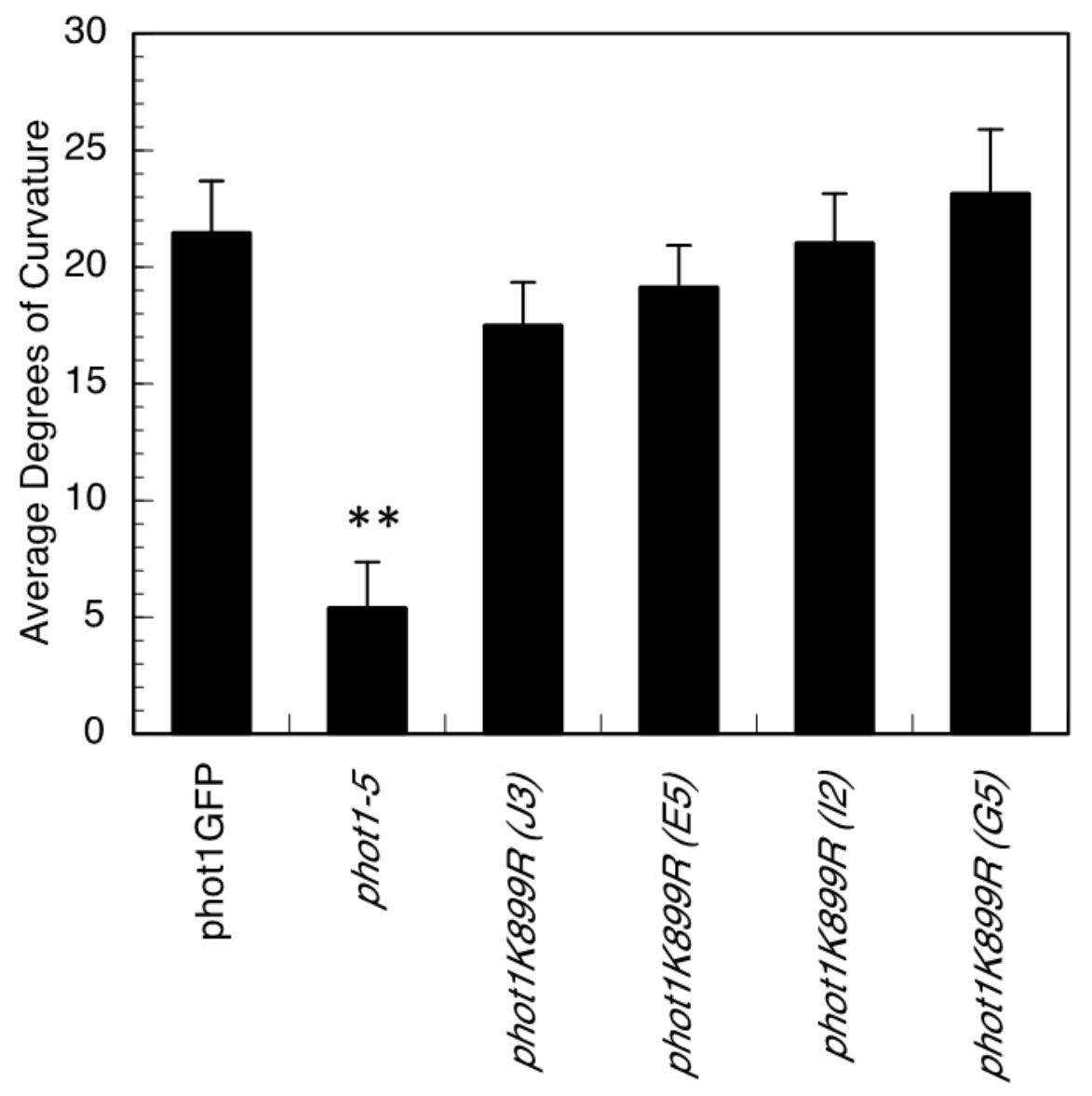

Figure 2-4 Hypocotyl phototropism in phot1K899R ubiquitin mutant seedlings

Hypocotyl phototropism of phot 1 transgenic lines for 4 hours of unilateral blue light at a fluence rate of 0.1 $\mu \mathrm{mol} \mathrm{m} \mathrm{m}^{-2} \mathrm{~s}^{-1}$. Positive control represented by phot1GFP (Sakamoto and Briggs, 2002)and the null allele phot $1-5$ represents the negative control. Data is represented by average degrees of curvature with standard error denoted from three independent replicate experiments ( $n>50$ seedlings). Statistical significance determined by a students T-test, of mean average differing from that of phot1GFP is denoted with two asterisks $(\mathrm{P}<0.01)$. 


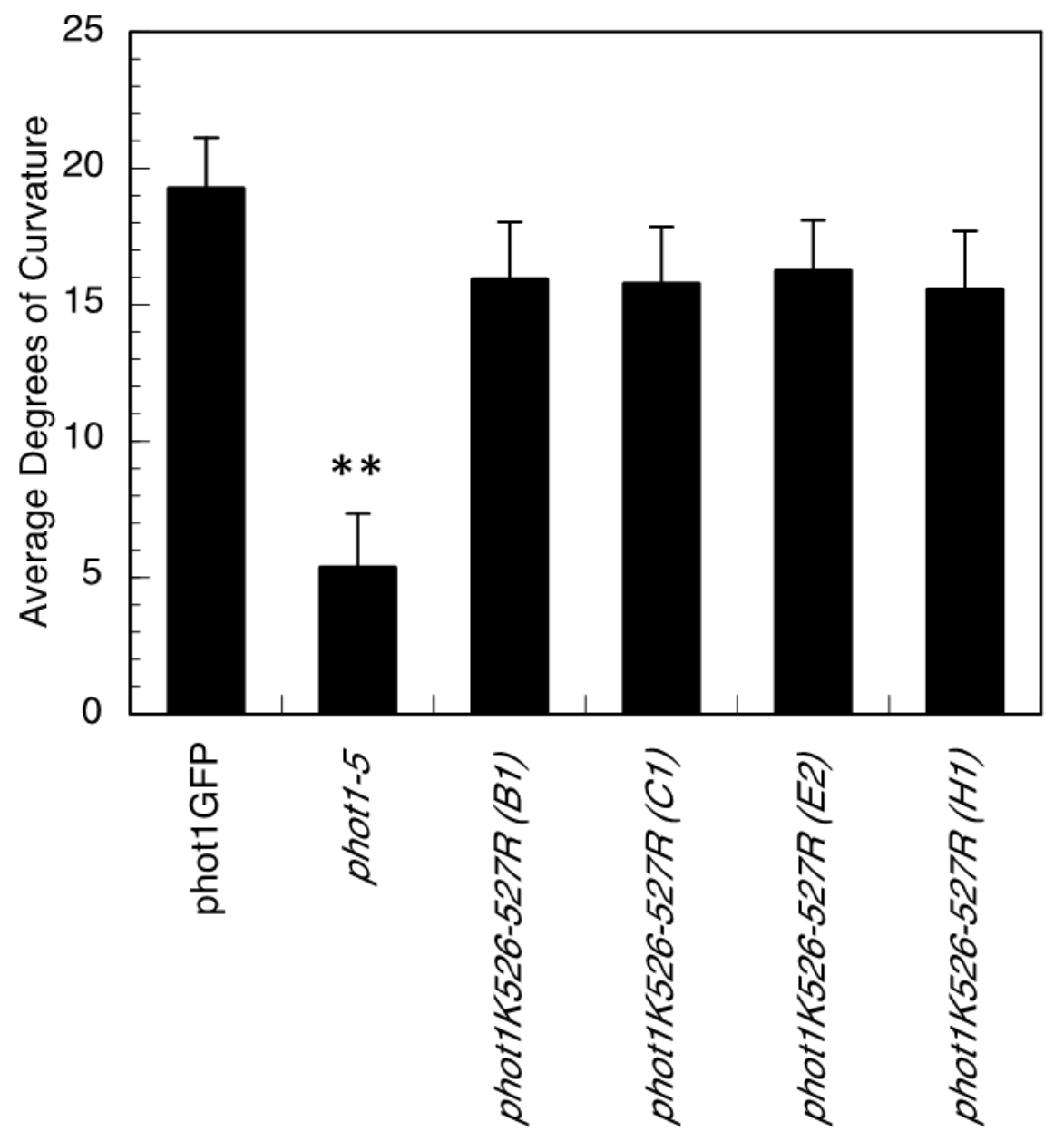

Figure 2-5 Hypocotyl phototropism of phot1K526-527R ubiquitin mutant seedlings

Hypocotyl phototropism of phot 1 transgenic lines for 4 hours of unilateral blue light at a fluence rate of 0.1 $\mu \mathrm{mol} \mathrm{m} \mathrm{m}^{-2} \mathrm{~s}^{-1}$. Positive control represented by phot1GFP (Sakamoto and Briggs, 2002) and the null allele phot $1-5$ represents the negative control. Data is represented by average degrees of curvature with standard error denoted from three independent replicate experiments ( $n>66$ seedlings). Statistical significance determined by a students T-test, of mean average differing from that of phot1GFP is denoted with two asterisks $(\mathrm{P}<0.01)$. 


\section{Leaf Movement Response is Normal in phot1K899R Seedlings}

As previously mentioned, phot1 mediates a number of growth responses (Christie et al., 2015). Of these responses, NPH3 has been shown to be critical only for the phototropic bending response (Liscum and Briggs, 1996) and leaf movement response (Inada et al., 2004; Inoue et al., 2008b). We examined petiole angles in seedlings carrying this substitution to characterize a second phot1-mediated function in which NPH3 is involved. Angles were measured from photographed images of seedlings. Angle vertex is where the petiole is attached to the hypocotyl, with one ray forming a horizontal line at this attachment and the second moving up along the petiole (Figure 2-6). Maybe not surprising, given the normal phototropic responsiveness of the K899R lines, we also observed normal leaf movement in three transgenic lines, however, we do see a significantly different phenotype in one line (phot1K899R-J3) (Figure 2-6). More replicates of this line may need to be performed to further examine this phenotype. However, as we do not see a statistically significant change in leaf movement in other transgenic lines, this leads to the conclusion that ubiquitination may be occurring in phot1K899R seedlings at an alternate lysine, or perhaps that phot1 ubiquitination is not necessary for this response. 
A.

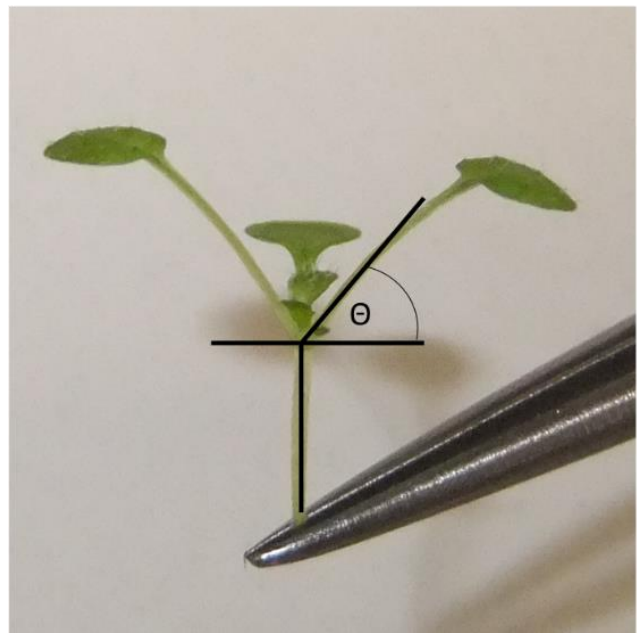

B.

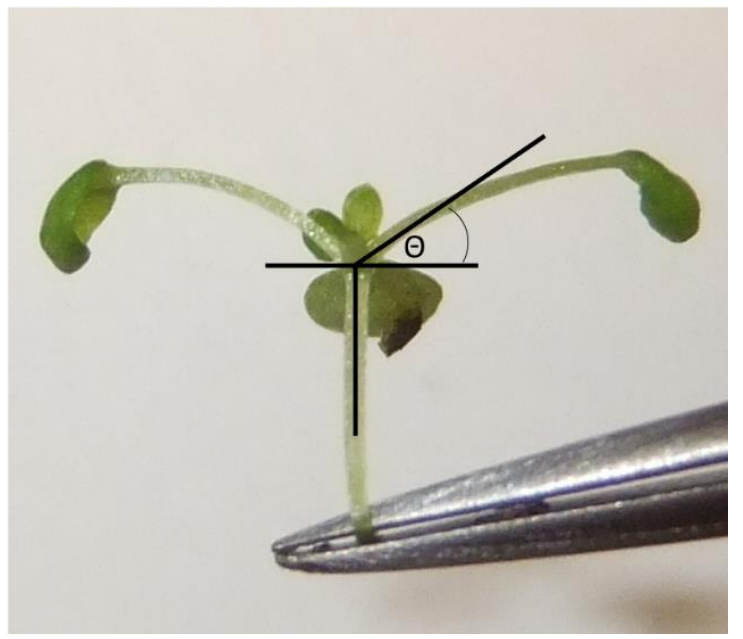

Figure 2-6 Petiole angle measurement example

Pictures were taken of 5-day-old seedlings grown in red $\left(25 \mu \mathrm{mol} \mathrm{m} \mathrm{m}^{-2} \mathrm{~s}^{-1}\right)$ plus blue light $\left(0.2 \mu \mathrm{mol} \mathrm{m}^{-2} \mathrm{~s}^{-1}\right)$. Images were measured using FIJI software. $\Theta$ represents angle measured. A. Columbia, wildtype seedling, B. NPH3 null mutant: nph3-6 seedling. 


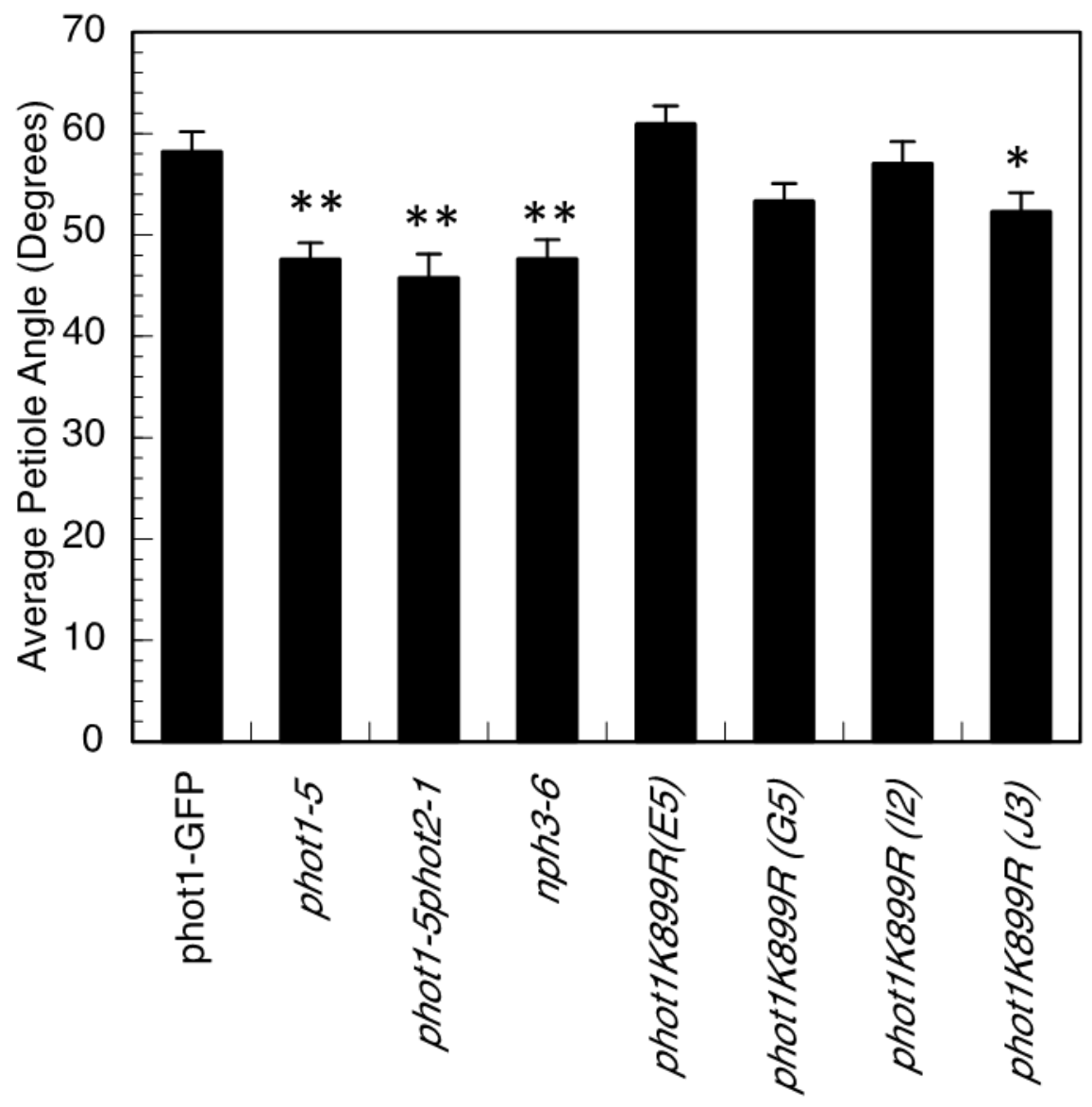

Figure 2-7 Leaf movement observed in phot1K899R mutant seedlings

Leaf movement of phot 1 transgenic lines is identified by measuring petiole angle of seedlings in red $(25$ $\left.\mu \mathrm{mol} \mathrm{m} \mathrm{m}^{-2} \mathrm{~s}^{-1}\right)$ plus blue light $\left(0.2 \mu \mathrm{mol} \mathrm{m} \mathrm{m}^{-2} \mathrm{~s}^{-1}\right)$ for 5 days. Positive control represented by phot1GFP (Sakamoto and Briggs, 2002) and the null alleles phot1-5, nph3-6 as well as a phototropin double mutant (phot1-5phot2-1) represents the negative control. Data is represented by average petiole angle in degrees with standard error denoted from three independent replicate experiments ( $n>20$ seedlings). Statistical significance determined by a students T-test, of mean average differing from that of phot1GFP is denoted with an asterisk $(P<0.05)$, or two asterisks $(P<0.01)$. 


\section{Characterization of Another phot1-Mediated Function Which Does Not Involve NPH3}

Chloroplast movement is a response that is mediated by both members of the phototropin family of photoreceptors: both phot 1 and phot2 mediate chloroplast accumulation, while phot2 alone mediates chloroplast avoidance (Jarillo et al., 2001; Kagawa et al., 2001). In darkness, chloroplasts are randomly distributed within individual cells, but upon exposure to low intensity blue light, chloroplasts exhibit the accumulation response in which they will move to the periclinal sides of the cell to maximize their photosynthetic light capture.

However, in high intensity blue light, chloroplasts move and stack along the anticlinal sides of the cell in order to avoid photodamage (Figure 2-8) (Kong and Wada, 2016). While NPH3 is not involved in this response, two other members of the NRL protein family, RPT2 and NRL31/NCH1/SR1IP1 have been shown to be involved (Suetsugu et al., 2016).

When we examined the chloroplast movement responses in our phot ubiquitin mutant lines, we found that the $K^{\star} R$ lines exhibited responses similar to that of the phot1-5 null-mutant (Figure 2-9). These phenotypes were not surprising given that lack of early biochemical responses (Figure 2-1) and phototropic bending response (Figure 2-3). Surprisingly, given their normal biochemical (Figure 2-2), phototropic (Figure 2-4), and leaf angle (Figure 2-7) responses, K899R lines also exhibited a phenotype more similar to that of the phot1-5 null mutant than that of the positive phot1GFP control (Figure 2-10). While we observe a loss-of-function in $K^{*} R$ and K899R lines compared to the 
positive control in chloroplast accumulation, we do not see a loss-of-function in these lines in the chloroplast avoidance response.

As previously mentioned, the NRL member NRL31/NCH1/SR1IP1 is involved in chloroplast accumulation, and has been shown to interact with phot1 similarly to NPH3, through its CC domain (Suetsugu et al., 2016). Zhang and colleagues (2014) have shown that NRL31/NCH1/SR1IP1 can also interact with CUL3a through its BTB domain and act as a substrate adapter in an E3 ubiquitin ligase complex involved in plant pathogen responses. Based on these findings we speculate that perhaps ubiquitination at K899 is necessary for chloroplast accumulation in a non-NPH3-dependent manner. Conceivably NRL31/NCH1/SR1IP1, rather than NPH3, may act as a substrate adapter in a CRL3 ubiquitin ligase complex in the chloroplast accumulation response.

We observed a variable response in chloroplast accumulation in lines carrying the phot1K526/527R substitution (Figure 2-11). As previously mentioned K526 was identified as a blue light dependent ubiquitination site (Deng et al., 2014), that is not NPH 3-depdendent (Roberts, 2013). While we do observe normal phototropic responsiveness in these lines, it is critical to identify phot 1 protein expression in individual lines, as perhaps this may explain the variable chloroplast accumulation phenotypes. 


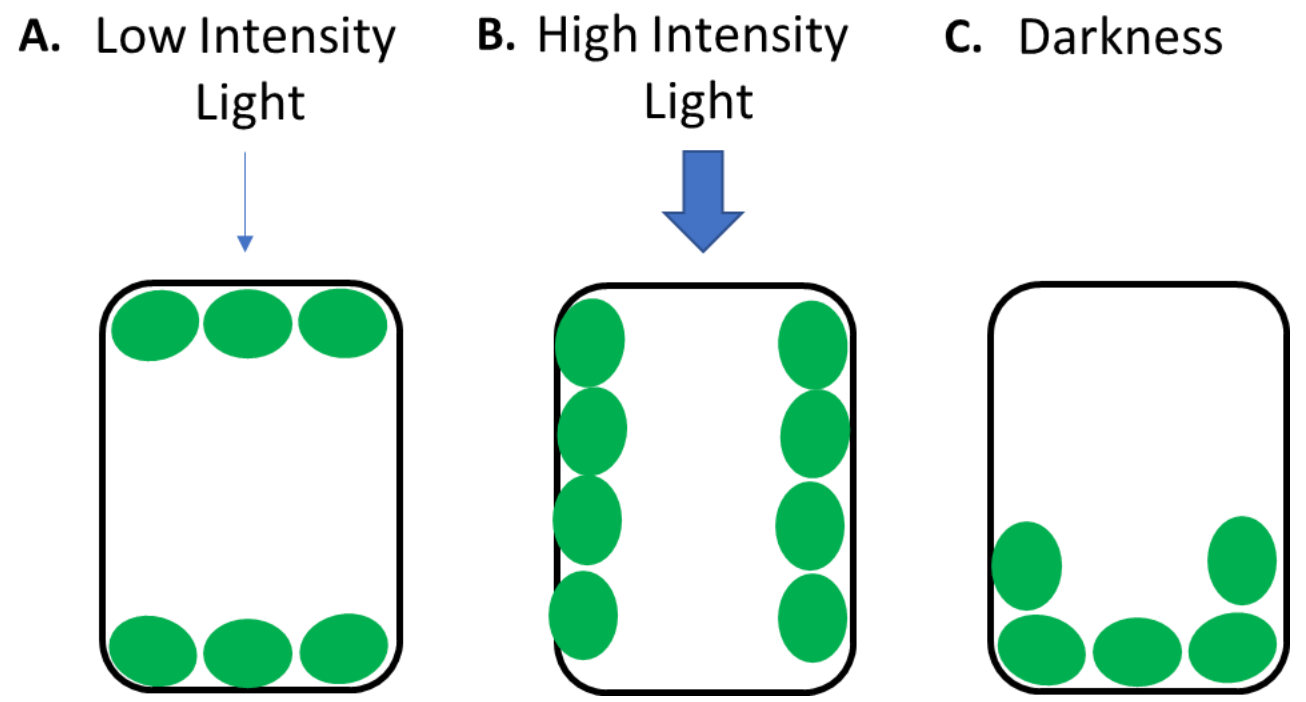

Figure 2-8 Chloroplast movement upon differential light intensity exposure

Schematic diagram of chloroplasts location within an individual cell in A. low intensity light, B. high intensity light and C. darkness. Image from S. G. Kong \& Wada, 2016. 


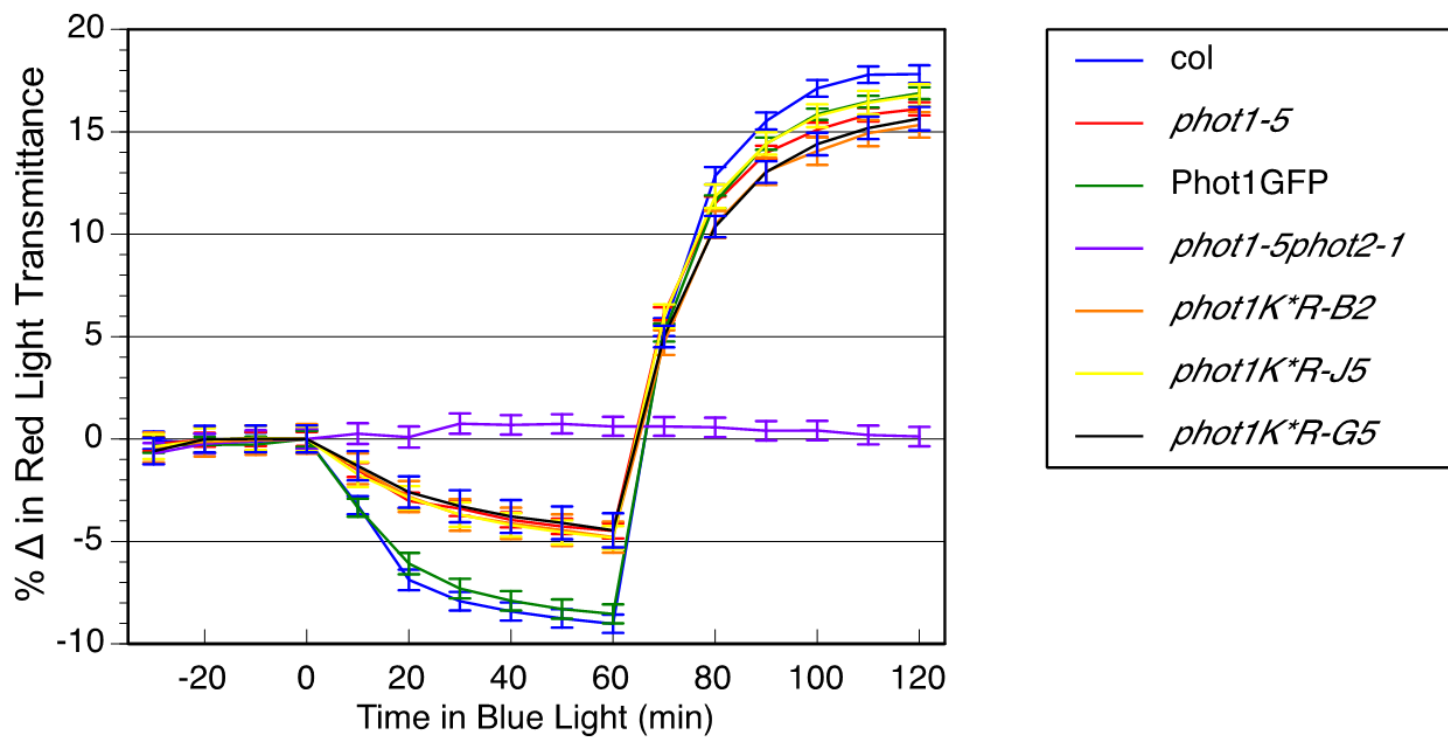

Figure 2-9 Chloroplast movement in phot1 complete lysine mutant seedlings

3-5-week-old rosette leaf discs were dark adapted, then exposed to low intensity light $\left(0.1 \mu \mathrm{mol} \mathrm{m}^{-2} \mathrm{~s}^{-1}\right)$ for 60 minutes, and then exposed to high intensity light $\left(75 \mu \mathrm{mol} \mathrm{m}^{-2} \mathrm{~s}^{-1}\right)$ for 60 minutes. Measurements were taken every 10 minutes to measure the amount of red light transmitted through leaf discs. Data from three replicate experiments is plotted by percent change in red light transmittance compared to the 0 -time point of each line. 


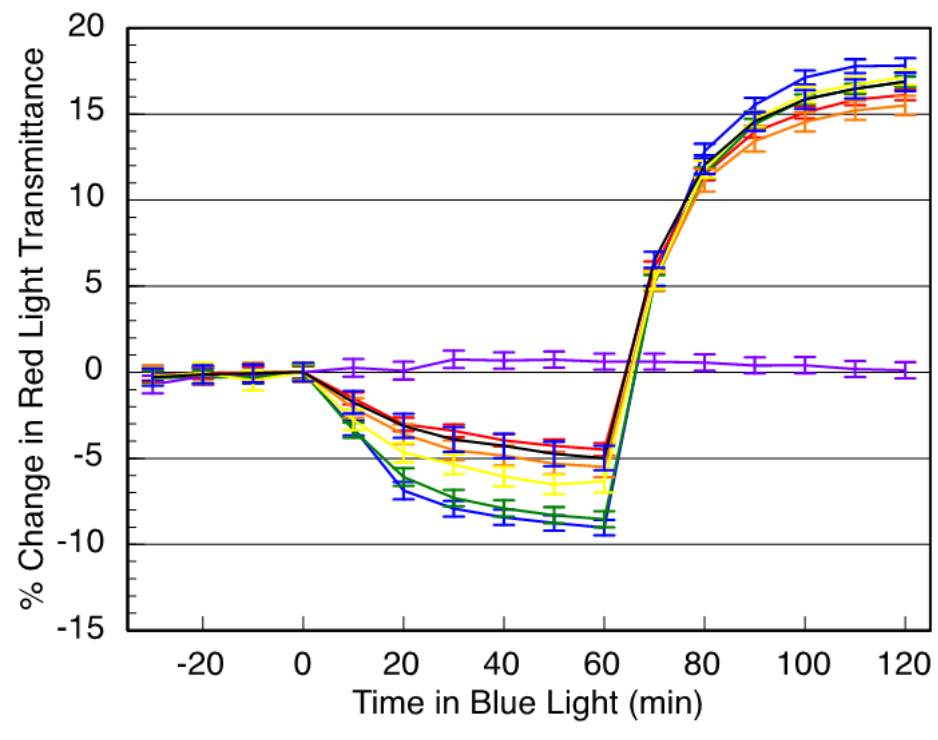

\begin{aligned} \hline- & col \\ - & phot1-5 \\ - & Phot1GFP \\ - & phot1-5phot2-1 \\ - & phot1k899R (E5) \\ & phot1K899R (G5) \\ - & phot1K899R (I2) \end{aligned}

Figure 2-10 Chloroplast movement in phot1K899R mutant seedlings

3-5-week-old rosette leaf discs were dark adapted, then exposed to low intensity light $\left(0.1 \mu \mathrm{mol} \mathrm{m} \mathrm{m}^{-2} \mathrm{~s}^{-1}\right)$ for 60 minutes, and then exposed to high intensity light $\left(75 \mu \mathrm{mol} \mathrm{m}^{-2} \mathrm{~s}^{-1}\right)$ for 60 minutes. Measurements were taken every 10 minutes to measure the amount of red light transmitted through leaf discs. Data from three replicate experiments is plotted by percent change in red light transmittance compared to the 0 -time point of each line. 


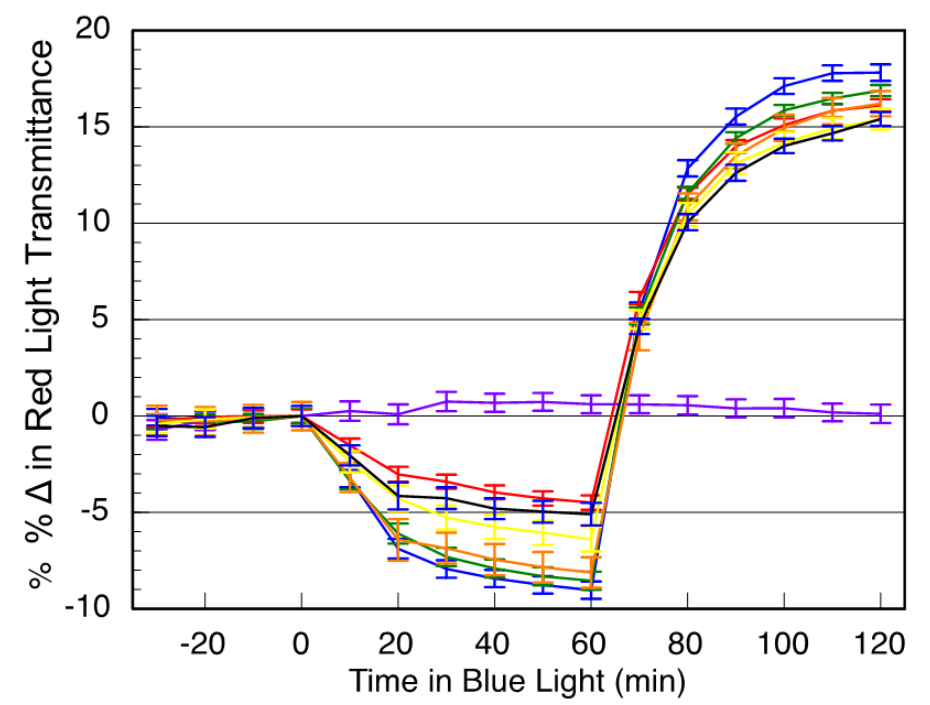

$$
\begin{aligned}
- & \text { col } \\
- & \text { phot1-5 } \\
- & \text { phot1GFP } \\
- & \text { phot1-5phot2-1 } \\
- & \text { phot1K526-527R-B1 } \\
& \text { phot1K526-527R-E2 } \\
- & \text { phot1K526-527R-H1 }
\end{aligned}
$$

Figure 2-11 Chloroplast movement in phot1K526-527R mutant seedlings

3-5-week-old rosette leaf discs were dark adapted, then exposed to low intensity light $\left(0.1 \mu \mathrm{mol} \mathrm{m}^{-2} \mathrm{~s}^{-1}\right)$ for 60 minutes, and then exposed to high intensity light $\left(75 \mu \mathrm{mol} \mathrm{m}^{-2} \mathrm{~s}^{-1}\right)$ for 60 minutes. Measurements were taken every 10 minutes to measure the amount of red light transmitted through leaf discs. Data from three replicate experiments is plotted by percent change in red light transmittance compared to the 0 -time point of each line. 


\section{Characterization of phot1 Ubiquitination in Mutant Plants}

As previously reported by Roberts and colleagues (2011), phot1

polyubiquitination under high blue light conditions was not detected without the use of a protease inhibitor to stabilize the protein. Therefore, we pretreated phot1 ubiquitination mutant lines with DMSO (mock treatment) or with MG132 (a 26S proteasome inhibitor), two hours prior to a time course high blue light treatment. Similar to the findings of Roberts and colleagues (2011), total protein immunoblots show phot1 protein levels stabilized by MG132 pretreatment as compared to mock treatment in control seedlings as well as phot1 ubiquitination mutants (K899R and $K^{\star} R$ ) in both low and high intensity blue light conditions (Figure 2-13, 2-14). 

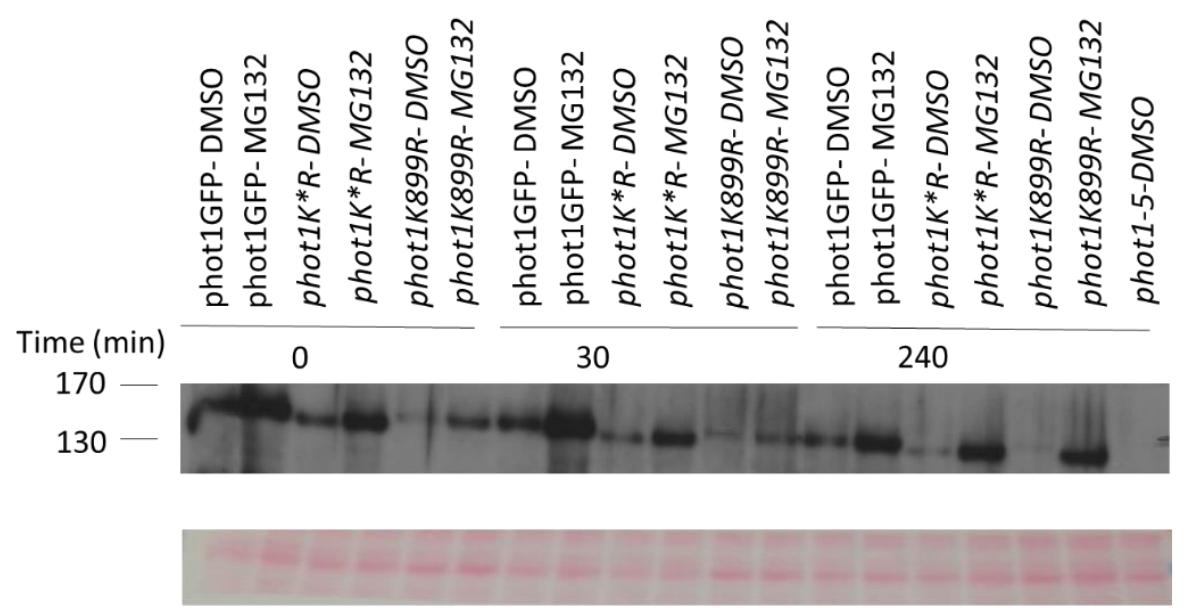

Ponceau

Figure 2-12 Total protein of mock or MG132 treated low light time course seedlings

3-day-old etiolated seedlings were treated with DMSO (mock) or MG132 2 hours prior to a time course light treatment. Seedlings were exposed to low intensity blue light $\left(0.1 \mu \mathrm{mol} \mathrm{m}{ }^{-2} \mathrm{~s}^{-1}\right)$ and collected at 0,30 and 240-minute time points. Phot1 total protein detected by anti-phot1 (Christie et al., 1998) dilution ratio 1:8000. 


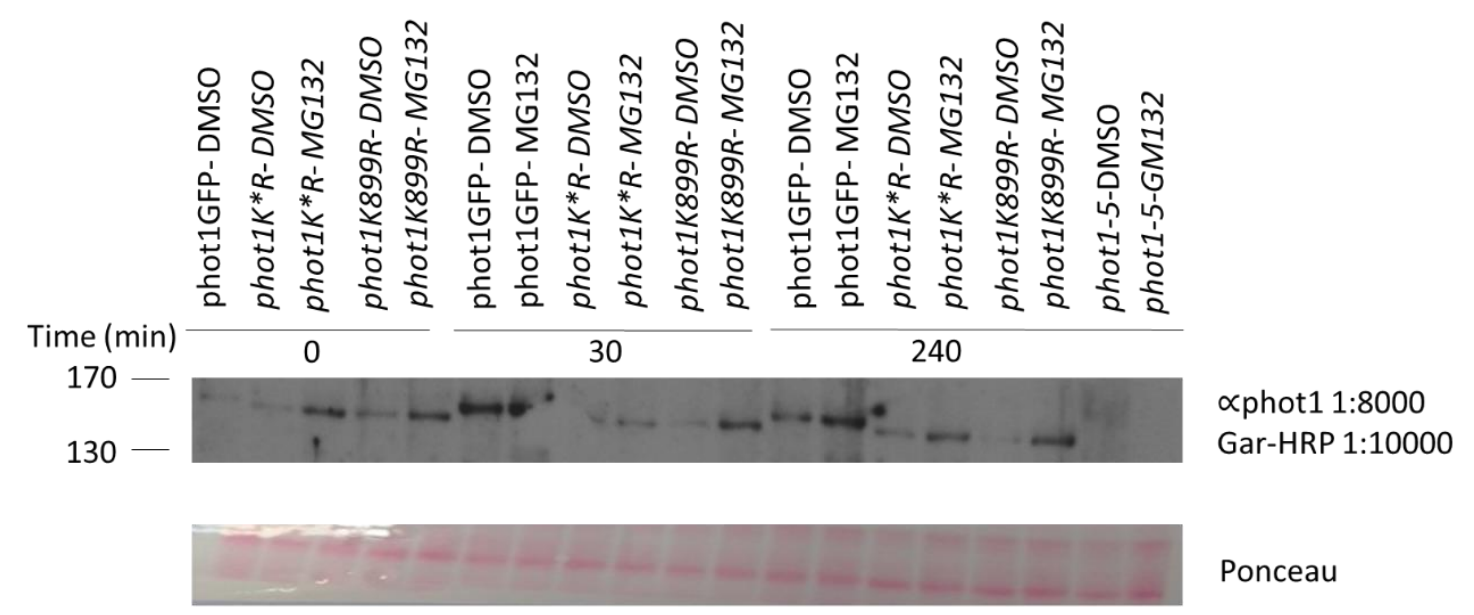

Figure 2-13 Total protein of mock or MG132 treated high light time course seedlings

3-day-old etiolated seedlings were treated with DMSO (mock) or MG132 2 hours prior to a time course light treatment. Seedlings were exposed to high intensity blue light $\left(117 \mu \mathrm{mol} \mathrm{m}^{-2} \mathrm{~s}^{-1}\right)$ and collected at 0,30 and 240-minute time points. Phot1 total protein detected by anti-phot1 (Christie et al., 1998) dilution ratio 1:8000. 
To assess phot1 ubiquitination in mutant plants we utilized the GFP protein (which is translationally-fused to our phot1 control and mutant proteins) to immunoprecipitate phot1 protein. After immunoprecipitation, we performed immunoblot analysis using native phot1 antibody and a ubiquitin-specific antibody which recognizes both monoubiquitination and polyubiquitination. Multiple replicates have confirmed that indeed both the phot 1 complete lysinearginine substitution $\left(K^{\star} R\right)$, as well as the critical K899R mutant are both ubiquitinated under high and low intensity light conditions (Figure 2-15, 2-16). In fact, there does not appear to be less ubiquitin detected in our ubiquitin mutant transgenic seedlings. There can be multiple explanations for our lack of ubiquitination change within our mutant seedlings. It's been long shown that ubiquitination is promiscuous. If a native ubiquitination site is unavailable to the ubiquitination machinery, it's been shown that ubiquitin can be covalently linked to a nearby lysine within the protein (Stringer and Piper, 2011). Additionally, it's been shown that if no lysines are present, protein degradation marked by the polyubiquitination can still occur (Gilkerson et al., 2015). 


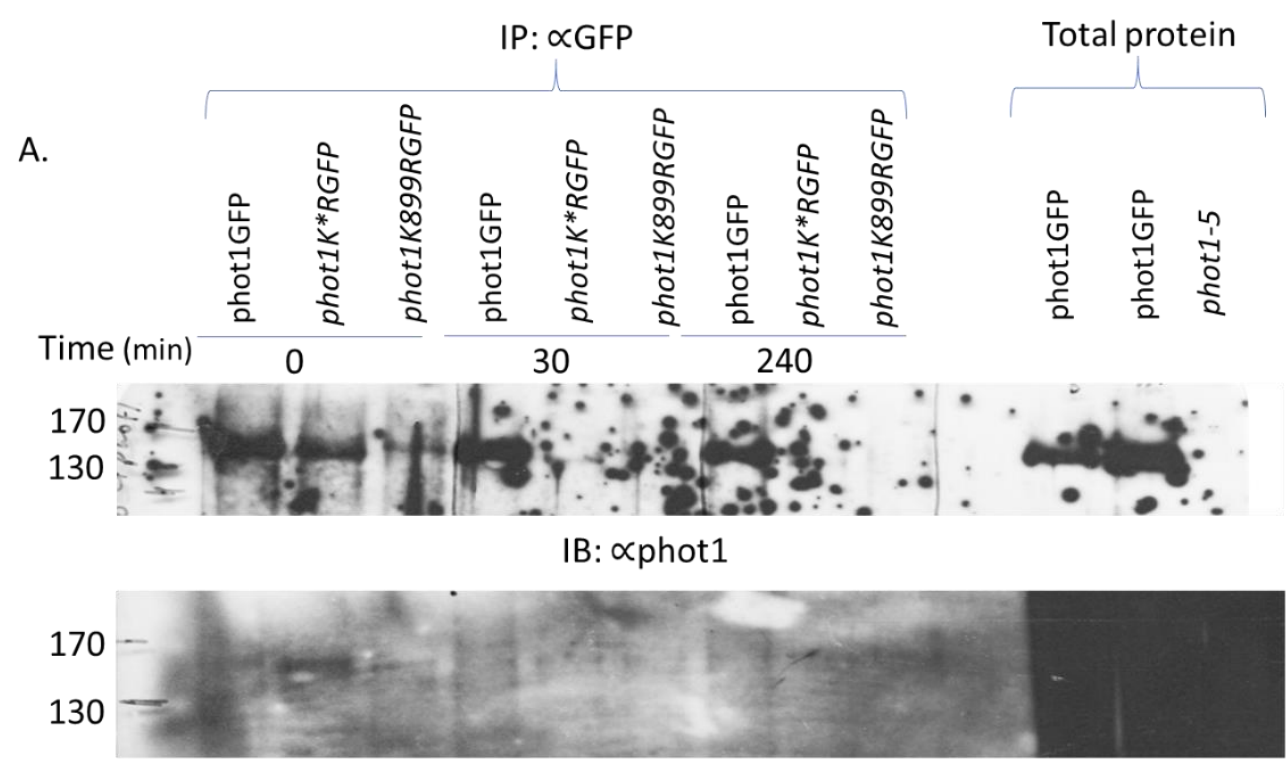

IB: $\propto$ UB

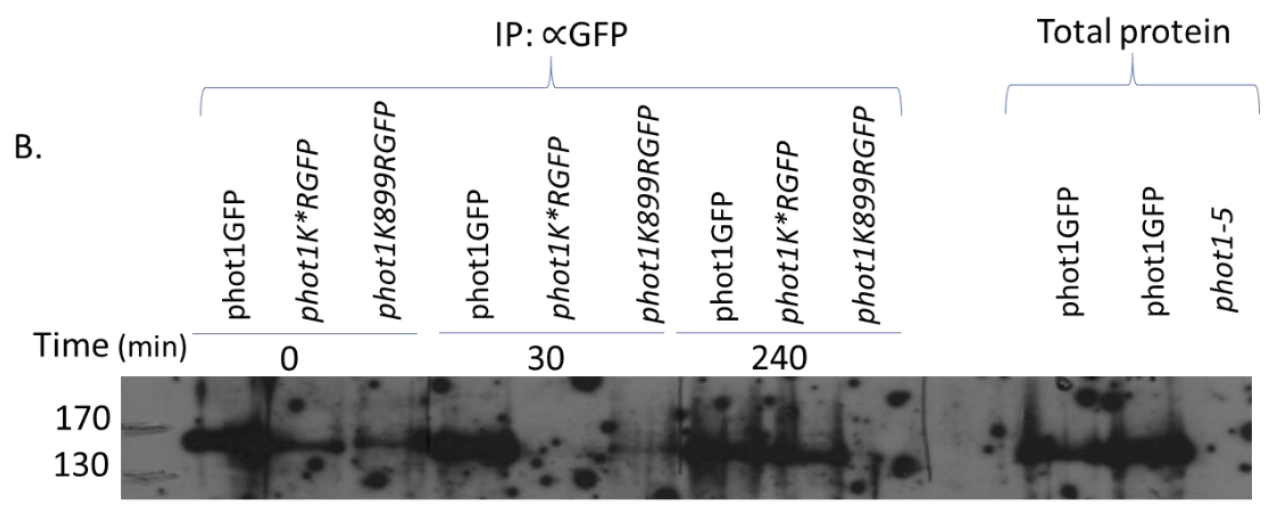

IB: $\propto$ phot1

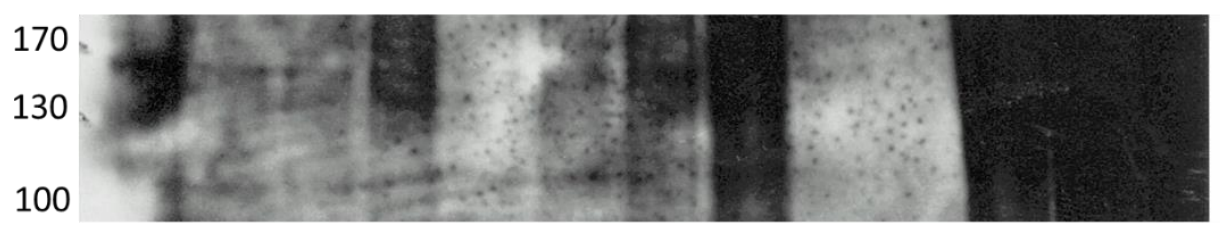

IB: $\propto U B$

Figure 2-14 Low light phot1 ubiquitination

3-day-old etiolated seedlings were treated with (A) DMSO (mock) or (B) MG132 2 hours prior to a time course light treatment. Seedlings were exposed to low intensity blue light $\left(0.1 \mu \mathrm{mol} \mathrm{m} \mathrm{m}^{-2} \mathrm{~s}^{-1}\right)$ and collected at 0, 30 and 240-minute time points. Phot1 protein was immunoprecipitated by anti-GFP and 
immunoprecipitated phot1 protein was detected by anti-phot1 (Christie et al., 1998) dilution ratio 1:8000 and ubiquitin detected by anti-P4D1 (Cell Signaling, 3936S) dilution ratio 1:1500. 


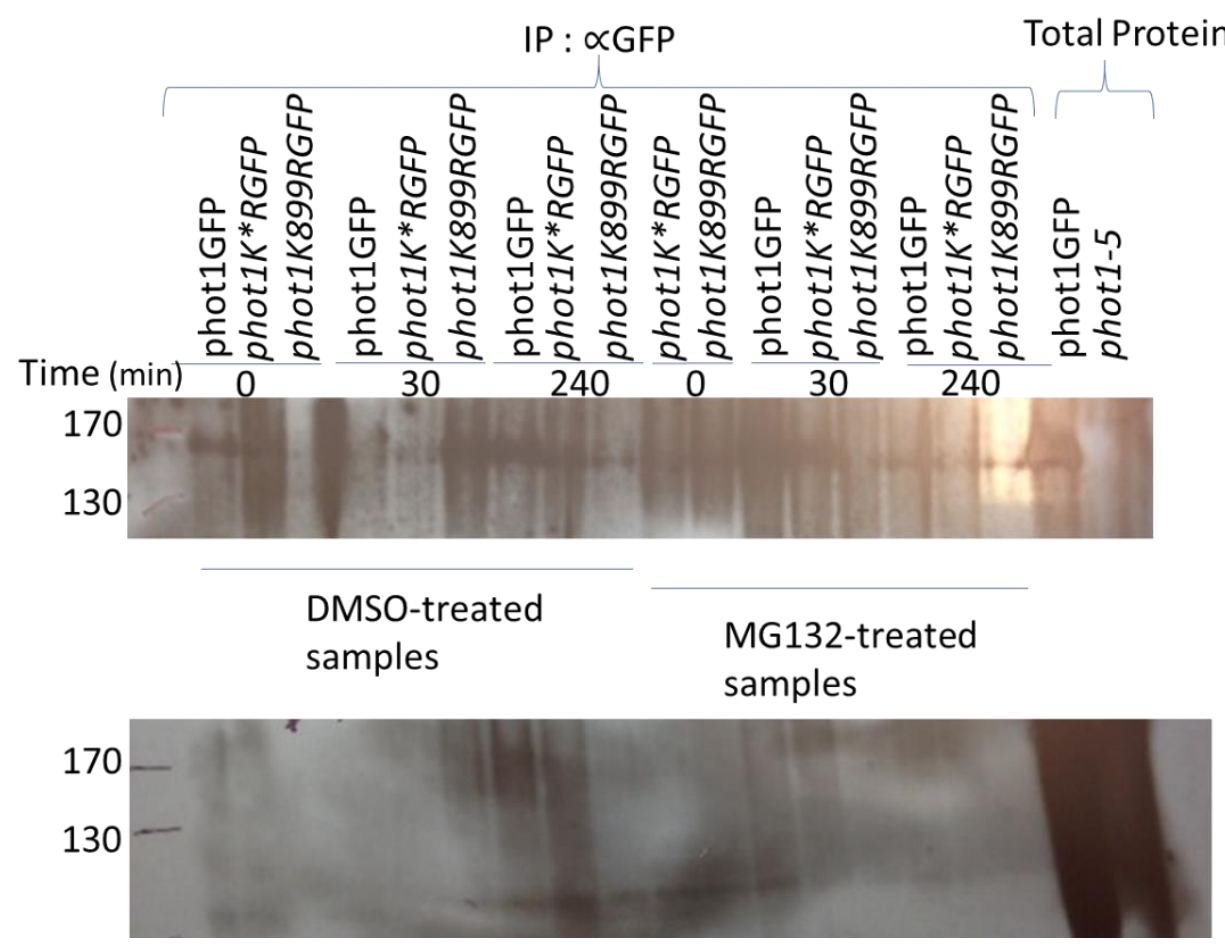

IB: $\propto$ phot1

Figure 2-15 High light phtot1 ubiquitination

3-day-old etiolated seedlings were treated with DMSO (mock) or MG132 2 hours prior to a time course light treatment. Seedlings were exposed to high intensity blue light $\left(117 \mu \mathrm{mol} \mathrm{m}^{-2} \mathrm{~s}^{-1}\right)$ and collected at 0,30 and 240-minute time points. Phot1 protein was immunoprecipitated by anti-GFP and immunoprecipitated phot1 protein was detected by anti-phot1 (Christie et al., 1998) dilution ratio 1:8000 and ubiquitin detected by antiP4D1 (Cell Signaling, 3936S). 


\section{Discussion}

Ubiquitination has been shown to be involved in a variety of cellular functions such as endocytosis, nuclear transport and protein degradation, among others (Haglund and Dikic, 2005). phot1 has been shown to be ubiquitinated upon blue light exposure in an NPH3-dependent fashion (Roberts et al., 2011). This ubiquitination leads to varying outcomes dependent on light intensity. For example, under high intensity blue light, phot1 is polyubiquitinated leading to receptor degradation by the $26 \mathrm{~S}$ proteasome. In contrast, under low intensity blue light phot1 is monoubiquitinated rather than polyubiquitinated (Roberts et al., 2011). The function of phot1 monoubiquitination has recently been suggested as means to retain phot1 signaling at the plasma membrane (Askinosie, 2016). Under high intensity blue light, phototropic bending may not be necessary as the plant is receiving enough light to perform photosynthesis; however, under low intensity blue light it is more imperative that the plant maximize its photosynthetic light capture.

We assessed several phot1-mediated functions in plants containing a mutation at the previously identified blue light- and NPH3-dependent ubiquitination site (K899R) (Roberts et al., 2011), as well as a previously identified blue light-dependent, NPH3-independent ubiquitination site (K526/527) (Deng et al., 2014). In addition to these phot1 ubiquitin mutants, we assessed plants containing a mutant phot1 protein where all lysine residues were mutated to arginine in an effort to prevent ubiquitination from occurring $\left(K^{\star} R\right)$. 
Plants with all ubiquitination sites mutated express phot1 protein, but exhibit a non-phototropic phenotype, similar to the phot1-5 null mutant (Figure 21). These results are not surprising as we did not observe phosphorylation of phot1 nor dephosphorylation of NPH3, both of which have been shown to be necessary for this response (Liscum and Briggs, 1995; Pedmale and Liscum, 2007). Additionally, these $K^{\star} R$ plants exhibited a chloroplast accumulation, similar to the phot 1-5 mutant. As 80 amino acids within this protein have been substituted from lysine to arginine, it is not hard to image that the general structure of this protein may have been altered in a way in which affects this proteins function. It would be interesting to ascertain in the future whether or not this protein is perceiving light. Interestingly, in the absence of lysines within the phot1 protein, we still detect ubiquitin modification on phot1 (Figure 2-14, 2-15). Gilkerson and colleagues (2015) found that proteins with lysine substitutions can still be ubiquitinated at serine/threonine through the hydroxyl group. It has additionally been reported that ubiquitin can alternatively bind at a cysteine as well as at the amino-terminus of a protein (McDowell and Philpott, 2013).

Contrary to the phot 1 complete lysine-arginine substitution $\left(K^{\star} R\right)$, the single phot ubiquitin mutant (K899R) exhibits normal phototropic curvature (Figure 2-4) as well normal leaf movement (Figure 2-7) with the exception of one transgenic line. Both of these phot1-mediated functions involve the substrate adapter NPH3 (Liscum and Briggs, 1996; Inoue et al., 2008b; Roberts et al., 2011), and lysine899 was identified as the blue light- and NPH3-dependent ubiquitination of phot1 (Roberts, 2013). As ubiquitination can be promiscuous, 
attaching a ubiquitin residue at a nearby lysine or other residue (Haglund and Dikic, 2005; Gilkerson et al., 2015; McDowell and Philpott, 2013), it is plausible that the K899R lines exhibit normal responses because the phot1 protein is being ubiquitinated elsewhere. As we have shown there is no difference in detected ubiquitin with immunoprecipitated phot1GFP control versus our phot1K899R mutant lines, this hypothesis seems likely.

Unexpectedly we observed abnormal chloroplast accumulation in the K899R lines (Figure 2-10). As NPH3 is not involved in this phot1-mediated function, this was surprising. But as previously mentioned, RPT2 and NRL31/NCH1/SR1IP1, both members of the same protein family as NPH3, have been shown to be involved in chloroplast accumulation (Suetsugu et al., 2016). Furthermore, not only does RPT2 and NRL31/NCH1/SR1IP1 interact with phot1 (Inada et al., 2004; Suetsugu et al., 2016) but NRL31/NCH1/SR1IP1 has also been shown to interact with CUL3a (Zhang et al., 2014) which would make one of these two proteins potential candidates for phot1 ubiquitination in the chloroplast accumulation response. It is possible that lysine899, identified as the ubiquitination site in the phototropic response, may also be involved in phot1 ubiquitination in the chloroplast accumulation response but be targeted by a different CRL3 ${ }^{N R L}$ complex.

Additionally, lysine526, which was previously identified as a blue lightdependent phot1 ubiquitination site (Deng et al., 2014), may serve as a ubiquitination site in the chloroplast accumulation response as we observe the same bending response in seedlings harboring the phot1K526/527R substitution 
as those with the phot1K899R substitution, but observed varied phenotypes with respect to the chloroplast accumulation response. Moving forward, assessing phot 1 protein levels in leaves where we observe this loss of function is significant to begin to identify a role of ubiquitination of phot 1 in this response. 


\section{Chapter 3 Characterizing an allelic series of mutants within $\mathrm{NPH} 3$ to identify domain function}

\section{Introduction}

Plants are limited in their ability to gather resources as they are rooted into the ground. However, they have evolved several mechanisms to assist them to overcome this disadvantage. Phototropism is one mechanism that plants have evolved to maximize their photosynthetic light capture and nutrient in-take (Liscum et al., 2014). Phototropism, a plants ability to bend towards or away from a light source, is facilitated by the perception of blue light by the phototropin (phot) family of blue light photoreceptors (Briggs et al., 2001). In Arabidopsis thaliana, there are two phototropins, with phototropin1 functioning as the primary photoreceptor under low intensity light and phot and phot2 acting redundantly in high intensity light (Khurana and Poff, 1989; Liscum and Briggs, 1995; Sakai et al., 2001).

Equally critical for the phototropic bending response is a second protein, NPH3 (NONPHOTOTROPIC $\underline{H}$ YPOCOTYL으) (Liscum and Briggs, 1996) which has been shown to interact with phot 1 in light and dark conditions (Motchoulski and Liscum, 1999). In addition to phototropism, NPH3 is involved in photmediated leaf movement (Inada et al., 2004; Inoue et al., 2008b). NPH3 has been shown to be dephosphorylated by an unknown type 1 protein phosphatase (PP1), in a blue light- and phot-dependent manner, and this dephosphorylation is critical for the phototropic bending response (Pedmale and Liscum, 2007). 
Roberts and colleagues (2011) reported that NPH3 interacts with Cullin 3 (CUL3) proteins to act as an adapter protein in an E3 ubiquitin ligase complex, named CRL3 $^{\mathrm{NPH} 3}$ (ㅁLLIN $\underline{\mathrm{RING}}$ LIGASE3 $\underline{\mathrm{NPH}}$ ). This CRL3 ${ }^{\mathrm{NPH3}}$ complex is responsible for ubiquitinating phot1 (Roberts et al., 2011).

$\mathrm{NPH} 3$ interaction with phot1 and CUL3 occurs through two distinct proteinprotein interaction domains: an amino-terminal BTB (broad complex, tramtrack, and bric a brac) domain (Stogios et al., 2005) mediates interaction with CUL3, while, a carboxyl-terminal coiled coil (CC) domain mediates interaction with phot1 (Motchoulski and Liscum, 1999). Between these two domain lies a central 'NPH3 domain' (Pfam, PF03000) which is a conserved sequence domain contained within all 33 members of the NRL (NPH3-ㅁPT2-LIKE) protein family (Pedmale et al., 2010).

While only RPT2 (ㅁOOTPHOTOTROPISM르), has been shown to be involved in the phototropic pathway (Inada et al., 2004), NRL members have been shown to in involved in a variety of growth and development processes such as organ development, vein patterning, pollen germination, chloroplast movement among others (Petricka et al., 2008; Lalanne et al., 2004; Cheng et al., 2008; Suetsugu et al., 2016). Interestingly, two other NRL members, NRL3/

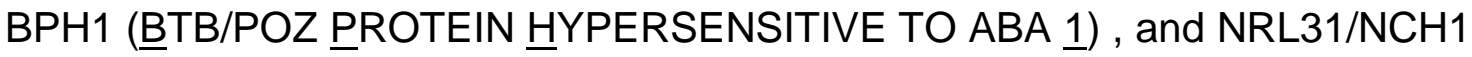
(NRL PROTEIN FOR $\underline{\mathrm{CH} L O R O P L A S T}$ MOVEMENT $\underline{1}$ )/ SR1IP1 (AtSR1 interaction protein 1) have been shown to interact with CUL3 and act as a substrate adapter in an E3 ubiquitin ligase complex (Suetsugu et al., 2016; Zhang et al., 2014; Woo et al., 2018) with NRL31/NCH1/SR1IP1 functioning in 
chloroplast movement, which is another phot-mediated function (Suetsugu et al., 2016).

CUL3 interacts with BTB domain-containing proteins (Genschik et al., 2013). It is interesting to speculate that the NRL family members (which all but two members) contain a BTB domain (Pedmale et al., 2010), may act as substrate adapters in an E3 ubiquitin ligase complex, and that the $\mathrm{CC}$ or 'NPH3 domain' may lend substrate specificity. Therefore, learning more regarding these domains may help identify function.

In many organisms it has been shown that missense mutations can result in temperature-sensitive phenotypes (Horowitz and Mitchell, 1950; Horowitz, 1950). A change in permissive conditions, for example, higher or lower temperature, might affect the structure and/or function of the protein containing a single amino acid change compared to that of the wildtype protein (Sandberg et al., 1995). This could occur due to a change in expression levels, or affect proper protein folding, or otherwise affect protein stability (Sandberg et al., 1995). We have utilized an allelic series of NPH3 missense mutants located throughout the NPH3 protein, generated by TILLING (Targeting Induced Local Lesions in Genomes) (McCallum et al., 2000; Till et al., 2003) to characterize the phototropic phenotype under permissive and non-permissive temperatures, in an attempt to relate that phenotype to particular domains within NPH3. 


\section{Materials and Methods}

\section{Plant Materials}

Arabidopsis thaliana seedlings used in this study were in the Columbia (col-0) ecotype but carry a homozygous erecta105 (er105) mutation. The erecta mutation does not influence phototropism and was utilized in design and execution of the TILLING mutagenesis scheme (Till et al., 2003). Several different alleles of NPH3 (nph3-10 through nph3-18) generated by TILLING were used, along with the null mutant nph3-6 (Motchoulski and Liscum, 1999). We also examined several different accessions of Arabidopsis that are part of the 1001 genomes collection (Alonso-Blanco et al., 2016). Lastly, used as a positive control for the transgenic plants generated, we used NPH3:mCHERRY line as described in Roberts et al, (2011). $n p h 3^{\text {TILING }}$ alleles and Arabidopsis thaliana ecotypes were obtained from the Arabidopsis Biological Stock Center. Ecotype seed stocks include: Var2-1 (CS), IP-Fun-0 (obtained kindly from Joy Bergelson), IP-Cad-0 (CS76739); IP-Mun-0 (CS77114); IP-Sne-0 (CS77258); IP-Vdt-0 (CS78838); IP-Ver-5 (CS78841); Benk-1 (CS22530); Ca-0 (C1060S), Kelsterbach-4 (CS6041), Li-7 (CS1330), Noveg-3 (CS77133), Bijisk-4 (CS9627), IST-29 (CS76948), IP-Moc-11 (CS77103), Jea (CS76148), IP-Vdm-0 (CS78837), Noveg-1 (CS77131); and nph3 ${ }^{\text {TILING }}$ allele seed stocks include: nph3-10 (CS87061), nph3-11 (CS87451), nph3-12 (CS87701), nph3-14 (CS90709), nph3-15 (CS86489), nph3-17 (CS80291), nph3-18 (CS91404). nph3 ${ }^{\text {TLLING }}$ allele seed stocks were subsequently genotyped for homozygosity by the use of CAPS or dCAPS 
markers (Konieczny and Ausubel, 1993) designed for each allele. Only homozygous lines were used in further experiments.

$n p h 3^{\text {TILLING }}$ alleles were transgenically recapitulated by using site directed mutagenesis (Thermo Scientific, Phusion Site-Directed Mutagenesis Kit). Primers were designed to introduce the single nucleotide polymorphism within the coding sequence of a $N P H 3 \mathrm{cDNA}$. Once mutated, using a gateway cloning strategy (Invitrogen) the sequence was fused to an mCHERRY (Shaner et al., 2004) and flag tag, flanked by the native NPH3 promoter and 3'UTR sequence. Using the floral dip method (Clough and Bent, 1998), these constructs were transformed into null-mutant nph3-6 plants. Screening plants for hygromycin resistance, as this gene is contained within the binary vector (pMDC111 backbone (Curtis and Grossniklaus, 2003)), homozygous lines were identified and used in further experiments.

\section{Growth and Light Conditions}

Seeds were sterilized with $30 \%$ bleach and spread on half strength Murashige and Skoog (MS) media plates with a thin layer of media on top. Plates were placed at 4 degrees Celsius in the dark for 2-4 days. After this vernalization period, seeds were subjected to a 1-hour red light treatment (yellow fluorescent bulbs, plates placed underneath and covered with a piece of red plexiglass) to stimulate uniform germination (Short and Briggs, 1990). Subsequently plates are placed in the dark at $22^{\circ} \mathrm{C}$ for 3 days prior to light treatment. 


\section{Hypocotyl Phototropism Measurements}

Three-day old etiolated seedlings are grown in darkness for 3 days at $22^{\circ}$ $\mathrm{C}$ and then exposed to 4 hours of unilateral blue light at a fluence rate of 0.1 $\mu \mathrm{mol} \mathrm{m} \mathrm{m}^{-2} \mathrm{~s}^{-1}$. After light treatment, seedlings are immobilized on transparency film, covered with transparent tape. Curvatures were determined from tracings of immobilized seedlings (Stowe-Evans et al., 2001). Data was plotted using average degrees of curvature along with standard error. A students T-test was used to determine statistical significance of means between genotypes in a given condition.

\section{Leaf Angle Measurements}

Plants were grown for 9 days under $50 \mu \mathrm{mol} \mathrm{m}^{-2} \mathrm{~s}^{-1}$ constant white light on promix potting soil mixed with Miracle-Gro water soluble all-purpose plant food (https://www.miraclegro.com) (1 tbsp per gallon of water) for additional nutrients. After 9 days, plants were transferred to red $\left(25 \mu \mathrm{mol} \mathrm{m} \mathrm{m}^{-2} \mathrm{~s}^{-1}\right)$ plus blue light $(0.2$ $\mu \mathrm{mol} \mathrm{m} \mathrm{m}^{-2} \mathrm{~s}^{-1}$ ) for 5 days (Inoue et al., 2008b). Subsequent pictures were taken of light treated seedlings and used in conjunction with Fiji Is Just ImageJ (FIJI) software to measure the petiole angle of the first true leaf. Angles were measured between the hypocotyl to the petiole, and subsequently subtracted from $90^{\circ}$ to determine the horizontal axis (see figure 2-6). Data was plotted by angle average (in degrees) along with the standard error. Again, a student's t-test was used to determine statistical significance. 


\section{Protein Extraction, SDS Page, and Immunoblot Analysis}

Three-day old seedlings grown as described above were either mock treated (darkness for 4 hours) or subjected to 4 hours of unilateral blue light at a fluence rate of $0.1 \mu \mathrm{mol} \mathrm{m}{ }^{-2} \mathrm{~s}^{-1}$. Following either mock or light treatment, seedlings were removed from plates and frozen in liquid nitrogen awaiting protein extraction. To extract total protein from seedlings, seedlings were ground in buffer described by Lariguet and colleagues (2006), spun down at 10,000 rpm, and the supernatant transfer to a new tube. Total protein was quantified by using Coomassie Protein Assay Kit (Thermo Pierce, catalog number 23200).

Total protein samples were separated on an $8 \%$ SDS-Page gel until the 72kDa standard contained in the Pageruler Prestained Ladder (ThermoFisher, catalog number 26616) was near the bottom of the gel. Separated proteins were then transferred to nitrocellulose by electrophoresis in a $20 \%$ methanol, trisglycine buffer at $250 \mathrm{~mA}$ overnight. Nitrocellulose blots are then incubated in $5 \%$ milk made in tris-buffered saline with $0.5 \%$ Tween 20 (TBST) for a period of at least 2 hours prior to immunodetection.

Antibodies raised again the C-terminal end of NPH3 (Motchoulski and Liscum, 1999) were used to detect NPH3 protein levels as well as phosphorylation status (1:6000 dilution ratio, in 1\% milk, TBST). Blots were incubated in primary antibody for a period of at least 2 hours, after which blots were rinsed 3 times in TBST for at least 5 minutes each. Following washing, blots were incubated in secondary antibody (goat anti-rabbit, Genscript, catalog number A00098, 1: 10,000 dilution ratio, in 1\% milk, TBST) for 1 hour. 
Afterwards, blots are detected with the use of Immobilon Western Chemiluminescent HRP Substrate (Millipore Sigma, catalog number WBKLS0500).

\section{Results}

$n p h 3^{\text {TLLING }}$ alleles Exhibit a Temperature-Sensitive Phototropic Phenotype.

A series of nph3 (nph3-10 through nph3-18) mutants were obtained from the Arabidopsis Biological Resource Center generated by TILLING (Targeting Induced Local Lesions in Genomes) (McCallum et al., 2000; Till et al., 2003). These mutants contain a single nucleotide polymorphism (SNP) within the genomic DNA sequence of $N P H 3$ resulting in a missense mutation (Figure 3-1). 


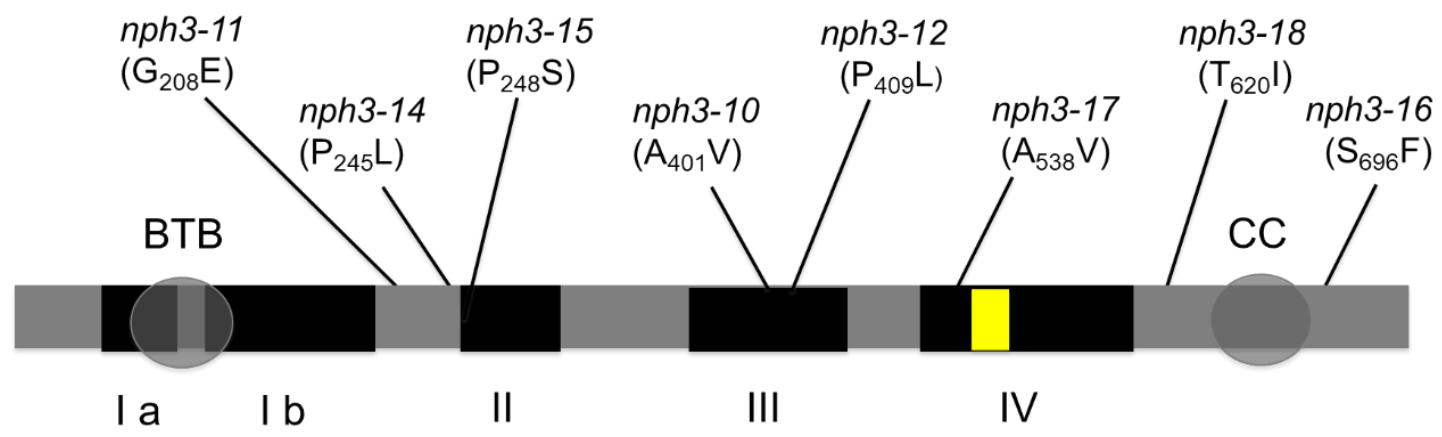

Figure 3-1 Position of nph3 $3^{\text {TLLING }}$ alleles on block diagram of NPH3

The black boxes represent conserved sequence domains within the NRL protein family, with the most conserved sequence identified in yellow (Motchoulski and Liscum, 1999). Gray ovals represent conserved structural domains and non-conserved regions are shown in light gray (Motchoulski and Liscum, 1999).

Position of missense TILLING (Till et al., 2003) alleles is denoted within this diagram and amino acid change is shown. 
Under permissive conditions, (room temperature), when subjected to low fluence $\left(0.1 \mu \mathrm{mol} \mathrm{m} \mathrm{m}^{-2} \mathrm{~s}^{-1}\right)$ unilateral blue light, with the exception of $n p h 3-16$, these alleles exhibit a partial loss of function, that is, they bend, but not equivalent to the positive control er105 (Figure 3-2). These results are not surprising given the nature of the mutations. However, when the same light treatment was applied to these mutants under non-permissive conditions $\left(10^{\circ} \mathrm{C}\right.$ rather than room temperature), we see the loss-of-function phototropic phenotype become more severe, again except for nph3-16 (Figure 3-3).

While these alleles are dispersed throughout most of the conserved and non-conserved domains of NPH3 (Figure 3-1), nph3-16 is of particular interest. It's location near the CC domain is interesting, but the nph3-16 mutation also affects a differentially phosphorylated serine within the protein, identified by MS/MS, which is phosphorylated in dark, dephosphorylated upon blue light exposure (Pedmale, 2008). Based on pharmacological studies, Pedmale and Liscum (2007) proposed that the dephosphorylated form of NPH3 represents the phototropically active form of the protein. If dephosphorylation of this particular serine ${ }^{696}$ is critical for the bending response, a mutation resulting in the inability to be phosphorylated would result in a constitutively dephosphorylated state leading to a phototropically 'sensitized' NPH3 protein. This may explain why we see a loss-of-function in the other alleles, but enhanced bending in nph3-16. 


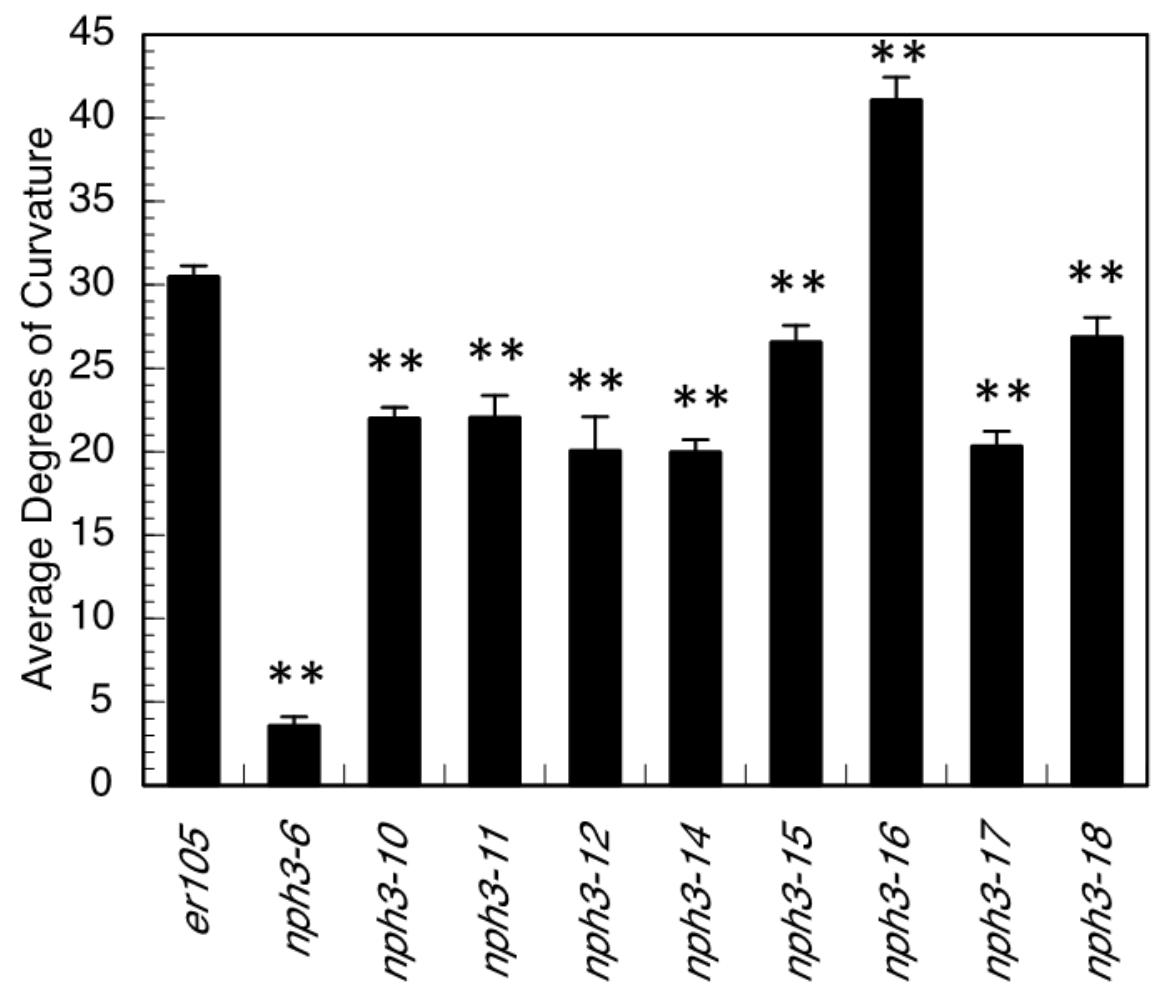

Figure 3-2 Hypocotyl phototropism of nph3 ${ }^{\text {TILLING }}$ alleles at room temperature

Hypocotyl phototropism of $n p h 3^{\text {TILLING }}$ alleles for 4 hours of unilateral blue light at a fluence rate of $0.1 \mu \mathrm{mol}$ $\mathrm{m}^{-2} \mathrm{~s}^{-1}$. Positive control represented by er105, and the null allele $n p h 3-6$ represents the negative control. Data is represented by average degrees of curvature with standard error denoted from three independent replicate experiments ( $n>73$ seedlings). Statistical significance determined by a students T-test, of mean average differing from that of er105 is denoted with two asterisks $(P<0.01)$. 


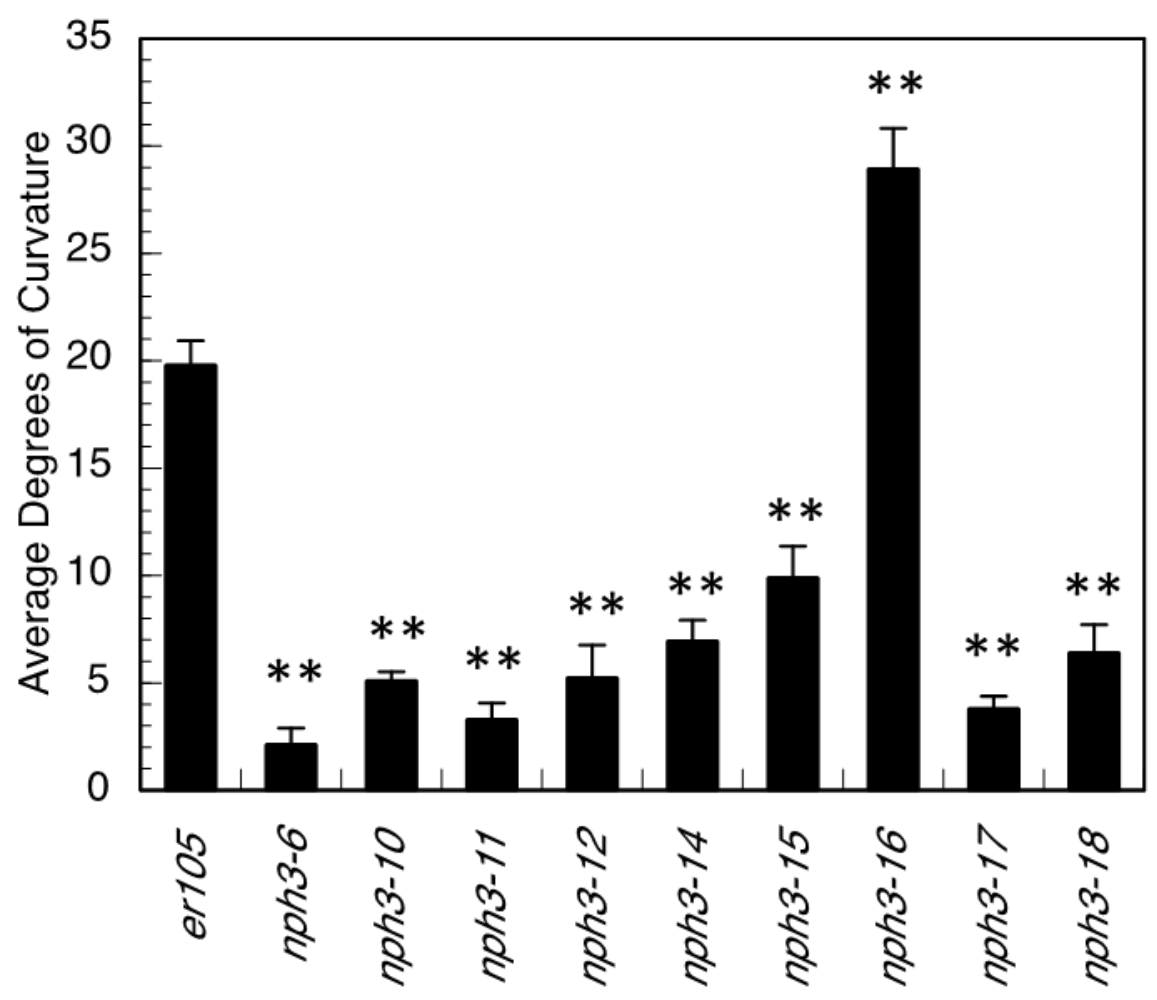

Figure 3-3 Hypocotyl phototropism under non-permissive temperature

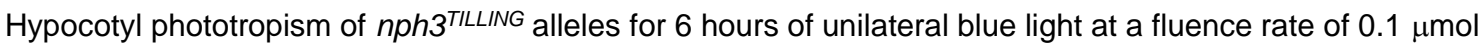
$\mathrm{m}^{-2} \mathrm{~s}^{-1}$ at $10^{\circ} \mathrm{C}$. Positive control represented by er105, and the null allele $n p h 3-6$ represents the negative control. Data is represented by average degrees of curvature with standard error denoted from three independent replicate experiments ( $\mathrm{n}>53$ seedlings). Statistical significance determined by a students T-test of mean average differing from that of er105 is denoted with two asterisks $(\mathrm{P}<0.01)$. 


\section{Phototropic Phenotypes of $n p 3^{\text {TILLING }}$ Alleles can be Recapitulated in Transgenic Seedlings.}

Due to the nature of EMS mutagenesis, the missense mutations within $\mathrm{NPH} 3$ (represented in Figure 3-1) designate one mutation, of likely several, within the Arabidopsis genome of these lines. In order to connect without doubt the phototropic phenotype of the $n p h 3^{\text {TILLING }}$ alleles to the mutations within $n p h 3^{\text {TILLING }}$ mutations, we attempted to transgenically recapitulate in an $n p h 3-6$ null-mutant background. Transgenic lines were selected that express similar $\mathrm{NPH} 3$ protein levels to that of the positive control NPH3:mCHERRY (Roberts et al., 2011) (Figure 3-4). In fact, transgenic TILLING allele lines appear to express higher levels of NPH3 than that of the NPH3:mCHERRY control (Figure 3-4).

These transgenic seedlings were exposed to 6 hours of low intensity $(0.1$ $\mu \mathrm{mol} \mathrm{m} \mathrm{m}^{-2} \mathrm{~s}^{-1}$ ) unilateral blue light at both room temperature (Figure 3-5) and under non-permissive conditions $\left(10^{\circ} \mathrm{C}\right)$ (Figure 3-6). At room temperature, similar to the original $n p h 3^{\text {TILLING }}$ alleles, the transgenic lines recapitulate the partial loss-of-function phototropic phenotype as compared to the positive control (Figure 3-5). However, under non-permissive conditions, $10^{\circ} \mathrm{C}$, we do not observe a recapitulation of the phenotype (Figure 3-6). While the observed phenotype is not significant, we do still observe the same trend in our recapitulated alleles as we do in the original $n p h 3^{\text {TILLING }}$ alleles. Additionally, we do not see a significant change in bending phenotype at non-permissive temperatures between our positive control and our null allele nph3-6, suggesting 
that our transgenic control is either affected by temperature more so than wildtype, or perhaps this may be due to the fact that our control transgenic seedlings at room temperature only recapitulate $50 \%$ of wild-type seedlings. While we do observe a decrease in average curvature in the wild-type seedlings as well, in our transgenic control seedlings, the average observed is already measuring closer to the null allele, therefore, as seedlings are affected by temperature, it seems likely that this may explain the lack of significance. A second explanation for this lack of significance between controls seedlings and transgenic lines at non-permissive temperatures could be due to the difference in protein expression (Figure 3-6). As observed, there is higher expression of NPH3 protein in the recapitulated TILLING seedlings than the control NPH3:mCHERRY line. At room temperature, or permissive temperature, this lower amount of protein expression might not affect these seedlings ability to bend towards the light; however, under non-permissive temperatures, this lower protein expression may affect seedlings ability to respond normally. 


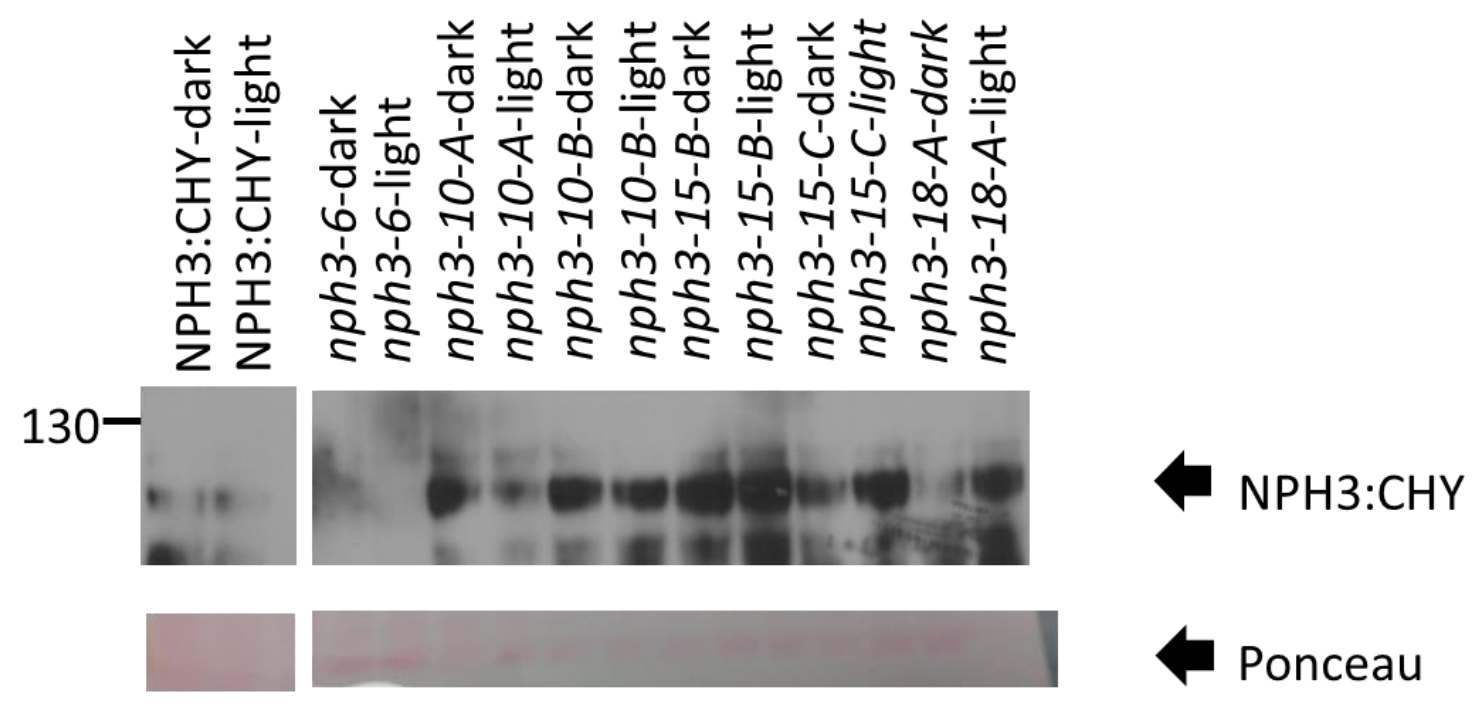

Figure 3-4 Recapitulated TILLING alleles express NPH3 protein

Seedlings were either mock treated or exposed to 4 hours of unilateral blue light at a fluence rate of 0.1 $\mu \mathrm{mol} \mathrm{m} \mathrm{m}^{-2} \mathrm{~s}^{-1}$. NPH3 total protein was detected by anti-NPH3 (Motchoulski and Liscum, 1999) dilution ratio 1:6000. Bottom panel represents a loading control. 


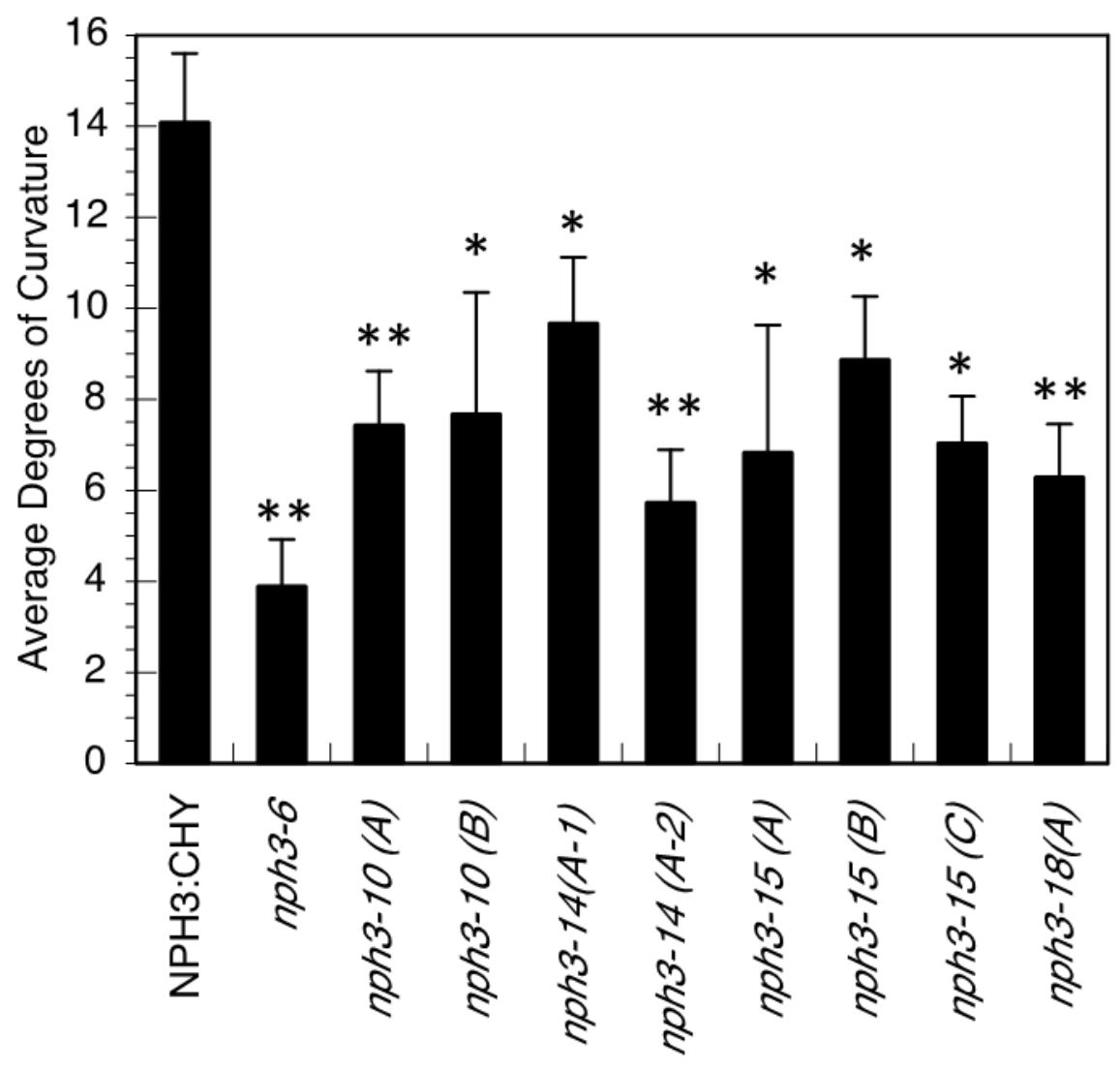

Figure 3-5 At room temperature transgenic lines recapitulate the phototropic phenotype of original TILLING alleles

Hypocotyl phototropism at room temperature of NPH3 transgenic lines for 6 hours of unilateral blue light at a fluence rate of $0.1 \mu \mathrm{mol} \mathrm{m} \mathrm{m}^{-2} \mathrm{~s}^{-1}$. Positive control represented by NPH3: CHY (Roberts et al, 2011) and the null allele nph3-6 represents the negative control. Data is represented by average degrees of curvature with standard error denoted from three independent replicate experiments ( $n>66$ seedlings). Statistical significance determined by a students T-test, of mean average differing from that of NPH3: $\mathrm{CHY}$ is denoted with an asterisk $(P<0.05)$, or two asterisks $(P<0.01)$. 


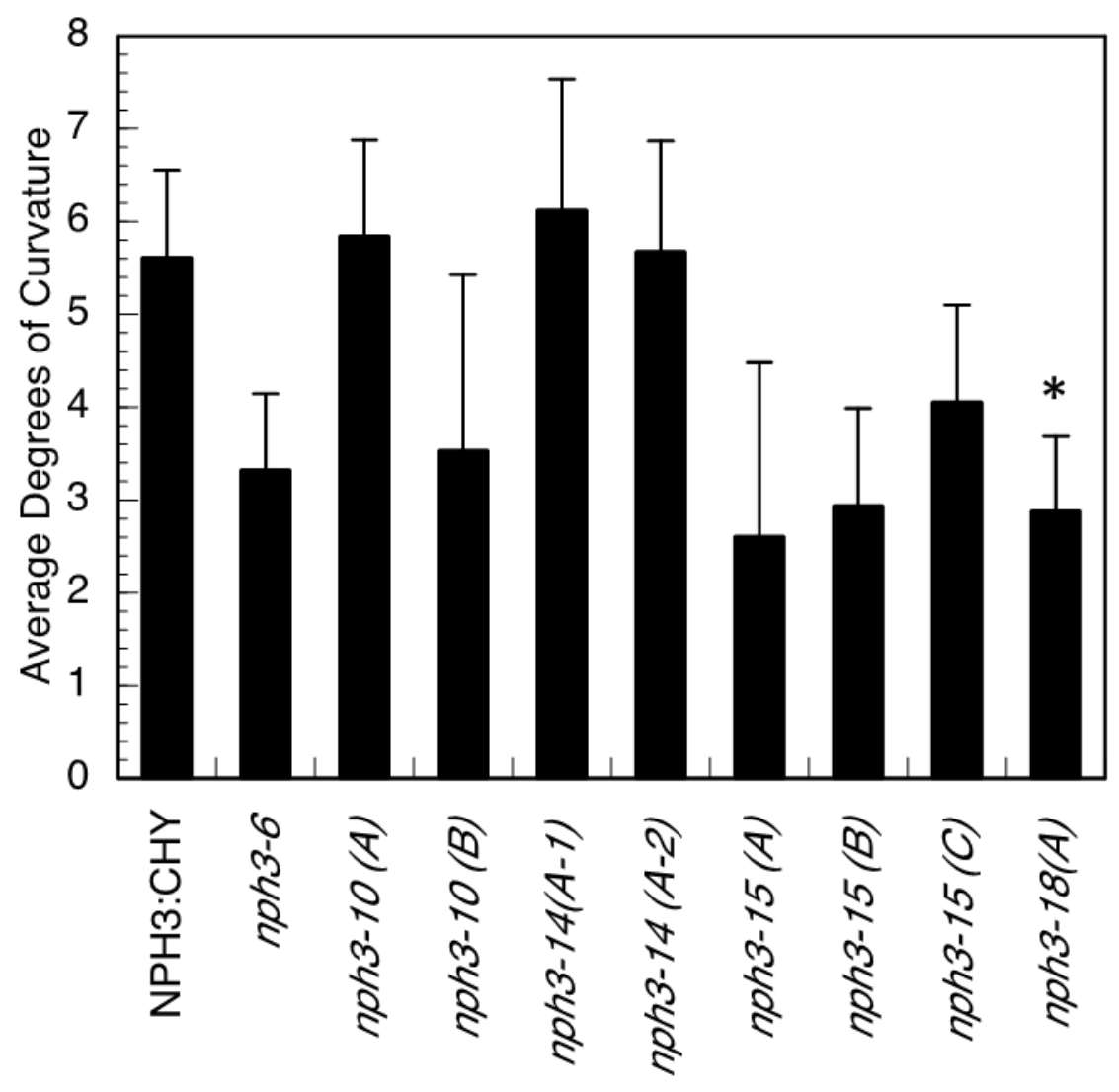

Figure 3-6 Hypocotyl phototropism under non-permissive conditions of recapitulated TILLING alleles Hypocotyl phototropism at $10^{\circ} \mathrm{C}$ of NPH3 transgenic lines for 6 hours of unilateral blue light at a fluence rate of $0.1 \mu \mathrm{mol} \mathrm{m} \mathrm{m}^{-2} \mathrm{~s}^{-1}$. Positive control represented by NPH3: $\mathrm{CHY}$ (Roberts et al, 2011) and the null allele $n p h 3-6$ represents the negative control. Data is represented by average degrees of curvature with standard error denoted from three independent replicate experiments ( $n>50$ seedlings). Statistical significance determined by a students T-test, of mean average differing from that of NPH3: $\mathrm{CHY}$ is denoted with an asterisk $(P<0.05)$. 


\section{Leaf Movement in Transgenic TILLING Lines}

In an effort to use these TILLING mutants to further characterize the conserved sequence domains within the NPH3 protein we investigated their phenotype in a second phot1-mediated function. As previously mentioned, NPH3 is involved in phot1-mediated leaf movement (Inada et al., 2004; Inoue et al., 2008a). Therefore, we examined petiole angles of transgenic seedlings carrying the TILLING mutations after red plus blue light treatment to observe if the leaf movement is defective in mutant plants (Figure 3-7). With the exception of nph318 , no significant change in petiole angle was observed in transgenic mutant seedlings, indicating that the single amino acid changes do not affect leaf movement function in plants at permissive temperatures. While we do observe a significant change in the phototropic phenotypes at permissive temperatures in transgenic seedlings, the phenotype is not a complete loss-of-function, indicating that it is possible we could see a significant change in leaf movement at nonpermissive temperatures. Moving forward, identifying whether this function is defective at non-permissive temperatures would further our characterization of this protein. 


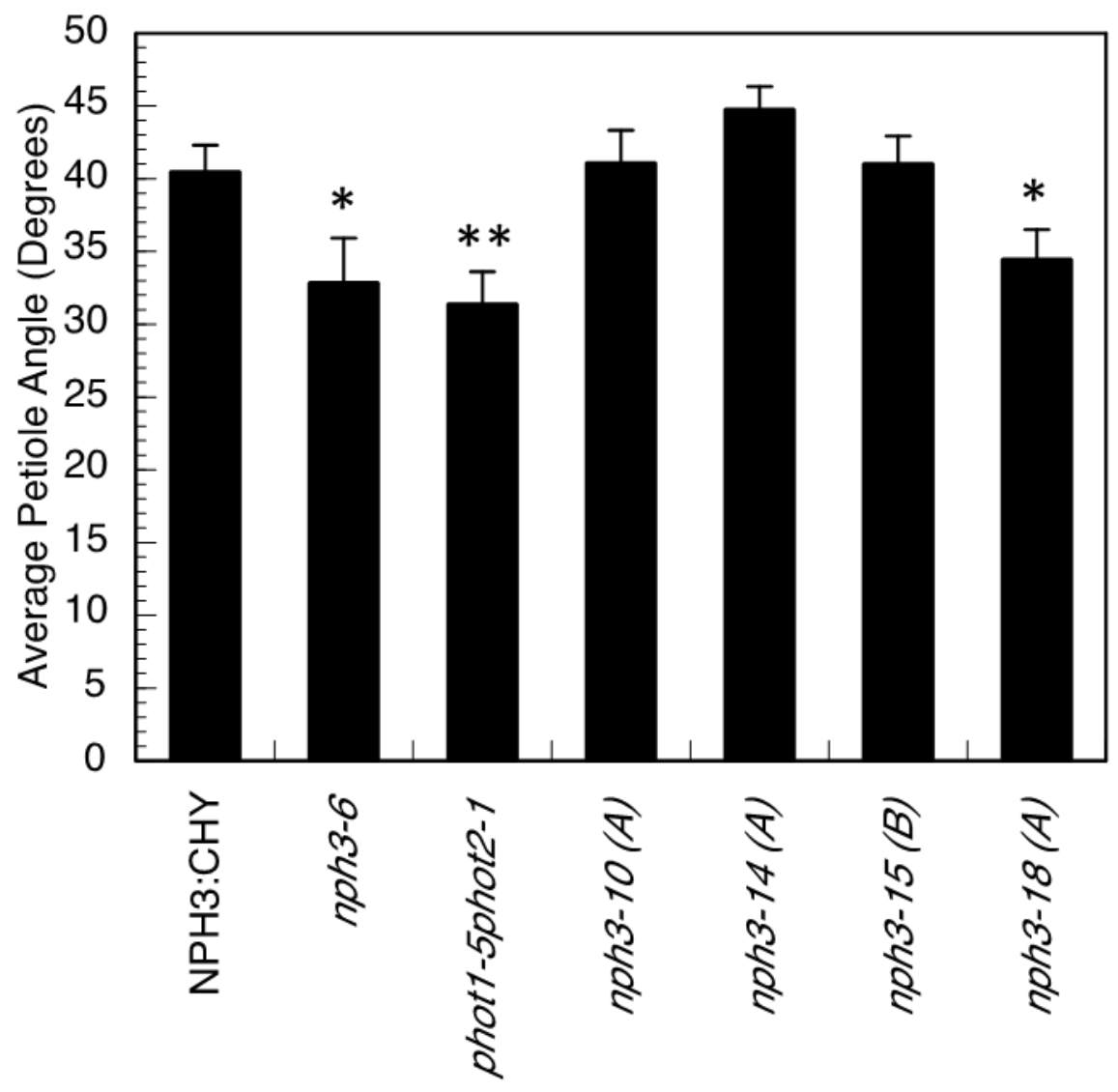

Figure 3-7 Petiole angle measurements of transgenic TILLING alleles

Leaf movement of NPH3 transgenic lines is identified by measuring petiole angle of seedlings in red (25 $\left.\mu \mathrm{mol} \mathrm{m} \mathrm{m}^{-2} \mathrm{~s}^{-1}\right)$ plus blue light $\left(0.2 \mu \mathrm{mol} \mathrm{m} \mathrm{m}^{-2} \mathrm{~s}^{-1}\right)$ for 5 days. Positive control represented by NPH3: CHY (Roberts et al, 2011) and the null allele nph3-6 as well as a phototropin double mutant (phot1-5phot2-1) represents the negative control. Data is represented by average petiole angle in degrees with standard error denoted from three independent replicate experiments ( $n>25$ seedlings). Statistical significance determined by a students T-test, of mean average differing from that of $\mathrm{NPH} 3: \mathrm{CHY}$ is denoted with an asterisk $(\mathrm{P}<$ 0.05), or two asterisks $(P<0.01)$. 


\section{Characterizing the Phototropic Phenotype of Arabidopsis thaliana Accessions}

With the use of 1001 genomes (Alonso-Blanco et al., 2016), Arabidopsis accessions containing single nucleotide polymorphisms (SNPs), relative to the Columbia (Col-0) accession, within the NPH3 sequence were identified. SNPs that occurred within few or only one accessions were chosen as those more likely to represent functional divergence within NPH3. SNP's within these accessions are dispersed throughout all conserved domains within NPH3 (Figure 3-8).

Arabidopsis accession seedlings exposed to 4 hours of low intensity $(0.1$ $\mu \mathrm{mol} \mathrm{m} \mathrm{m}^{-2} \mathrm{~s}^{-1}$ ) unilateral blue light results in a variety of phototropic phenotypes relative to the Col-0 accession (Figure 3-9). Three classes of phenotypes emerge from this screen. Several accessions exhibit a similar bending response to that of Col-0, one accession displays a significantly higher degree of curvature, and the majority exhibit a partial loss-of-function. Interestingly, those accessions with the same SNP do not present the same phenotype across the board, leading to the assumption that there may be a second site mutation within the genome responsible for the phenotype observed. 


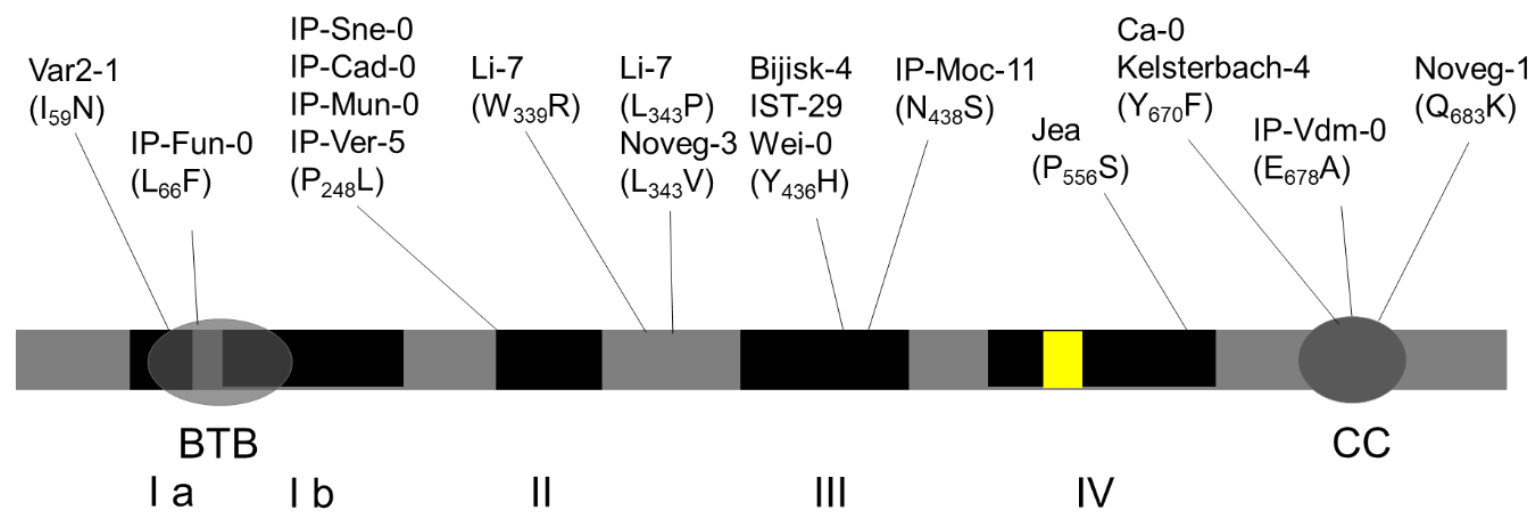

Figure 3-8 Location of NPH3 SNP within Arabidopsis thaliana ecotypes relative to Columbia Arabidopsis thaliana ecotypes which contain a SNP within the NPH3 sequence relative to Columbia. Location of SNP is denoted on the block diagram of NPH3 along with the amino acid change produced by SNP. 


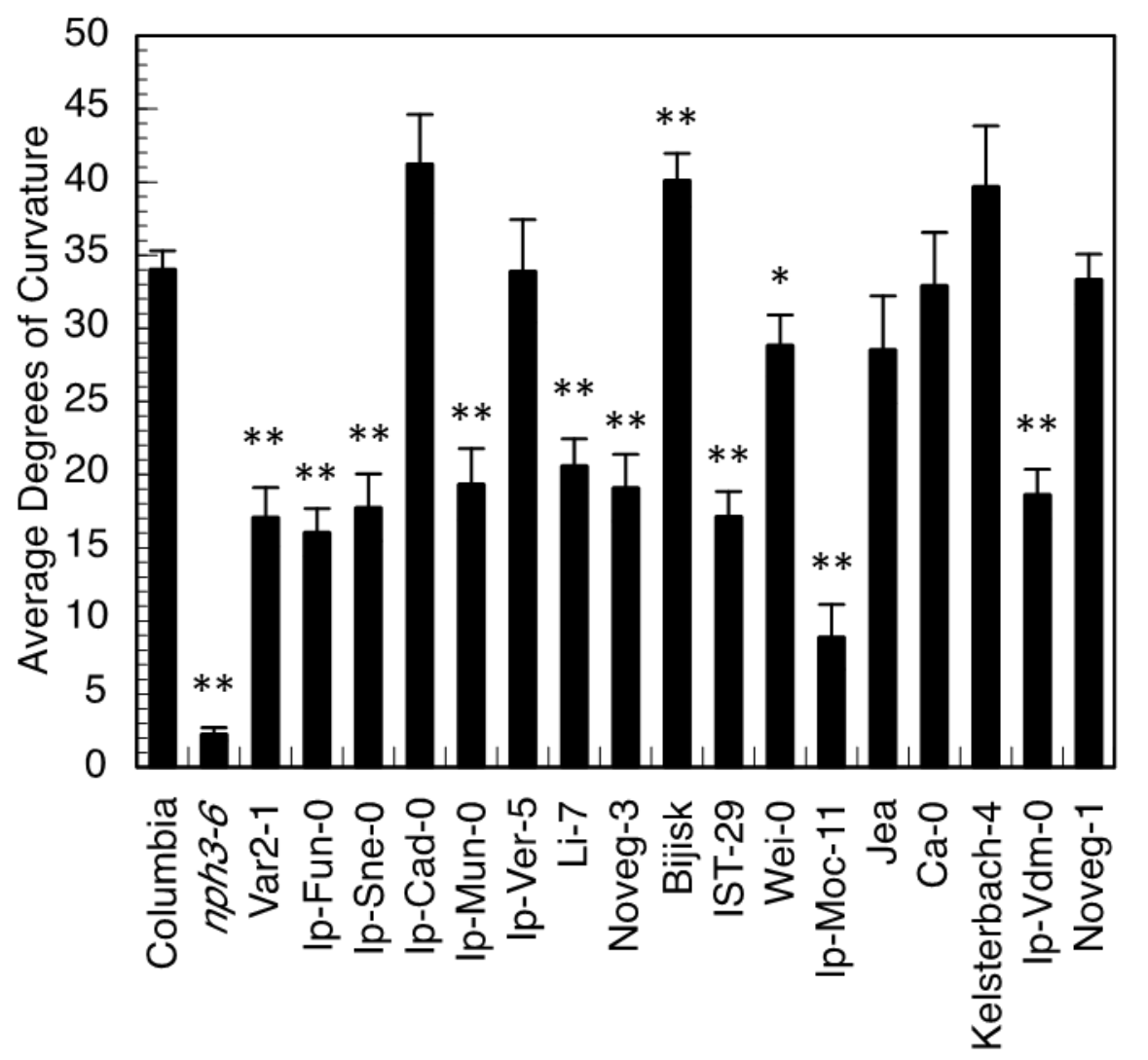

Figure 3-9 Hypocotyl phototropism on 3-day-old Arabidopsis thaliana ecotype seedlings

Hypocotyl phototropism at room temperature of Arabidopsis thaliana ecotypes for 4 hours of unilateral blue light at a fluence rate of $0.1 \mu \mathrm{mol} \mathrm{m} \mathrm{m}^{-2}$. The null allele $n p h 3-6$ represents the negative control. Data is represented by average degrees of curvature with standard error denoted from three independent replicate experiments. Statistical significance determined by a students T-test, of mean average differing from the Columbia accession line is denoted with an asterisk $(P<0.05)$, or two asterisks $(P<0.01)$. 


\section{Discussion}

Within the NPH3 protein, two protein-protein interaction domains occur which allow NPH3 to interact with phot1 and CUL3 (Motchoulski \& Liscum, 1999; Roberts, D., et al., 2011). A sequence specific domain referred to as the 'NPH3 domain' lies between these protein-protein interaction domains and is conserved within all 33 members of the NRL protein family (Pedmale et al., 2010). Function of this sequence specific domain is unknown at this time. In an effort to further characterize domains within the NPH3 protein, we described the phototropic phenotype in a series of induced mutants and naturally occurring Arabidopsis accessions which containing a SNP within NPH3 relative to the Columbia accession.

Temperature-sensitive mutants have been well documented in many organisms (Horowitz and Mitchell, 1950; Horowitz, 1950; Ben-Aroya et al., 2010), thus allowing one to assess protein function by using missense mutants. With the exception of $n p h 3-16$, the TILLING alleles represent a collection of temperaturesensitive mutants within NPH3. With regard to the original TILLING alleles except nph3-16, it's interesting to look at the individual amino acid changes which lead to a phenotypic change within these seedlings. Changes include polar side chain to a nonpolar side chain, and vice versa, polar to acid side chain, and a change resulting in loss of an aromatic ring. However, it's interesting to denote that no matter the change, the same loss-of-function phenotype in either permissive or non-permissive temperatures is observed. However, while the average curvature response is significantly different under permissive conditions, we still observe 
bending in seedlings carrying these mutations. But, at non-permissive temperatures, the average degrees of curvature for these mutant seedlings is reduced far more dramatically, leading to the conclusion that under nonpermissive temperatures, these mutations have a greater effect on protein expression, stability or folding. Additionally, with the exception of nph3-16, these amino acid substitutions occur throughout the NPH3 domain leading us to the conclusion that this domain may in fact lead to function specificity.

While we were able to recapitulate the phenotype transgenically at permissive temperatures, under non-permissive temperatures, we did not see a significant difference in phenotype as compared to our control NPH3:mCHERRY. The level of NPH3 protein expression observed within our transgenic mutants is higher than in our positive control. This may imply that the lower level of expression at permissive temperatures is enough for normal response, but at non-permissive temperatures normal function cannot occur with this lower protein expression. The higher level of NPH3 protein expressed within our transgenic TILLING lines may close the gap between the observed phototropic phenotype compared to our positive control. It still needs to be determined whether these mutations lead to a change in the ability of NPH3 to interact with either phot 1 and/or CUL3, and whether the function of the CRL $3^{\mathrm{NPH} 3}$ ubiquitin ligase complex is altered.

An exciting and unexpected discovery in this study is the enhanced phototropic phenotype of $n p h 3-16$ compared to the other $n p h 3^{\text {TILLING }}$ alleles that exhibit a loss-of-function. As previously mentioned, NPH3 is a phospho-protein 
and dephosphorylation has been shown to be critical for the bending response (Pedmale and Liscum, 2007). Furthermore, Pedmale (2008) identified by MS/MS several differential phosphorylation sites dependent on light condition and serine $^{696}$ represents one such site which was shown to be phosphorylated only in dark conditions. This serine is substituted for a phenylalanine in the nph3-16 allele. This substitution renders this site unable to be phosphorylated, resulting in a constitutive 'dephosphorylated' state at site 696 . The bending response of this allele supports the hypothesis that the dephosphorylated state of the NPH3 protein is the functional state. If indeed serine ${ }^{696}$ represents the critical phosphorylation site within the protein we would expect this response in permissive and non-permissive conditions. In order to further assess if serine ${ }^{696}$ is the critical phosphorylation site, we have generated transgenic seedlings carrying a substitution for this serine to an aspartic acid, to mimic a constitutively 'phosphorylated' state to further characterize phosphorylation status. Again, if this serine ${ }^{696}$ is critical for the phototropic bending response, we should observe a loss-of-function in seedlings carrying this substitution, the opposite phenotype to that of $n p h 3-16$.

Lastly, naturally occurring Arabidopsis thaliana accessions were obtained that carry a SNP within NPH3 relative to the Columbia accession. Interestingly, three classes of bending responses were observed: accessions that respond similarly to Col-0, one accession that has an increased bending response, and

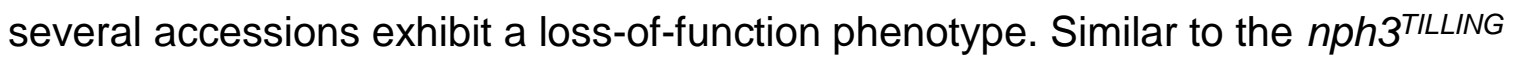
alleles, we see a variety of amino acid changes in these substitutions which may 
explain the phenotypes observed, and these SNP's occur throughout the domains of NPH3. Two ecotypes which exhibit a loss-of-function response, Var21 and IP-Fun-0, both occur within the BTB domain, which facilitates interaction with CUL3. These amino acid substitutions include a nonpolar side change for a polar side chain, or a nonpolar side chain for a nonpolar side chain which has a large aromatic ring. We speculate that these changes and their location may result in reduced ability of the protein to interact with CUL3, which is necessary for the phototropic bending response (Roberts et al., 2011). A similar conclusion can be made with regard to IP-Vdm-0, which contains a substitution in the CC domain, the interaction domain with phot1. This amino acid change substitutes a charged amino acid for nonpolar alanine, which may inhibit NPH3s ability to interact with phot 1 resulting in a loss-of-function response. Interestingly, the same SNP occurs in a few accessions, and we do not see the same phenotype in those seedlings, leading to the assumption that there may be a second site mutation within another gene responsible for the phenotype observed. While it's interesting to make these conclusions with regards to phototropic phenotype and $\mathrm{NPH} 3$ amino acid change, it still remains that there are other amino acid changes within the genome of these ecotypes. In order to fully assess phenotypically these substitutions, within $\mathrm{NPH} 3$, they need to be transgenically recapitulated within the null nph3-6 allele. Additionally, protein-protein interaction between $\mathrm{NPH} 3$ and CUL3 or phot1 can then be assessed as well as the functionality of the CRL3 ${ }^{\mathrm{NPH} 3}$ complex. 


\section{Chapter 4 Conclusions and Future Directions}

\section{Introduction}

Plants are rooted in the ground, and therefore are limited in their ability to gather necessary resources, such as water and light energy for photosynthesis. One mechanism plants have developed to aid in this limitation is phototropism, a plants ability to bend towards or away from a light source (Liscum et al., 2014). Phototropism is initiated through light perception by the phototropin (phot) family of blue light photoreceptors (Briggs et al., 2001). Arabidopsis thaliana contains two phots: phot 1 is the primary photoreceptor under low intensity blue light, while phot2 acts redundantly with phot1 under high intensity blue light (Briggs et al., 2001). In addition to phototropism, the phots mediate several other functions associated with optimization of photosynthesis, such as chloroplast movement, stomatal opening, and leaf movement and expansion (Christie, 2007).

Phototropin is characterized by an amino-terminal light sensory domain, and carboxyl-terminal protein kinase output domain (Huala et al., 1997; Christie et al., 1998) The sensory domain has associated with it two flavin mononucleotide (FMN) co-factors that act as light-absorbing (Christie et al., 1998). While the FMN associate with the sensory domain non-covalently in darkness, these co-factors form covalent adducts with conserved cystine residues in response to blue light absorption, which in turn leads to a conformational change in phot1 resulting in autophosphorylation/transphosphorylation (Christie et al., 1998; Salomon et al., 
2000; Sullivan et al., 2008). This autophosphorylation has been shown to be necessary for the phototropic bending response (Inoue et al., 2008a; Sullivan et al., 2008; Kaiserli et al., 2009).

A second protein shown to be critical for the phototropic bending response is NPH3 (NONPHOTOTROPIC $\underline{\text { HYPOCOTYL }}$ ) (Liscum and Briggs, 1996; Motchoulski and Liscum, 1999). In addition to phototropism, NPH3 is involved in phot1-mediated leaf movement (Inoue et al., 2008b). The NPH3 protein is characterized by an amino-terminal BTB (ㅁoad complex, tramtrack, and bric a brac) domain (Stogios et al., 2005), a central 'NPH3 domain' (Pfam, PF03000) and a carboxyl-terminal coiled-coil (CC) domain (Motchoulski and Liscum, 1999; Pedmale et al., 2010). Both phot1 and NPH3 have been shown to be plasma membrane-localized (Sakamoto and Briggs, 2002; Motchoulski and Liscum, 1999) and further, through the CC domain, NPH3 has been shown to interact with phot1 (Motchoulski and Liscum, 1999; Lariguet et al., 2006; Inada et al., 2004).

$\mathrm{NPH} 3$ is a phosphoprotein that is rapidly dephosphorylated upon blue light exposure by an unidentified type 1 protein phosphatase (Pedmale and Liscum, 2007). Not only is this dephosphorylation blue light-dependent, but it is also phot1-dependent (Pedmale and Liscum, 2007). Pedmale and Liscum (2007) proposed that it is this dephosphorylated state of NPH3 which is the active signaling form in the phototropic pathway.

Roberts and colleagues (2011), identified that CUL3 (CULLIN3) is involved in the phototropic bending response and interacts with NPH3 through its 
BTB domain. This interaction facilitates formation of an E3 ubiquitin ligase complex, designated CRL3 ${ }^{\mathrm{NPH} 3}$ (CULLIN RING LIGASE3 ${ }^{\mathrm{NPH} 3}$ ), which ubiquitinates phot1 in response to blue light (Roberts et al., 2011). However, ubiquitination type differed under low and high light conditions: under high light, phot1 is monoubiquitinated and poly-ubiquitinated, resulting in protein degradation by the $26 \mathrm{~S}$ proteasome. In contrast, under low light, phot 1 is solely monoubiquitinated (Roberts et al., 2011). A resent study has shown that this monoubiquitination promotes retention of dispersed phot1 at the plasma membrane as a means to facilitate signaling (Askinosie, 2016). Roberts (2013) mapped ubiquitination sites within phot1 and identified one site which was shown to be blue light- and NPH3-dependent: K899. A second ubiquitination site independently reported by Deng and colleagues (2014), K526, was also identified but shown to be blue light-dependent, but NPH3-independent.

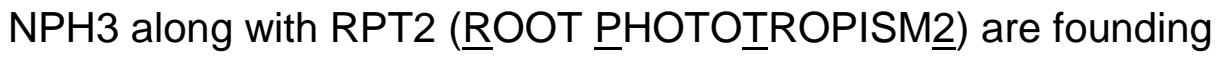
members of a 33-protein member family called NRL (NPH3-ㅁPT2 ㄴIKE) (Pedmale et al., 2010). Their general structure is similar, as they all contain the central 'NPH3 domain', two thirds contain the CC domain like NPH3, and all but two members contain the BTB domain (Pedmale et al., 2010). RPT2 has been shown to function in phototropism, (Okada and Shimura, 1992; Sakai et al., 2000). Other NRL family members have been shown to be involved in a variety of growth and development functions (Christie et al., 2017). Most interesting is the involvement of RPT2 and NRL31/NCH1 (NRL PROTEIN FOR CHLOROPLAST MOVEMENT 1)/ SR1IP1 (AtSR1 interaction protein 1) in phot1- 
mediated chloroplast accumulation (Suetsugu et al., 2016), as well as the fact that both these proteins interact with phot1 (Inada et al., 2004; Suetsugu et al., 2016). Furthermore, NRL31/NCH1/SR1IP1 as well as recently identified NRL3/

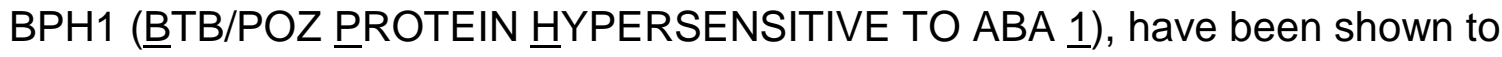
interact with CUL3 to form E3 ubiquitin ligase complexes (Woo et al., 2018; Zhang et al., 2014). It's interesting to speculate that BTB domain-containing NRL members may function as substrate adapters in CRL3s for a variety of different responses.

\section{Conclusions and Future Directions}

\section{Further Characterize Blue Llight-Dependent phot1 Ubiquitination on phot1- Mediated Phototropism and Chloroplast Accumulation}

A characteristic of ubiquitination is that it's been shown to be promiscuous.

That is, ubiquitin molecules can be attached to a nearby lysine if the native ubiquitination site is unavailable (Haglund and Dikic, 2005). This represents a possible explanation for the phototropic and immunoblot results with our transgenic phot1K899R lines. Phot1 contains 80 lysines within the amino acid sequence (Figure 4-1) so there are a number of alternative ubiquitination sites if K899 is unavailable. Additionally, when all lysines are mutated to arginine, we still observe ubiquitination in phot $1 K^{*} R$ lines (Figure 2-14, 2-15). As it has been reported that ubiquitination can occur at serine, threonine as well as cysteine in addition to lysine (Stringer and Piper, 2011; McDowell and Philpott, 2013; Gilkerson et al., 2015). An alternative approach to characterize the role of phot1 ubiquitination that addresses promiscuous ubiquitination at a nearby lysine or 
other residue, is utilizing deubiquitinating enzymes (DUbs) (Stringer and Piper, 2011) Stringer and Piper (2011) have reported that the catalytic domain of a DUb can be fused to a protein thus rendering it unable to be ubiquitinated. In light of our results, it seems as if this alternative approach will allow us to characterize physiological function of phot 1 in a ubiquitin-deficient mutant.

One outcome of this study which was unexpected, was the loss-offunction chloroplast accumulation phenotype we observed in the phot 1 K899R lines as well as some of the phot1K526-527R lines. As NPH3 has been shown to have no role in this response, mutating a NPH3-dependent ubiquitination site and observing a loss-of-function was surprising. In the future, identifying and comparing phot1 protein expression as well as phot1 ubiquitination via immunoprecipitation and immunoblot analysis in rosette leaves of phot1GFP control as well as phot $1 K 899 R$ and phot $1 K 526 / 527 R$ is critical to make sense of this observed phenotype.

As previously mentioned, RPT2 and NRL31/NCH1/SR1IP1 both interact with phot1 (Suetsugu et al., 2016; Inada et al., 2004) and have been shown to function in phot1-mediated chloroplast accumulation (Suetsugu et al., 2016). Additionally, Zhang and colleagues (2014) found that NRL31/NCH1/SR1IP1 can interact with CUL3a. It is possible that one of these proteins functions as a substrate adapter in a CRL3 complex involved in phot1 ubiquitination in blue light-dependent chloroplast accumulation, and that either K899 or K526 represents the ubiquitination site in this function. To further evaluate if RPT2 or NRL31/NCH1/SR1IP1 is involved in blue light-dependent phot1 ubiquitination in 
chloroplast accumulation, loss-of-function mutants of RPT2 or NRL31/NCH1/SR1IP1 can be crossed with phot1GFP (Sakamoto and Briggs, 2002) to assess phot1 ubiquitination in the absence of RPT2 or NRL31/NCH1/SR1IP1. 
1. MEPTEKPSTK PSSRTLPRDT RGSLEVFNPS TQLTRPDNPV FRPEPPAWQN

51 LSDPRGTSPQ PRPQQEPAPS NPVRSDQEIA VTTSWMALKD PSPETISKKT

101 ITAEKPQKSA VAAEQRAAEW GLVLKTDTKT GKPQGVGVRN SGGTENDPNG

151 KKTTSQRNSQ NSCRSSGEMS DGDVPGGRSG IPRVSEDLKD ALSTFQQTFV

201 VSDATKPDYP IMYASAGFFN MTGYTSKEVV GRNCRFLQGS GTDADELAKI

251 RETLAAGNNY CGRILNYKKD GTSFWNLLTI APIKDESGKV LKFIGMQVEV

301 SKHTEGAKEK ALRPNGLPES LIRYDARQKD MATNSVTELV EAVKRPRALS

351 ESTNLHPFMT KSESDELPKK PARRMSENVV PSGRRNSGGG RRNSMQRINE

401 IPEKKSRKSS LSFMGIKKKS ESLDESIDDG FIEYGEEDDE ISDRDERPES

451 VDDKVRQKEM RKGIDLATTL ERIEKNFVIT DPRLPDNPII FASDSFLELT

501 EYSREEILGR NCRFLQGPET DLTTVKKIRN AIDNQTEVTV QLINYTKSGK

551 KFWNIFHLQP MRDQKGEVQY FIGVQLDGSK HVEPVRNVIE ETAVKEGEDL

601 VKKTAVNIDE AVRELPDANM TPEDLWANHS KVVHCKPHRK DSPPWIAIQK

651 VLESGEPIGL KHFKPVKPLG SGDTGSVHLV ELVGTDQLFA MKAMDKAVML

701 NRNKVHRARA EREILDLLDH PFLPALYASF QTKTHICLIT DYYPGGELFM

751 LLDRQPRKVL KEDAVRFYAA QVVVALEYLH CQGIIYRDLK PENVLIQGNG

801 DISLSDFDLS CLTSCKPQLL IPSIDEKKKK KQQKSQQTPI FMAEPMRASN

851 SFVGTEEYIA PEIISGAGHT SAVDWWALGI LMYEMLYGYT PFRGKTRQKT

901 FTNVLQKDLK FPASIPASLQ VKQLIFRLLQ RDPKKRLGCF EGANEVKQHS

951 FFKGINWALI RCTNPPELET PIFSGEAENG EKVVDPELED LQTNVF

Figure 4-1 phot1 protein sequence

Amino acid sequence of phot1. Yellow highlighted boxes represent lysines, possible ubiquitination sites, within the sequence. Figure adapted from Roberts, 2013. 


\section{Identify Phosphorylation Site(s) Within NPH3 Critical to Phototropism}

Pedmale and Liscum (2007) previously identified the active, signaling state of NPH3 in the phototropic pathway is the dephosphorylated form. Using MS/MS phosphoproteomics approach, Pedmale (2008) identified 10 sites within $\mathrm{NPH} 3$ that are phosphorylated in the dark, but dephosphorylated upon blue light exposure (Figure 4-2). The tilling allele nph3-16 represents one of these previously identified sites (Figure 4-2). As our results suggest, the phototropic phenotype of this allele differ from that of the other TILLING alleles. The amino acid substitution in this allele is a phenylalanine for the native serine $\left(\mathrm{S}^{696} \mathrm{~F}\right)$. This substitution changes the structure of that particular residue, resulting in its inability to be phosphorylated, leading to a site mimicking a constitutively dephosphorylated state. This result lends further evidence to support Pedmale and Liscum's (2007) hypothesis that the active form of NPH3 is the dephosphorylated form as this allele is hyper-phototropic rather than hypophototropic.

The next step is to confirm that this serine ${ }^{696}$ represents the critical phosphorylation site within NPH3 in phototropism. We have generated transgenic plants containing a $\mathrm{S}^{696} \mathrm{D}$ substitution within $\mathrm{NPH} 3$ fused to $\mathrm{mCHERRY}$ as well as the native NPH3 promoter and 3'UTR. This substitution will accomplish the opposite of the nph3-16 substitution, if it represents the critical phosphorylation site, a loss-of-function phototropic phenotype should be observed. Homozygous lines have been identified and are awaiting analysis. Secondly, transgenically recapitulating the $n p h 3-16$ allele will confirm the gain-of-function phenotype seen 
in nph3-16 plants. Observing phosphorylation state in immunoblot analysis along with phototropic phenotype of both these lines should support $S^{696}$ as the critical phosphorylation site in the phototropic pathway. 
1 MMWESESDGG VGVGGGGGRE YGDGVLSSNK HGGVKTDGFE LRGQSWFVAT

51 DIPSDLLVKI GDMNFHLHKY PLLSRSGKMN RLIYESRDPD PTILILDDLP

101 GGPEAFELA KFCYGVPVDL TATNISGLRC AAEYLEMTED LEEGNLIFKT

151 EAFLSYVVLS SWRDSILVLK SCEKLSPWAE NLQIVRRC E IIAWKACSNP

201 KGIRWAYTGK APSP TNFA GSSPRWNESK DSSFYCSPSR NTNSQPVPPD

251 WWFEDVSILR IDHFVRVITA IKVKGMRFEL LGAVIMHYAG KWLPGLIKEG

301 GVAIAPAMSS AIGGGLGLGG DEMSISCGSN SSGGSSGPDW KGGLHMVLSA

351 GKTNGHQDSV ACLAGLGISP KDQRMIVESL ISIIPPQKDS VTCSFLLRLL

401 RAANMLKVAP ALITELEKRV GMQFEQATLQ DLLIPGYNNK GETMYDVDLV

451 QRLLEHFLVQ EQTEGSSPSR MSPSPSQSMY ADIPRGNNNN GGGGGGNNQN

501 AKMRVARLVD SYLTEVARDR NLPLTKFQVL AEALPESART CDDGLYRAID

551 SYLKAHPTLS EHERKRLCRV MDCQKLSMDA CMHAAQNERL PLRVVVQVLF

601 SEQVKISNAL ANTSLKEST】 LGEAMGTYQP MIPNRKTLIE ATPQSFQEG $\underline{\underline{W}}$

651 AAAKKDINTL KFELETVKTK YVELQNEMEV MQRQFEKTGK VKNTSSAW

701 TSGWKKLSKL TKMSGQE HD I GGEQAGV DHPPPRKPRR WRNSIS

Figure 4-2 NPH3 protein sequence

NPH3 protein sequence: BTB domain indicated by single underline, $\mathrm{CC}$ domain indicated by double underline and NPH3 domain (I-IV) indicated in bold (Motchoulski and Liscum, 1999). Sites phosphorylated in darkness highlighted in blue and sites phosphorylated in light highlighted in yellow (Pedmale, 2008). S696 indicated by circle. Figure from Roberts et al., 2013. 


\section{Further Characterize NPH3 and Its Role in the CRL3 ${ }^{\mathrm{NPH} 3}$ Complex and phot1 Ubiquitination}

An allelic series of NPH3 mutants: $n p h 3^{\text {TLLING }}$ alleles, with the exception of $n p h 3-16$, represent temperature sensitive mutants. It has previously been reported that a single amino acid change can affect protein stability, folding and/or expression at non-permissive temperatures (Sandberg et al., 1995). Original alleles as well as recapitulated transgenic lines display a significant difference in their phototropic bending response upon blue light exposure at permissive temperatures (room temperature). Under non-permissive temperatures we see this same trend, that is, our temperature sensitive alleles exhibit a decrease in their bending response, exhibiting a phenotype similar to our null allele nph3-6. NPH3 protein levels for all recapitulated TILLING alleles show higher levels of expression than that of our positive control NPH3:mCHERRY, which provides a possible explanation for why our phototropic non-permissive temperature results are not significantly different. Possibly this lower level of protein expressed in the positive control represents a level of expression which at permissive temperatures is able to function normally; however, under non-permissive temperatures, this lower protein expression is not enough to function normally, paired with the higher amount of protein expression in our transgenic lines we do not see a significant change in bending response. This leads to the conclusion that these amino acid substitutions may indeed be affecting protein stability, structure and/or function at non-permissive temperatures. 
To address whether these $n p h 3^{\text {TILLING }}$ alleles affect the function of NPH3 as a substrate adapter in a CRL3 ${ }^{\mathrm{NPH} 3}$ ubiquitin ligase complex, we need to first assess protein-protein interaction of the mutant $\mathrm{NPH} 3$ protein with phot1 and CUL3. We will immunoprecipitate NPH3 by using ANTI-FLAG ${ }^{\circledR}$ M2 Affinity Gel (Sigma A2220) and immunoblot with native phot1 antibody (Christie et al., 1998) to assess NPH3-phot1 interaction. Next it will be critical to assess the function of the CRL3 ${ }^{\mathrm{NPH} 3}$ complex. Assuming there is interaction between NPH3, phot1 and CUL3, we can immunoprecipitate the NPH3-phot1-CUL3 complex and assess phot1 ubiquitin levels by immunoblot analysis using a ubiquitin specific antibody. Interestingly, the Arabidopsis accessions lines identified with a unique single nucleotide polymorphism (SNP) within NPH3 compared to the accession line Col-0 display varying phototropic phenotypes. That is, at permissive temperatures, either their bending response is similar to Col-0, or we observe a hyper- or hypo-phototropic response. Of particular interest is IP-Fun-0 and Var21 , as both of these contain amino acid substitutions within the BTB domain. As this domain within NPH3 is where interaction with CUL3 occurs (Roberts et al., 2011), we may speculate that these substitutions may interfere with NPH3-CUL3 interaction, which may influence the function of the CRL3 ${ }^{\mathrm{NPH} 3}$ complex. Additionally, it's interesting to observe differing bending responses within accession lines containing the same SNP. This may imply a second-site mutation may occur in a gene yet to be identified in this mechanism. Interestingly, preliminary results suggest that the temperature sensitive nature of the TILLING alleles is not observed within Arabidopsis accession lines (Figure 4-3). More 
experiments need to be performed to confirm this result on displayed accession lines as well as other lines in which phototropic phenotype at non-permissive temperature has yet to be determined. 


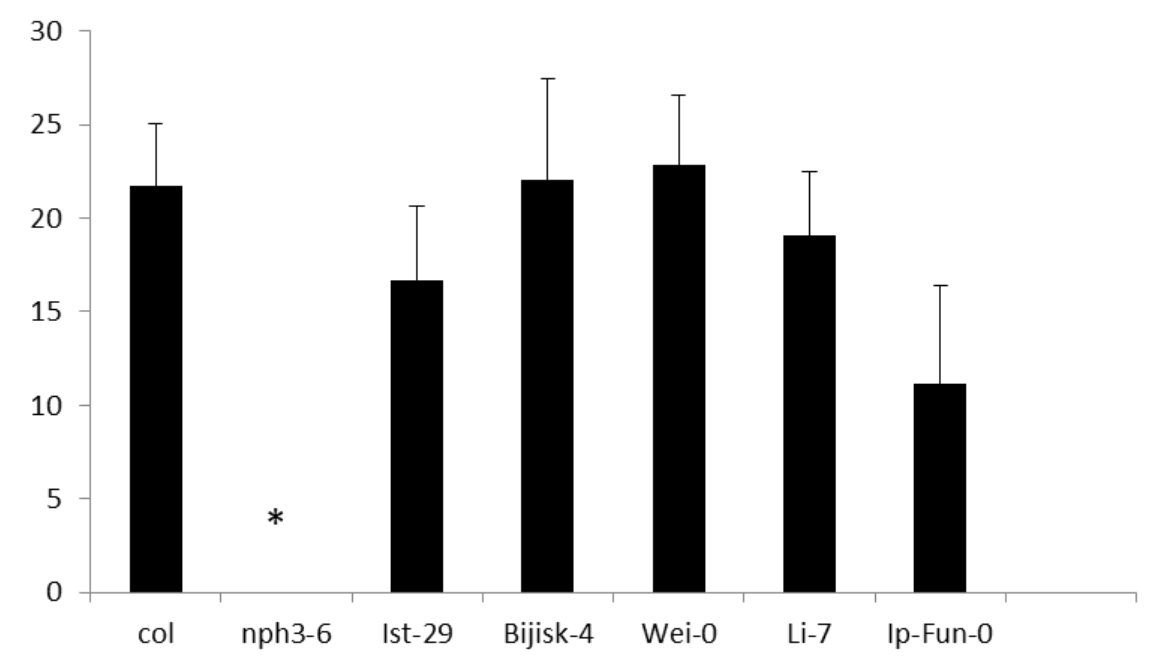

Figure 4-3 Preliminary hypocotyl phototropism of Arabidopsis accession lines at non-permissive temperature Hypocotyl phototropism at non-permissive temperature of $10^{\circ} \mathrm{C}$ of Arabidopsis thaliana ascension lines for 6 hours of unilateral blue light at a fluence rate of $0.1 \mu \mathrm{mol} \mathrm{m}{ }^{-2} \mathrm{~s}^{-1}$. The null nph3-6 mutant represents the negative control. Data is represented by average degrees of curvature with standard error denoted from one independent experiment. Statistical significance of mean average differing from that of Columbia is denoted with an asterisk $(P<0.05)$. 


\section{References}

Aggarwal, C., A.K. Banaś, A. Kasprowicz-Maluśki, C. Borghetti, J. Łabuz, J. Dobrucki, and H. Gabryś. 2014. Blue-light-activated phototropin2 trafficking from the cytoplasm to Golgi/post-Golgi vesicles. J. Exp. Bot. 65:3263-3276. doi:10.1093/jxb/eru172.

Ahmad, M., and A.R. Cashmore. 1993. HY4 gene of A. thaliana encodes a protein with characteristics of a blue-light photoreceptor. Nature. 366:162166. doi:10.1038/366162a0.

Ahmad, M., J.A. Jarillo, and A.R. Cashmore. 1998. Chimeric Proteins between cry1 and cry2 Arabidopsis Blue Light Photoreceptors Indicate Overlapping Functions and Varying Protein Stability. Plant Cell. 10:197-207.

Alonso-Blanco, C., J. Andrade, C. Becker, F. Bemm, J. Bergelson, K.M.M.

Borgwardt, J. Cao, E. Chae, T.M.M. Dezwaan, W. Ding, J.R.R. Ecker, M. Exposito-Alonso, A. Farlow, J. Fitz, X. Gan, D.G.G. Grimm, A.M.M. Hancock, S.R.R. Henz, S. Holm, M. Horton, M. Jarsulic, R.A.A. Kerstetter, A. Korte, P. Korte, C. Lanz, C.R. Lee, D. Meng, T.P.P. Michael, R. Mott, N.W.W. Muliyati, T. Nägele, M. Nagler, V. Nizhynska, M. Nordborg, P.Y.Y. Novikova, F.X. Picó, A. Platzer, F.A.A. Rabanal, A. Rodriguez, B.A.A. Rowan, P.A.A. Salomé, K.J.J. Schmid, R.J.J. Schmitz, Ü. Seren, F.G.G. Sperone, M. Sudkamp, H. Svardal, M.M.M. Tanzer, D. Todd, S.L.L. Volchenboum, C. Wang, G. Wang, X. Wang, W. Weckwerth, D. Weigel, and X. Zhou. 2016. 1,135 Genomes Reveal the Global Pattern of Polymorphism 
in Arabidopsis thaliana. Cell. 166:481-491. doi:10.1016/j.cell.2016.05.063.

Askinosie, S. 2016. Blue light- and ubiquitin-dependent influence on phototropin 1 abundance and movement at the plasma membrane. PhD Diss. Univ. Missouri.

Babourina, O., L. Godfrey, and K. Voltchanskii. 2004. Changes in ion fluxes during phototropic bending of etiolated oat coleoptiles. Ann. Bot. 94:187194. doi:10.1093/aob/mch126.

Bartolini, F., and G.G. Gundersen. 2006. Generation of noncentrosomal microtubule arrays. J. Cell Sci. 119:4155-4163. doi:10.1242/jcs.03227.

Baskin, T.I. 2001. On the alignment of cellulose microfibrils by cortical microtubules: A review and a model. Protoplasma. 215:150-171. doi:10.1007/BF01280311.

Baum, G., J.C. Long, G.I. Jenkins, and a J. Trewavas. 1999. Stimulation of the blue light phototropic receptor NPH1 causes a transient increase in cytosolic Ca2+. Proc. Natl. Acad. Sci. U. S. A. 96:13554-13559. doi:10.1073/pnas.96.23.13554.

Baxter, I., J. Tchieu, M.R. Sussman, M. Boutry, M.G. Palmgren, M. Gribskov, J.F. Harper, and K.B. Axelsen. 2003. Genomic Comparison of P-Type ATPase Ion Pumps in Arabidopsis and Rice. Plant Physiol. 132:618-628. doi:10.1104/pp.103.021923.

Ben-Aroya, S., X. Pan, J.D. Boeke, and P. Hieter. 2010. Making temperaturesensitive mutants. Methods Enzym. 470:181-204.

Benjamins, R., C.S. Galván Ampudia, P.J.J. Hooykaas, and R. Offringa. 2003. 
PINOID-Mediated Signaling Involves Calcium-Binding Proteins. Plant Physiol. 132:1623-1630. doi:10.1104/pp.103.019943.

Bennett, M.J., A. Marchant, H.G. Green, S.T. May, S.P. Ward, P.A. Millner, A.R. Walker, B. Schulz, and K.A. Feldmann. 1996. Arabidopsis AUX1 Gene : A Permease-Like Regulator of Root Gravitropism. Science. 273:948-950.

Bennett, S.R.M., J. Alvarez, G. Bossinger, and D.R. Smyth. 1995.

Morphogenesis in pinoid mutants of Arabidopsis thaliana. Plant J. 8:505520. doi:10.1046/j.1365-313X.1995.8040505.x.

Bennett, T. 2015. PIN proteins and the evolution of plant development. Trends Plant Sci. 20:498-507. doi:10.1016/j.tplants.2015.05.005.

Berben, G., M. Legrain, V. Gilliquet, and F. Hilger. 1990. The yeast regulatory gene PHO4 encodes a helix-loop-helix motif. Yeast. 6:451-454.

doi:10.1002/yea.320060510.

Blakeslee, J.J., A. Bandyopadhyay, W.A. Peer, S.N. Makam, and A.S. Murphy. 2004. Relocalization of the PIN1 auxin efflux facilitator plays a role in phototropic responses. Plant Physiol. 134:28-31. doi:10.1104/pp.103.031690.

Boer, D.R., A. Freire-Rios, W.A.M. Van Den Berg, T. Saaki, I.W. Manfield, S. Kepinski, I. López-Vidrieo, J.M. Franco-Zorrilla, S.C. De Vries, R. Solano, D. Weijers, and M. Coll. 2014. Structural basis for DNA binding specificity by the auxin-dependent ARF transcription factors. Cell. 156:577-589. doi:10.1016/j.cell.201312.027. Bögre, L., L. Ökrész, R. Henriques, and R.G. Anthony. 2003. Growth signalling 
pathways in Arabidopsis and the AGC protein kinases. Trends Plant Sci. 8:424-431. doi:10.1016/S1360-1385(03)00188-2.

Borner, G.H.H., K.S. Lilley, T.J. Stevens, and P. Dupree. 2003. Identification of Glycosylphosphatidylinositol-Anchored Proteins in Arabidopsis. A Proteomic and Genomic Analysis. Plant Physiol. 132:568-577. doi:10.1104/pp.103.021170.

Briggs, W.R., C.F. Beck, A.R. Cashmore, J.M. Christie, J. Hughes, J.A. Jarillo, T. Kagawa, H. Kanegae, E. Liscum, A. Nagatani, K. Okada, M. Salomon, W. Rüdiger, T. Sakai, M. Takano, M. Wada, and J.C. Watson. 2001. The Phototropin Family of Photoreceptors. Plant Cell. 13:993-997. doi:10.1007/s10265-002-0072-4.

Briggs, W.R., and E. Huala. 1999. Blue-Light Photoreceptors In Higher Plants. Annu. Rev. Cell Dev. Biol. 62:33-62.

de Carbonnel, M., P. Davis, M.R.G. Roelfsema, S. -i. Inoue, I. Schepens, P. Lariguet, M. Geisler, K. -i. Shimazaki, R. Hangarter, and C. Fankhauser. 2010. The Arabidopsis PHYTOCHROME KINASE SUBSTRATE2 Protein Is a Phototropin Signaling Element That Regulates Leaf Flattening and Leaf Positioning. Plant Physiol. 152:1391-1405. doi:10.1104/pp.109.150441. Carretero-Paulet, L., A. Galstyan, I. Roig-Villanova, J.F. Martinez-Garcia, J.R. Bilbao-Castro, and D.L. Robertson. 2010. Genome-Wide Classification and Evolutionary Analysis of the bHLH Family of Transcription Factors in Arabidopsis, Poplar, Rice, Moss, and Algae. Plant Physiol. 153:1398-1412. doi:10.1104/pp.110.153593. 
Chae, K., C.G. Isaacs, P.H. Reeves, G.S. Maloney, G.K. Muday, P. Nagpal, and J.W. Reed. 2012. Arabidopsis SMALL AUXIN UP RNA63 promotes hypocotyl and stamen filament elongation. Plant J. 71:684-697. doi:10.1111/j.1365-313X.2012.05024.x.

Chaves, I., R. Pokorny, M. Byrdin, N. Hoang, T. Ritz, K. Brettel, L.-O. Essen, G.T.J. van der Horst, A. Batschauer, and M. Ahmad. 2011. The Cryptochromes: Blue Light Photoreceptors in Plants and Animals. Annu. Rev. Plant Biol. 62:335-364. doi:10.1146/annurev-arplant-042110-103759. Cheng, Y., G. Qin, X. Dai, and Y. Zhao. 2007. NPY1, a BTB-NPH3-like protein, plays a critical role in auxin-regulated organogenesis in Arabidopsis. Proc. Natl. Acad. Sci. U. S. A. 104:18825-9. doi:10.1073/pnas.0708506104. Cheng, Y., G. Qin, X. Dai, and Y. Zhao. 2008. NPY genes and AGC kinases define two key steps in auxin-mediated organogenesis in Arabidopsis. Proc. Natl. Acad. Sci. U. S. A. 105:21017-21022. doi:10.1073/pnas.0809761106. Cho, M., S.H. Lee, and H.-T. Cho. 2007. P-Glycoprotein4 Displays Auxin Efflux Transporter-Like Action in Arabidopsis Root Hair Cells and Tobacco Cells. Plant Cell. 19:3930-3943. doi:10.1105/tpc.107.054288.

Christensen, S.K., N. Dagenais, J. Chory, and D. Weigel. 2000. Regulation of Auxin Response by the Protein Kinase PINOID. Cell. 100:469-478. doi:10.1016/S0092-8674(00)80682-0.

Christie, J., N. Suetsugu, S. Sullivan, and M. Wada. 2017. Shining Light on the Function of NPH3/RPT2-like Proteins in Phototropin Signalling. Plant Physiol. 176:1015-1024. doi:10.1104/pp.17.00835. 
Christie, J.M. 2007. Phototropin blue-light receptors. Annu. Rev. Plant Biol.

58:21-45. doi:10.1146/annurev.arplant.58.032806.103951.

Christie, J.M., L. Blackwood, J. Petersen, and S. Sullivan. 2015. Plant

flavoprotein photoreceptors. Plant Cell Physiol. 56:401-413.

doi:10.1093/pcp/pcu196.

Christie, J.M., P. Reymond, G.K. Powell, P. Bernasconi, A.A. Raibekas, E. Liscum, and W.R. Briggs. 1998. Arabidopsis NPH1: A Flavoprotein with the Properties of a Photoreceptor for Phototropism. Science 282:1698-1701. doi:10.1126/science.282.5394.1698.

Christie, J.M., M. Salomon, K. Nozue, M. Wada, and W.R. Briggs. 1999. LOV (light, oxygen, or voltage) domains of the blue-light photoreceptor phototropin (nph1): binding sites for the chromophore flavin mononucleotide. Proc. Natl. Acad. Sci. U. S. A. 96:8779-83.

Christie, J.M., H. Yang, G.L. Richter, S. Sullivan, C.E. Thomson, J. Lin, B.

Titapiwatanakun, M. Ennis, E. Kaiserli, O.R. Lee, J. Adamec, W.A. Peer, and A.S. Murphy. 2011. phot1 inhibition of ABCB19 primes lateral auxin fluxes in the shoot apex required for phototropism. PLoS Biol. 9:e1001076. doi:10.1371/journal.pbio.1001076.

Ciarbelli, A.R., A. Ciolfi, S. Salvucci, V. Ruzza, M. Possenti, M. Carabelli, A. Fruscalzo, G. Sessa, G. Morelli, and I. Ruberti. 2008. The Arabidopsis homeodomain-leucine zipper II gene family: diversity and redundancy. Plant Mol. Biol. 68:465-78. doi:10.1007/s11103-008-9383-8.

Clack, T., S. Mathews, and R.A. Sharrock. 1994. The phytochrome apoprotein 
family in Arabidopsis is encoded by five genes: the sequences and expression of PHYD and PHYE. Plant Mol. Biol. 25:413-427. doi:10.1007/BF00043870.

Clough, S.J., and A.F. Bent. 1998. Floral dip: a simplified method for Agrobacterium-mediated transformation of Arabidopsis thaliana. Plant $\mathrm{J}$. $16: 735-43$.

Cosgrove, D.J. 1997. Relaxation in a High-Stress Environment: The Molecular Bases of Extensible Cell Walls and Cell Enlargement. Plant Cell Online. 9:1031-1041. doi:10.1105/tpc.9.7.1031.

Cosgrove, D.J. 2000. Loosening of plant cell walls by expansins. Nature. 407: 321-326.

Crosson, S., S. Rajagopal, and K. Moffat. 2003. The LOV domain family: Photoresponsive signaling modules coupled to diverse output domains. Biochemistry. 42:2-10. doi:10.1021/bi026978l.

Curtis, M.D., and U. Grossniklaus. 2003. A Gateway Cloning Vector Set for HighThroughput Functional Analysis of Genes in Planta. Plant Physiol. 133:462469. doi:10.1104/pp.103.027979.specific.

DeBlasio, S.L., J.L. Mullen, D.R. Luesse, and R.P. Hangarter. 2003.

Phytochrome modulation of blue light-induced chloroplast movements in Arabidopsis. Plant Physiol. 133:1471-1479. doi:10.1104/pp.103.029116. Demarsy, E., I. Schepens, K. Okajima, M. Hersch, S. Bergmann, J. Christie, K.-I. Shimazaki, S. Tokutomi, and C. Fankhauser. 2012. Phytochrome Kinase Substrate 4 is phosphorylated by the phototropin 1 photoreceptor. EMBO J. 
31:3457-67. doi:10.1038/emboj.2012.186.

Deng, Z., J.A. Oses-Prieto, U. Kutschera, T.-S. Tseng, L. Hao, A.L. Burlingame, Z.-Y. Wang, and W.R. Briggs. 2014. Blue Light-Induced Proteomic Changes in Etiolated Arabidopsis Seedlings. J. Proteome Res. doi:10.1021/pr500010z.

Deshaies, R.J., and C.A.P. Joazeiro. 2009. RING domain E3 ubiquitin ligases. Annu. Rev. Biochem. 78:399-434.

doi:10.1146/annurev.biochem.78.101807.093809.

Dezfulian, M.H., E. Jalili, D.K.A. Roberto, B.L. Moss, K. Khoo, J.L. Nemhauser, and W.L. Crosby. 2016. Oligomerization of SCFTIR1 Is Essential for Aux/IAA Degradation and Auxin Signaling in Arabidopsis. PLoS Genet. 12:1-18. doi:10.1371/journal.pgen.1006301.

Ding, Z., C.S. Galván-Ampudia, E. Demarsy, Ł. Łangowski, J. Kleine-Vehn, Y. Fan, M.T. Morita, M. Tasaka, C. Fankhauser, R. Offringa, and J. Friml. 2011. Light-mediated polarization of the PIN3 auxin transporter for the phototropic response in Arabidopsis. Nat. Cell Biol. 13:447-453. doi:10.1038/ncb2208.

Doherty, G.J., and H.T. McMahon. 2009. Mechanisms of Endocytosis. Annu. Rev. Biochem. 78:857-902. doi:10.1146/annurev.biochem.78.081307.110540.

Dummer, M., C. Michalski, L.O. Essen, M. Rath, P. Galland, and C. Forreiter. 2016. EHB1 and AGD12, two calcium-dependent proteins affect gravitropism antagonistically in Arabidopsis thaliana. J. Plant Physiol. 206:114-124. doi:10.1016/j.jplph.2016.09.006. 
Esmon, C.A., A.G. Tinsley, K. Ljung, G. Sandberg, L.B. Hearne, and E. Liscum. 2006. A gradient of auxin and auxin-dependent transcription precedes tropic growth responses. Proc. Natl. Acad. Sci. U. S. A. 103:236-41. doi:10.1073/pnas.0507127103.

Fankhauser, C. 2001. The Phytochromes, a Family of Red/Far-red Absorbing Photoreceptors. J. Biol. Chem. 276:11453-11456. doi:10.1074/jbc.R100006200.

Fankhauser, C., K.-C. Yeh, J.C. Lagarias, H. Zhang, T.D. Elich, and J. Chory. 1999. PKS1, a Substrate Phosphorylated by Phytochrome That Modulates Light Signaling in Arabidopsis. Science 284:1539-1541. doi:10.1126/science.284.5419.1539.

Felle, H. 1988. Auxin causes oscillations of cytosolic free calcium and $\mathrm{pH} Z e a$ mays coleoptiles. Planta. 174:495-499. doi:10.1007/bf00634478.

Figueroa, P., G. Gusmaroli, G. Serino, J. Habashi, L. Ma, Y. Shen, S. Feng, M. Bostick, J. Callis, H. Hellmann, and X.W. Deng. 2005. Arabidopsis Has Two Redundant Cullin3 Proteins That Are Essential for Embryo Development and That Interact with RBX1 and BTB Proteins to Form Multisubunit E3 Ubiquitin Ligase Complexes in Vivo. Plant Cell. 17:1180-1195.

doi:10.1105/tpc.105.031989.

Folta, K.M., E.J. Lieg, T. Durham, and E.P. Spalding. 2003. Primary Inhibition of Hypocotyl Growth and Phototropisrn Depend Differently on PhototropinMediated Increases in Cytoplasmic Calcium Induced by Blue Light. 133:1464-1470. doi:10.1104/pp.103.024372.ways. 
Franklin, K.A., V.S. Larner, and C.C. Whitelam. 2005. The signal transducing photoreceptors of plants. Int. J. Dev. Biol. 49:653-664. doi:10.1387/ijdb.051989kf.

Franklin, K.A., and P.H. Quail. 2010. Phytochrome functions in Arabidopsis development. J. Exp. Bot. 61:11-24. doi:10.1093/jxb/erp304.

Friml, J. 2003. Auxin transport - shaping the plant. Curr. Opin. Plant Biol. 6:7-12. doi:10.1016/S1369-5266(02)00003-1.

Friml, J., J. Wiśniewska, E. Benková, K. Mendgen, and K. Palme. 2002. Lateral relocation of auxin efflux regulator PIN3 mediates tropism in Arabidopsis. Nature. 415:806-809. doi:10.1038/415806a.

Friml, J., X. Yang, M. Michniewicz, D. Weijers, A. Quint, O. Tietz, R. Benjamins, P.B.F. Ouwerkerk, K. Ljung, G. Sandberg, P.J.J. Hooykaas, K. Palme, and R. Offringa. 2004. A PINOID-dependent binary switch in apical-basal PIN polar targeting directs auxin efflux. Science 306:862-865.

doi:10.1126/science.1100618.

Furutani, M., T. Kajiwara, T. Kato, B.S. Treml, C. Stockum, R.A. Torres-Ruiz, and M. Tasaka. 2007. The gene MACCHI-BOU 4/ENHANCER OF PINOID encodes a NPH3-like protein and reveals similarities between organogenesis and phototropism at the molecular level. Development. 134:3849-3859. doi:10.1242/dev.009654.

Gehring, C., D. Williams, S. Cody, and R. Parish. 1990. Phototropism and geotropism in maize coleoptiles are spatially correlated with increases in cytosolic free calcium. Nature. 345:528-30. doi:10.1038/345528a0. 
Geldner, N., S. Richter, A. Vieten, S. Marquardt, R. A. Torres-Ruiz, U. Mayer, G. Jurgens. 2003. Partial loss-of-function alleles reveal a role for GNOM in auxin transport-related, post-embryonic development of Arabidopsis. Development. 131:389-400. doi:10.1242/dev.00926.

Geldner, N., N. Anders, H. Wolters, J. Keicher, W. Kornberger, P. Muller, A. Delbarre, T. Ueda, A. Nakano, and G. Jürgens. 2003. The Arabidopsis GNOM ARF-GEF mediates endosomal recycling, auxin transport, and auxindependent plant growth. Cell. 112:219-230. doi:10.1016/S00928674(03)00003-5.

Genschik, P., I. Sumara, and E. Lechner. 2013. The emerging family of CULLIN3-RING ubiquitin ligases (CRL3s): cellular functions and disease implications. EMBO J. 32:2307-20. doi:10.1038/emboj.2013.173.

Gilkerson, J., D.R. Kelley, R. Tam, M. Estelle, and J. Callis. 2015. Lysine Residues Are Not Required for Proteasome-Mediated Proteolysis of the Auxin/Indole Acidic Acid Protein IAA1. Plant Physiol. 168:708-720. doi:10.1104/pp.15.00402.

Grones, P., and J. Friml. 2015. Auxin transporters and binding proteins at a glance. J. Cell Sci. 128:1-7. doi:10.1242/jcs.159418.

Grunewald, W., and J. Friml. 2010. The march of the PINs: developmental plasticity by dynamic polar targeting in plant cells. EMBO J. 29:2700-14. doi:10.1038/emboj.2010.181.

Guilfoyle, T.J. 2015. The PB1 Domain in Auxin Response Factor and Aux/IAA Proteins: A Versatile Protein Interaction Module in the Auxin Response. 
Plant Cell Online. 27:33-43. doi:10.1105/tpc.114.132753.

Ha, C.M., J.H. Jun, and J.C. Fletcher. 2010. Shoot apical meristem form and function. Curr. Top. Dev. Biol. 91:103-140. doi:10.1016/S00702153(10)91004-1.

Haga, K., M. Takano, R. Neumann, and M. lino. 2005. The Rice COLEOPTILE PHOTOTROPISM1 Gene Encoding an Ortholog of Arabidopsis NPH3 Is Required for Phototropism of Coleoptiles and Lateral Translocation of Auxin. Plant Cell. 17:103-115. doi:10.1105/tpc.104.028357.

Haga, K., T. Tsuchida-Mayama, M. Yamada, and T. Sakai. 2015. Arabidopsis ROOT PHOTOTROPISM2 Contributes to the Adaptation to High-Intensity Light in Phototropic Responses. Plant Cell. 27:1098-1112. doi:10.1105/tpc.15.00178.

Hagen, G., and T. Guilfoyle. 2002. Auxin-responsive gene expression: genes, promoters and regulatory factors. Plant Mol. Biol. 49:373-85.

Haglund, K., and I. Dikic. 2005. Ubiquitylation and cell signaling. EMBO J. 24:3353-9. doi:10.1038/sj.emboj.7600808.

Han, I.-S., T.-S. Tseng, W. Eisinger, and W.R. Briggs. 2008. Phytochrome A Regulates the Intracellular Distribution of Phototropin 1-Green Fluorescent Protein in Arabidopsis thaliana. Plant Cell Online. 20:2835-2847. doi:10.1105/tpc.108.059915.

Han, I.S., H.Y. Cho, A. Moni, A.Y. Lee, and W.R. Briggs. 2013. Investigations on the photoregulation of chloroplast movement and leaf positioning in arabidopsis. Plant Cell Physiol. 54:48-56. doi:10.1093/pcp/pcs098. 
Han, M., Y. Park, I. Kim, E.-H. Kim, T.-K. Yu, S. Rhee, and J.-Y. Suh. 2014. Structural basis for the auxin-induced transcriptional regulation by Aux/IAA17. Proc. Natl. Acad. Sci. 111:18613-18618. doi:10.1073/pnas.1419525112.

Harada, A., T. Sakai, and K. Okada. 2003. phot1 and phot2 mediate blue lightinduced transient increases in cytosolic Ca2+ differently in Arabidopsis leaves. Proc. Natl. Acad. Sci. 100:8583-8588.

doi:10.1073/pnas.1336802100.

Harada, A., and K. Shimazaki. 2007. Phototropins and blue light-dependent calcium signaling in higher plants. Photochem. Photobiol. 83:102-11. doi:10.1562/2006-03-08-IR-837.

Harada, A., A. Takemiya, S.I. Inoue, T. Sakai, and K.I. Shimazaki. 2013. Role of RPT2 in leaf positioning and flattening and a possible inhibition of phot2 signaling by phot1. Plant Cell Physiol. 54:36-47. doi:10.1093/pcp/pcs094.

Harper, R.M., E.L. Stowe-Evans, D.R. Luesse, H. Muto, K. Tatematsu, M.K. Watahiki, K. Yamamoto, and E. Liscum. 2000. The NPH4 locus encodes the auxin response factor ARF7, a conditional regulator of differential growth in aerial Arabidopsis tissue. Plant Cell. 12:757-70.

Harper, S.M., J.M. Christie, and K.H. Gardner. 2004. Disruption of the LOV-Ja helix interaction activates phototropin kinase activity. Biochemistry. 43:16184-16192. doi:10.1021/bi048092i.

Haruta, M., H.L. Burch, R.B. Nelson, G. Barrett-Wilt, K.G. Kline, S.B. Mohsin, J.C. Young, M.S. Otegui, and M.R. Sussman. 2010. Molecular 
characterization of mutant Arabidopsis plants with reduced plasma membrane proton pump activity. J. Biol. Chem. 285:17918-17929. doi:10.1074/jbc.M110.101733.

Holland, J.J., D. Roberts, and E. Liscum. 2009. Understanding phototropism: from Darwin to today. J. Exp. Bot. 60:1969-78. doi:10.1093/jxb/erp113. Horowitz, N.H. 1950. Biochemical Genetics of Neurospora. Adv. Genet. 33-71. Horowitz, N.H., and H.K. Mitchell. 1950. BIOCHEMICAL GENETICS. Annu. Rev. Biochem. 465-486.

Hotton, S.K., and J. Callis. 2008. Regulation of cullin RING ligases. Annu. Rev. Plant Biol. 59:467-89. doi:10.1146/annurev.arplant.58.032806.104011. Huala, E., P.W. Oeller, E. Liscum, I.-S.S. Han, E. Larsen, and W.R. Briggs. 1997. Arabidopsis NPH1: A Protein Kinase with a Putative Redox-Sensing Domain. Science 278:2120-2123. doi:10.1126/science.278.5346.2120. Huang, F., M. Kemel Zago, L. Abas, A. van Marion, C.S. Galván-Ampudia, and R. Offringa. 2010. Phosphorylation of Conserved PIN Motifs Directs Arabidopsis PIN1 Polarity and Auxin Transport. Plant Cell. 22:1129-1142. doi:10.1105/tpc.109.072678.

Hughes, J. 2013. Phytochrome cytoplasmic signaling. Annu. Rev. Plant Biol. 64:377-402. doi:10.1146/annurev-arplant-050312-120045. Inada, S., M. Ohgishi, T. Mayama, K. Okada, and T. Sakai. 2004. RPT2 Is a Signal Transducer Involved in Phototropic Response and Stomatal Opening by Association with Phototropin 1 in Arabidopsis thaliana. Plant Cell. 16:887-896. doi:10.1105/tpc.019901. 
Inoue, S.-I., T. Kinoshita, M. Matsumoto, K.I. Nakayama, M. Doi, and K.-I. Shimazaki. 2008a. Blue light-induced autophosphorylation of phototropin is a primary step for signaling. Proc. Natl. Acad. Sci. U. S. A. 105:5626-31. doi:10.1073/pnas.0709189105.

Inoue, S., T. Matsushita, Y. Tomokiyo, M. Matsumoto, K.I. Nakayama, T. Kinoshita, and K. Shimazaki. 2011. Functional analyses of the activation loop of phototropin2 in Arabidopsis. Plant Physiol. 156:117-28. doi:10.1104/pp.111.175943.

Inoue, S.I., T. Kinoshita, A. Takemiya, M. Doi, and K.I. Shimazaki. 2008b. Leaf positioning of Arabidopsis in response to blue light. Mol. Plant. 1:15-26. doi:10.1093/mp/ssm001.

Jaedicke, K., A.L. Lichtenthäler, R. Meyberg, M. Zeidler, and J. Hughes. 2012. A phytochrome-phototropin light signaling complex at the plasma membrane. Proc. Natl. Acad. Sci. 109:12231-12236. doi:10.1073/pnas.1120203109. Jain, M., A.K. Tyagi, and J.P. Khurana. 2006. Genome-wide analysis, evolutionary expansion, and expression of early auxin-responsive SAUR gene family in rice (Oryza sativa). Genomics. 88:360-371. doi:10.1016/j.ygeno.2006.04.008.

Janoudi A-K, R. Konjevic, P. Apel, and K.L. Poff. 1992. Time threshold for second positive phototropism is decreased by a preirradiation with red light. Plant Physiol. 99:1422-1425. doi:10.1104/pp.99.4.1422.

Janoudi, A., W.R. Gordon, D. Wagner, P. Quail, and K.L. Poff. 1997. Multiple Phytochromes Are Involved in Red-Light-Induced Enhancement of First- 
Positive Phototropism in Arabidopsis thaliana. Plant Physiol. 113:975-979. doi:10.1104/pp.113.3.975.

Janoudi, A., and K.L. Poff. 1993. Desensitization and Recovery of Phototropic Re s p o n s i ve n e ss i n Arabidopsis thaliana. Plant Physiol. 1175-1180. Jarillo, J.A., H. Gabrys, J. Capel, J.M. Alonso, J.R. Ecker, and A.R. Cashmore. 2001. Phototropin-related NPL1 controls chloroplast relocation induced by blue light. Nature. 410:952-954. doi:10.1038/35073622.

Johansson, H., and M. Zeidler. 2016. Automatic Chloroplast Movement Analysis. In In: Duque P. (eds) Environmental Responses in Plants. Humana Press, New York, NY. 29-35.

Jones, M.A., K.A. Feeney, S.M. Kelly, and J.M. Christie. 2007. Mutational analysis of phototropin 1 provides insights into the mechanism underlying LOV2 signal transmission. J. Biol. Chem. 282:6405-6414. doi:10.1074/jbc.M605969200.

Kagawa, T., T. Sakai, N. Suetsugu, K. Oikawa, S. Ishiguro, T. Kato, S. Tabata, K. Okada, and M. Wada. 2001. Arabidopsis NPL1: A phototropin homolog controlling the chloroplast high-light avoidance response. Science 291:2138-2141. doi:10.1126/science.291.5511.2138.

Kaiserli, E., S. Sullivan, M. A Jones, K. A Feeney, and J.M. Christie. 2009. Domain swapping to assess the mechanistic basis of Arabidopsis phototropin 1 receptor kinase activation and endocytosis by blue light. Plant Cell. 21:3226-44. doi:10.1105/tpc.109.067876.

Kami, C., L. Allenbach, M. Zourelidou, K. Ljung, F. Schütz, E. Isono, M.K. 
Watahiki, K.T. Yamamoto, C. Schwechheimer, and C. Fankhauser. 2014. Reduced phototropism in pks mutants may be due to altered auxin-regulated gene expression or reduced lateral auxin transport. Plant J. 77:393-403. doi:10.1111/tpj.12395.

Kami, C., M. Hersch, M. Trevisan, T. Genoud, A. Hiltbrunner, S. Bergmann, and C. Fankhauser. 2012. Nuclear Phytochrome A Signaling Promotes Phototropism in Arabidopsis. Plant Cell. 24:566-576. doi:10.1105/tpc.111.095083.

Kami, C., S. Lorrain, P. Hornitschek, and C. Fankhauser. 2010. Light-regulated plant growth and development. Current topics in developmental biology. 91 . 29-66.

Kansup, J., D. Tsugama, S. Liu, and T. Takano. 2014. Arabidopsis G-protein $\beta$ subunit AGB1 interacts with NPH3 and is involved in phototropism. Biochem. Biophys. Res. Commun. 445:54-57. doi:10.1016/j.bbrc.2014.01.106.

Khurana, J.P., and K.L. Poff. 1989. Mutants of Arabidopsis thaliana with altered phototropism. Planta. 178:400-406.

Kim, J., K. Harter, and A. Theologis. 1997. Protein-protein interactions among the Aux/IAA proteins. Proc. Natl. Acad. Sci. U. S. A. 94:11786-91. doi:10.1073/pnas.94.22.11786.

Kinoshita, T., M. Doi, and N. Suetsugu. 2001. Regulation of Stomatal Opening. Nature. 414:0-4.

Knauer, T., M. Dummer, F. Landgraf, and C. Forreiter. 2011. A Negative Effector of Blue Light-Induced and Gravitropic Bending in Arabidopsis. Plant Physiol. 
156:439-447. doi:10.1104/pp.110.167411.

Kong, S.-G., T. Suzuki, K. Tamura, N. Mochizuki, I. Hara-Nishimura, and A. Nagatani. 2006. Blue light-induced association of phototropin 2 with the Golgi apparatus. Plant J. 45:994-1005. doi:10.1111/j.1365313X.2006.02667.x.

Kong, S.G., T. Kagawa, M. Wada, and A. Nagatani. 2013a. A C-terminal membrane association domain of phototropin 2 is necessary for chloroplast movement. Plant Cell Physiol. 54:57-68. doi:10.1093/pcp/pcs132.

Kong, S.G., T. Kinoshita, K.I. Shimazaki, N. Mochizuki, T. Suzuki, and A. Nagatani. 2007. The C-terminal kinase fragment of Arabidopsis phototropin 2 triggers constitutive phototropin responses. Plant J. 51:862-873. doi:10.1111/j.1365-313X.2007.03187.x.

Kong, S.G., N. Suetsugu, S. Kikuchi, M. Nakai, A. Nagatani, and M. Wada. 2013b. Both phototropin 1 and 2 localize on the chloroplast outer membrane with distinct localization activity. Plant Cell Physiol. 54:80-92. doi:10.1093/pcp/pcs151.

Kong, S.G., and M. Wada. 2016. Molecular basis of chloroplast photorelocation movement. J. Plant Res. 129:159-166. doi:10.1007/s10265-016-0788-1. Konieczny, A., and F.M. Ausubel. 1993. A procedure for mapping Arabidopsis mutations using co-dominant ecotype-specific PCR-based markers. Plant J. 4:403-410.

Korasick, D.A., C.S. Westfall, S.G. Lee, M.H. Nanao, R. Dumas, G. Hagen, T.J. Guilfoyle, J.M. Jez, and L.C. Strader. 2014. Molecular basis for AUXIN 
RESPONSE FACTOR protein interaction and the control of auxin response repression. Proc. Natl. Acad. Sci. 111:5427-5432.

doi:10.1073/pnas.1400074111.

Kozuka, T., N. Suetsugu, M. Wada, and A. Nagatani. 2013. Antagonistic regulation of leaf flattening by phytochrome $B$ and phototropin in Arabidopsis thaliana. Plant Cell Physiol. 54:69-79. doi:10.1093/pcp/pcs134.

Lalanne, E., C. Michaelidis, J.M. Moore, W. Gagliano, A. Johnson, R. Patel, R. Howden, J.P. Vielle-Calzada, U. Grossniklaus, and D. Twell. 2004. Analysis of transposon insertion mutants highlights the diversity of mechanisms underlying male progamic development in Arabidopsis. Genetics. 167:19751986. doi:10.1534/genetics.104.030270.

Lariguet, P., H.E. Boccalandro, J.M. Alonso, J.R. Ecker, J. Chory, J.J. Casal, and C. Fankhauser. 2003. A Growth Regulatory Loop That Provides Homeostasis to Phytochrome A Signaling. Plant Cell. 15:2966-2978. doi:10.1105/tpc.014563.2.

Lariguet, P., and C. Dunand. 2005. Plant photoreceptors: Phylogenetic overview. J. Mol. Evol. 61:559-569. doi:10.1007/s00239-004-0294-2.

Lariguet, P., and C. Fankhauser. 2004. Hypocotyl growth orientation in blue light is determined by phytochrome A inhibition of gravitropism and phototropin promotion of phototropism. Plant J. 40:826-834. doi:10.1111/j.1365313X.2004.02256.x.

Lariguet, P., I. Schepens, D. Hodgson, U. V Pedmale, M. Trevisan, C. Kami, M. de Carbonnel, J.M. Alonso, J.R. Ecker, E. Liscum, and C. Fankhauser. 
2006. PHYTOCHROME KINASE SUBSTRATE 1 is a phototropin 1 binding protein required for phototropism. Proc. Natl. Acad. Sci. U. S. A. 103:101349. doi:10.1073/pnas.0603799103.

Lascève, G., J. Leymarie, M.A. Olney, E. Liscum, J.M. Christie, A. Vavasseur, and W.R. Briggs. 1999. Arabidopsis contains at least four independent bluelight-activated signal transduction pathways. Plant Physiol. 120:605-614. doi:10.1104/pp.120.2.605.

Lavy, M., and M. Estelle. 2016. Mechanisms of auxin signaling. Development. 143:3226-3229. doi:10.1242/dev.131870.

Lee, S., S. Lee, K.Y. Yang, Y.M. Kim, S.Y. Park, S.Y. Kim, and M.S. Soh. 2006. Overexpression of PRE1 and its homologous genes activates gibberellindependent responses in Arabidopsis thaliana. Plant Cell Physiol. 47:591600. doi:10.1093/pcp/pcj026.

Lewis, D.R., N.D. Miller, B.L. Splitt, G. Wu, and E.P. Spalding. 2007. Separating the Roles of Acropetal and Basipetal Auxin Transport on Gravitropism with Mutations in Two Arabidopsis Multidrug Resistance-Like ABC Transporter Genes. Plant Cell. 19:1838-1850. doi:10.1105/tpc.107.051599.

Li, A.C., and D.J. Cosgrove. 2001. Grass group I pollen allergens ( $\beta$-expansins) lack proteinase activity and do not cause wall loosening via proteolysis. Eur. J. Biochem. 268:4217-4226. doi:10.1046/j.1432-1327.2001.02336.x.

Li, F.-W., C.J. Rothfels, M. Melkonian, J.C. Villarreal, D.W. Stevenson, S.W. Graham, G.K.-S. Wong, S. Mathews, and K.M. Pryer. 2015. The origin and evolution of phototropins. Front. Plant Sci. 6:1-11. 
doi:10.3389/fpls.2015.00637.

Li, X., X. Duan, H. Jiang, Y. Sun, Y. Tang, Z. Yuan, and J. Guo. 2006. GenomeWide Analysis of Basic / Helix-Loop-Helix Transcription Factor Family in Rice and Arabidopsis. Plant Physiol. 141:1167-1184. doi:10.1104/pp.106.080580.2001.

Li, Y., X. Dai, Y. Cheng, and Y. Zhao. 2011. NPY genes play an essential role in root gravitropic responses in Arabidopsis. Mol. Plant. 4:171-179. doi:10.1093/mp/ssq052.

Li, Y., C.P. Darley, V. Ongaro, A. Fleming, O. Schipper, S.L. Baldauf, and S.J. McQueen-Mason. 2002. Plant Expansins Are a Complex Multigene Family with an Ancient Evolutionary Origin. Plant Physiol. 128:854-864. doi:10.1104/pp.010658.

Lindeboom, J.J., M. Nakamura, A. Hibbel, K. Shundyak, R. Gutierrez, T. Ketelaar, A.M.C. Emons, B.M. Mulder, V. Kirik, and D.W. Ehrhardt. 2013. A mechanism for reorientation of cortical microtubule arrays driven by microtubule severing. Science. 342:1245533. doi:10.1126/science.1245533.

Liscum, E. 2016. Blue Light-Induced Intracellular Movement of Phototropins: Functional Relevance or Red Herring? Front. Plant Sci. 7:1-5. doi:10.3389/fpls.2016.00827.

Liscum, E., S.K. Askinosie, D.L. Leuchtman, J. Morrow, K.T. Willenburg, and D.R. Coats. 2014. Phototropism: Growing towards an understanding of plant movement. Plant Cell. 26. doi:10.1105/tpc.113.119727.

Liscum, E., and W.R. Briggs. 1995. Mutations in the NPH1 locus of Arabidopsis 
disrupt the perception of phototropic stimuli. Plant Cell. 7:473-85. doi:10.1105/tpc.7.4.473.

Liscum, E., and W.R. Briggs. 1996. Mutations of Arabidopsis in potential transduction and response components of the phototropic signaling pathway. Plant Physiol. 112:291-6.

Liscum, E., and J.W. Reed. 2002. Genetics of Aux/IAA and ARF action in plant growth and development. Plant Mol. Biol. 49:387-400.

Liu, B., Z. Yang, A. Gomez, B. Liu, C. Lin, and Y. Oka. 2016. Signaling mechanisms of plant cryptochromes in Arabidopsis thaliana. J. Plant Res. 129:137-148. doi:10.1007/s10265-015-0782-z.

Liu, B., Z. Zuo, H. Liu, X. Liu, and C. Lin. 2011. Arabidopsis cryptochrome 1 interacts with SPA1 to suppress COP1 activity in response to blue light. Genes Dev. 25:1029-1034. doi:10.1101/gad.2025011.

Liu, H., B. Liu, C. Zhao, M. Pepper, and C. Lin. 2012. The action mechanisms of plant cryptochromes. Trends Plant Sci. 16:684-691. doi:10.1016/j.tplants.2011.09.002.The.

Ludwig, S.R., L.F. Habera, S.L. Dellaporta, and S.R. Wessler. 1989. Lc, a member of the maize $\mathrm{R}$ gene family responsible for tissue-specific anthocyanin production, encodes a protein similar to transcriptional activators and contains the myc-homology region. Proc. Natl. Acad. Sci. U. S. A. 86:7092-7096. doi:10.1073/pnas.86.18.7092.

Mao, K., Q. Dong, C. Li, C. Liu, and F. Ma. 2017. Genome Wide Identification and Characterization of Apple bHLH Transcription Factors and Expression 
Analysis in Response to Drought and Salt Stress. Front. Plant Sci. 8:480. doi:10.3389/fpls.2017.00480.

Mara, C.D., T. Huang, and V.F. Irish. 2010. The Arabidopsis Floral Homeotic Proteins APETALA3 and PISTILLATA Negatively Regulate the BANQUO Genes Implicated in Light Signaling. Plant Cell. 22:690-702. doi:10.1105/tpc.109.065946.

Marchler-Bauer, A., C. Zheng, F. Chitsaz, M.K. Derbyshire, L.Y. Geer, R.C. Geer, N.R. Gonzales, M. Gwadz, D.I. Hurwitz, C.J. Lanczycki, F. Lu, S. Lu, G.H. Marchler, J.S. Song, N. Thanki, R.A. Yamashita, D. Zhang, and S.H. Bryant. 2013. CDD: Conserved domains and protein three-dimensional structure. Nucleic Acids Res. 41:348-352. doi:10.1093/nar/gks1243.

McCallum, C.M., L. Comai, E. a Greene, and S. Henikoff. 2000. Targeting induced local lesions in genomes (TILLING) for plant functional genomics. Plant Physiol. 123:439-42.

McClure, B.A., and T. Guilfoyle. 1987. Characterization of a class of small auxininducible soybean polyadenylated RNAs. Plant Mol. Biol. 9:611-623. doi:10.1007/BF00020537.

McDowell, G.S., and A. Philpott. 2013. Non-canonical ubiquitylation: Mechanisms and consequences. Int. J. Biochem. Cell Biol. 45:1833-1842. doi:10.1016/j.biocel.2013.05.026.

Mcqueen-mason, S., D.M. Durachko, and D.J. Cosgrove. 1992. Two Endogenous Proteins That Induce Cell Wall Extension in Plants. Plant Cell. 4:1425-1433. doi:10.1105/tpc.4.11.1425. 
McSteen, P. 2010. Auxin and monocot development. Cold Spring Harb.

Perspect. Biol. 2:a001479. doi:10.1101/cshperspect.a001479.

Michalski, C., M. Dümmer, P. Galland, and C. Forreiter. 2017. Impact of EHB1 and AGD12 on Root and Hypocotyl Phototropism in Arabidopsis thaliana. J. Plant Growth Regul. 36:660-668. doi:10.1007/s00344-017-9667-9.

Møller, S.G., P.J. Ingles, and G.C. Whitelam. 2002. The Cell Biology of phytochrome signaling. New Phytol. 154:553-590. doi:10.1046/j.14698137.2002.00419.x.

Motchoulski, A., and E. Liscum. 1999. Arabidopsis NPH3: A NPH1 Photoreceptor-Interacting Protein Essential for Phototropism. Science 286:961-964. doi:10.1126/science.286.5441.961.

Murre, C., P.S. McCaw, and D. Baltimore. 1989. A new DNA binding and dimerization motif in immunoglobulin enhancer binding, daughterless, MyoD, and myc proteins. Cell. 56:777-783. doi:10.1016/0092-8674(89)90682-X.

Nagashima, A., G. Suzuki, Y. Uehara, K. Saji, T. Furukawa, T. Koshiba, M. Sekimoto, S. Fujioka, T. Kuroha, M. Kojima, H. Sakakibara, N. Fujisawa, K. Okada, and T. Sakai. 2008. Phytochromes and cryptochromes regulate the differential growth of Arabidopsis hypocotyls in both a PGP19-dependent and a PGP19-independent manner. Plant J. 53:516-529.

doi:10.1111/j.1365-313X.2007.03358.x.

Nakasako, M., K. Zikihara, D. Matsuoka, H. Katsura, and S. Tokutomi. 2008. Structural Basis of the LOV1 Dimerization of Arabidopsis Phototropins 1 and 2. J. Mol. Biol. 381:718-733. doi:10.1016/j.jmb.2008.06.033. 
Nakazawa, M., N. Yabe, T. Ichikawa, Y.Y. Yamamoto, T. Yoshizumi, K. Hasunuma, and M. Matsui. 2001. DFL1, an auxin-responsive GH3 gene homologue, negatively regulates shoot cell elongation and lateral root formation, and positively regulates the light response of hypocotyl length. Plant J. 25:213-221. doi:10.1046/j.1365-313X.2001.00957.x.

Ni, M., J.M. Tepperman, and P.H. Quail. 1998. PIF3, a phytochrome-interacting factor necessary for normal photoinduced signal transduction, is a novel basic helix-loop-helix protein. Cell. 95:657-667. doi:10.1016/S00928674(00)81636-0.

Nick, P., R. Bergfeld, E. Schäfer, and P. Schopfer. 1990. Unilateral reorientation of microtubules at the outer epidermal wall during photo- and gravitropic curvature of maize coleoptiles and sunflower hypocotyls. Planta. 181:162168. doi:10.1007/BF02411533.

Noh, B., A. Bandyopadhyay, W.A. Peer, E.P. Spalding, and A.S. Murphy. 2003. Enhanced gravi-and photropism in plant mislocalizing the auxin efflux protein PIN1. Nature. 423:999-1002. doi:10.1038/nature01696.1.

Nozue, K., T. Kanegae, T. Imaizumi, S. Fukuda, H. Okamoto, K.C. Yeh, J.C. Lagarias, and M. Wada. 1998. A phytochrome from the fern Adiantum with features of the putative photoreceptor NPH1. Proc. Natl. Acad. Sci. U. S. A. 95:15826-15830. doi:10.1073/pnas.95.26.15826.

Ohgishi, M., K. Saji, K. Okada, and T. Sakai. 2004. Functional analysis of each blue light receptor, cry1, cry2, phot1, and phot2, by using combinatorial multiple mutants in Arabidopsis. Proc. Natl. Acad. Sci. USA. 101:2223-2228. 
Okada, K., J. Ueda, M.K. Komaki, C.J. Bell, and Y. Shimura. 1991. Requirement of the Auxin Polar Transport System in Early Stages of Arabidopsis Floral Bud Formation. Plant Cell. 3:677-684. doi:10.1105/tpc.3.7.677.

Okada, K., and Y. Shimura. 1992. Aspects of recent developments in mutational studies of plant signaling pathways. Cell. 70:369-372. doi:10.1016/00928674(92)90159-A.

Okada, K., and Y. Shimura. 1992. Mutational Analysis of Root Gravitropism and Phototropism of Arabidopsis thaliana Seedlings. Aust. J. Plant Physiol. 19:439-48. doi:10.1071/PP9920439.

Okushima, Y., P.J. Overvoorde, K. Arima, J.M. Alonso, A. Chan, C. Chang, J.R. Ecker, B. Hughes, A. Lui, D. Nguyen, C. Onodera, H. Quach, and A. Smith. 2005. Functional Genomic Analysis of the AUXIN RESPONSE FACTOR Gene Family Members in Arabidopsis thaliana. Plant Cell. 17:444-463. doi:10.1105/tpc.104.028316.2.

Paredez, A.R., C.R. Somerville, and D.W. Ehrhardt. 2006. Visualization of cellulose synthase demonstrates functional association with microtubules. Science 312:1491-1495. doi:10.1126/science.1126551.

Parihar, P., R. Singh, S. Singh, D.K. Tripathi, D.K. Chauhan, V.P. Singh, and S.M. Prasad. 2016. Photoreceptors mapping from past history till date. J. Photochem. Photobiol. B Biol. 162:223-231. doi:10.1016/j.jphotobiol.2016.06.020.

Park, J.E., P.J. Seo, A.K. Lee, J.H. Jung, Y.S. Kim, and C.M. Park. 2007. An Arabidopsis GH3 gene, encoding an auxin-conjugating enzyme, mediates 
phytochrome B-regulated light signals in hypocotyl growth. Plant Cell Physiol. 48:1236-1241. doi:10.1093/pcp/pcm086.

Park, Y.B., and D.J. Cosgrove. 2012. A Revised Architecture of Primary Cell Walls Based on Biomechanical Changes Induced by Substrate-Specific Endoglucanases. Plant Physiol. 158:1933-1943. doi:10.1104/pp.111.192880.

Parks, B.M., P.H. Quail, and R.P. Hangarter. 1996. Phytochrome A regulates red-light induction of phototropic enhancement in Arabidopsis. Plant Physiol. 110:155-162. doi:10.1104/pp.110.1.155.

Parry, G., A. Delbarre, A. Marchant, R. Swarup, R. Napier, C. PerrotRechenmann, and M.J. Bennett. 2001. Novel auxin transport inhibitors phenocopy the auxin influx carrier mutation aux1. Plant J. 25:399-406. doi:10.1046/j.1365-313X.2001.00970.x.

Pedmale, U. V. 2008. Role of nonphototropic hypocotyl 3 (nph3) in regulation of phototropism in ARABIDOPSIS. PhD Diss. Univ. Missouri.

Pedmale, U. V, R.B. Celaya, and E. Liscum. 2010. Phototropism : Mechanism and Outcomes. Arab. B. 1-26. doi:10.1199/tab.0042.

Pedmale, U. V, and E. Liscum. 2007. Regulation of phototropic signaling in Arabidopsis via phosphorylation state changes in the phototropin 1interacting protein NPH3. J. Biol. Chem. 282:19992-20001. doi:10.1074/jbc.M702551200.

Peer, W.A., J.J. Blakeslee, H. Yang, and A.S. Murphy. 2011. Seven things we think we know about auxin transport. Mol. Plant. 4:487-504. 
doi:10.1093/mp/ssr034.

Petricka, J.J., N.K. Clay, and T.M. Nelson. 2008. Vein patterning screens and the defectively organized tributaries mutants in Arabidopsis thaliana. Plant $\mathrm{J}$. 56:251-263. doi:10.1111/j.1365-313X.2008.03595.x.

Pires, N., and L. Dolan. 2010. Origin and diversification of basic-helix-loop-helix proteins in plants. Mol. Biol. Evol. 27:862-874. doi:10.1093/molbev/msp288.

Preuten, T., L. Blackwood, J.M. Christie, and C. Fankhauser. 2015. Lipid anchoring of arabidopsis phototropin 1 to assess the functional significance of receptor internalization: Should I stay or should I go? New Phytol. 206:1038-1050. doi:10.1111/nph.13299.

Qiu, T., Y. Chen, M. Li, Y. Kong, Y. Zhu, N. Han, H. Bian, M. Zhu, and J. Wang. 2013. The tissue-specific and developmentally regulated expression patterns of the SAUR41 subfamily of SMALL AUXIN UP RNA genes: Potential implications. Plant Signal. Behav. 8:15-17. doi:10.4161/psb.25283.

Rademacher, E.H., and R. Offringa. 2012. Evolutionary Adaptations of Plant AGC Kinases: From Light Signaling to Cell Polarity Regulation. Front. Plant Sci. 3:250. doi:10.3389/fpls.2012.00250.

Rayle, D.L., and R. Cleland. 1970. Enhancement of wall loosening and elongation by Acid solutions. Plant Physiol. 46:250-253. doi:10.1104/pp.46.2.250.

Rayle, D.L., and R.E. Cleland. 1992. The Acid Growth Theory of auxin-induced cell elongation is alive and well. Plant Physiol. 99:1271-1274. doi:10.1104/pp.99.4.1271. 
Ren, H., and W.M. Gray. 2015. SAUR Proteins as Effectors of Hormonal and Environmental Signals in Plant Growth. Mol. Plant. 8:1153-1164. doi:10.1016/j.molp.2015.05.003.

Roberts, D. 2013. Analysis of post-translational modifications of the blue light photoreceptor, phot1, by the CRL3 ${ }^{\mathrm{NPH} 3}$ ubiquitin ligase complex. PhD Diss. Univ. Missouri.

Roberts, D., U. V. Pedmale, J. Morrow, S. Sachdev, E. Lechner, X. Tang, N. Zheng, M. Hannink, P. Genschik, and E. Liscum. 2011. Modulation of Phototropic Responsiveness in Arabidopsis through Ubiquitination of Phototropin 1 by the CUL3-Ring E3 Ubiquitin Ligase CRL3 ${ }^{\mathrm{NPH} 3}$. Plant Cell. 23:3627-3640. doi:10.1105/tpc.111.087999.

Rockwell, N.C., Y-S. Su, J.C. Lagarias. 2006. Phytochome Structure and Signaling Mechanisms. Annu Rev Plant Biol. 57:837-858. doi:10.1146/annurev.arplant.56.032604.144208.PHYTOCHOME.

Rodriguez, L., M. Gonzalez-Guzman, M. Diaz, A. Rodrigues, A.C. IzquierdoGarcia, M. Peirats-Llobet, M.A. Fernandez, R. Antoni, D. Fernandez, J.A. Marquez, J.M. Mulet, A. Albert, and P.L. Rodriguez. 2014. C2-Domain Abscisic Acid-Related Proteins Mediate the Interaction of PYR/PYL/RCAR Abscisic Acid Receptors with the Plasma Membrane and Regulate Abscisic Acid Sensitivity in Arabidopsis. Plant Cell. 26:4802-4820.

doi:10.1105/tpc.114.129973.

Rojas-Pirela, M., D.J. Rigden, P.A. Michels, A.J. Cáceres, J.L. Concepción, and W. Quiñones. 2018. Structure and function of Per-ARNT-Sim domains and 
their possible role in the life-cycle biology of Trypanosoma cruzi. Mol. Biochem. Parasitol. 219:52-66. doi:10.1016/j.molbiopara.2017.11.002.

Rosler, J., I. Klein, and M. Zeidler. 2007. Arabidopsis fhl/fhy1 double mutant reveals a distinct cytoplasmic action of phytochrome A. Proc. Natl. Acad. Sci. 104:10737-10742. doi:10.1073/pnas.0703855104.

Sakai, T., T. Kagawa, M. Kasahara, T.E. Swartz, J.M. Christie, W.R. Briggs, M. Wada, and K. Okada. 2001. Arabidopsis nph1 and npl1: blue light receptors that mediate both phototropism and chloroplast relocation. Proc. Natl. Acad. Sci. U. S. A. 98:6969-74. doi:10.1073/pnas.101137598.

Sakai, T., T. Wada, S. Ishiguro, and K. Okada. 2000. RPT2: A Signal Transducer of the Phototropic Response in Arabidopsis. Plant Cell. 12:225-236. doi:10.1105/tpc.12.2.225.

Sakamoto, K., and W.R. Briggs. 2002. Cellular and Subcellular Localization of Phototropin 1. Plant Cell. 14:1723-1735. doi:10.1105/tpc.003293.Phot1.

Salomon, M., J.M. Christie, E. Knieb, U. Lempert, and W.R. Briggs. 2000. Photochemical and mutational analysis of the FMN-binding domains of the plant blue light receptor, phototropin. Biochemistry. 39:9401-9410. doi:10.1021/bi000585+.

Salomon, M., U. Lempert, and W. Rüdiger. 2004. Dimerization of the plant photoreceptor phototropin is probably mediated by the LOV1 domain. FEBS Lett. 572:8-10. doi:10.1016/j.febslet.2004.06.081.

Sampedro, J., and D.J. Cosgrove. 2005. The expansin superfamily. Genome Biol. 6:242. doi:10.1186/gb-2005-6-12-242. 
Sancar, A. 2004. Photolyase and cryptochrome blue-light photoreceptors. Adv. Protein Chem. 69:73-100. doi:10.1016/S0065-3233(04)69003-6.

Sandberg, W.S., P.M. Schlunk, H.B. Zabin, and T.C. Tenvilliger. 1995.

Relationship between in vivo and in vitro measures of function and stability of a protein. Biochemistry. 34:11970-11978.

Sanders, D., J. Pelloux, C. Brownlee, and J.F. Harper. 2002. Calcium at the crossroads of signaling. Plant Cell. 14 Suppl:S401-S417. doi:10.1105/tpc.002899.

Santner, A.A., and J.C. Watson. 2006. The WAG1 and WAG2 protein kinases negatively regulate root waving in Arabidopsis. Plant J. 45:752-764. doi:10.1111/j.1365-313X.2005.02641.x.

Sawa, S., M. Ohgishi, H. Goda, K. Higuchi, Y. Shimada, and S. Yoshida. 2002. The HAT2 gene, a member of the HD-Zip gene family, isolated as an auxin inducible gene by DNA microarray screening, affects auxin response in Arabidopsis. The Plant Journal. 32:1011-1022.

Schepens, I., H.E. Boccalandro, C. Kami, J.J. Casal, and C. Fankhauser. 2008. PHYTOCHROME KINASE SUBSTRATE4 Modulates PhytochromeMediated Control of Hypocotyl Growth Orientation. Plant Physiol. 147:661671. doi:10.1104/pp.108.118166.

Sedbrook, J.C., K.L. Carroll, K.F. Hung, P.H. Masson, and C.R. Somerville. 2002. The Arabidopsis SKU5 Gene Encodes an Extracellular Glycosyl Phosphatidylinositol-Anchored Glycoprotein Involved in Directional Root Growth. Plant Cell. 14:1635-1648. doi:10.1105/tpc.002360.1996. 
Shaner, N.C., R.E. Campbell, P. a Steinbach, B.N.G. Giepmans, A.E. Palmer, and R.Y. Tsien. 2004. Improved monomeric red, orange and yellow fluorescent proteins derived from Discosoma sp. red fluorescent protein. Nat. Biotechnol. 22:1567-72. doi:10.1038/nbt1037.

Short, T.W., and W.R. Briggs. 1990. Characterization of a Rapid, Blue LightMediated Change in Detectable Phosphorylation of a Plasma Membrane Protein from Etiolated Pea (Pisum sativum L.) Seedlings. Plant Physiol. 92:179-185. doi:10.1104/pp.92.1.179.

Spartz, A.K., S.H. Lee, J.P. Wenger, N. Gonzalez, D. Inzé, W.A. Peer, A.S. Murphy, P.J. Overvoorde, and M. William. 2012. The SAUR19 subfamily of SMALL AUXIN UP RNA genes promote cell expansion. The Plant Journal. 70:978-990. doi:10.1111/j.1365-313X.2012.04946.x.The.

Spartz, A.K., V.S. Lor, H. Ren, N.E. Olszewski, N.D. Miller, G. Wu, E.P. Spalding, and W.M. Gray. 2017. Constitutive Expression of Arabidopsis SMALL AUXIN UP RNA19 ( SAUR19) in Tomato Confers Auxin-Independent Hypocotyl Elongation. Plant Physiol. 173:1453-1462. doi:10.1104/pp.16.01514.

Spartz, A.K., H. Ren, M.Y. Park, K.N. Grandt, S.H. Lee, A.S. Murphy, M.R. Sussman, P.J. Overvoorde, and W.M. Gray. 2014. SAUR Inhibition of PP2CD Phosphatases Activates Plasma Membrane H+-ATPases to Promote Cell Expansion in Arabidopsis. Plant Cell. 26:2129-2142.

doi:10.1105/tpc.114.126037.

Staswick, P.E., B. Serban, M. Rowe, I. Tiryaki, M.T. Maldonado, M.C. 
Maldonado, and W. Suza. 2005. Characterization of an Arabidopsis Enzyme Family That Conjugates Amino Acids to Indole-3-Acetic Acid. Plant Cell. 17:616-627. doi:10.1105/tpc.104.026690.

Staswick, P.E., I. Tiryaki, and M.L. Rowe. 2002. Jasmonate Response Locus JAR1 and Several Related Arabidopsis Genes Encode Enzymes of the Firefly Luciferase Superfamily That Show Activity on Jasmonic, Salicylic, and Indole-3-Acetic Acids in an Assay for Adenylation. Plant Cell. 14:1405-1415. doi:10.1105/tpc.000885.defect.

Stogios, P.J., G.S. Downs, J.J.S. Jauhal, S.K. Nandra, and G.G. Privé. 2005. Sequence and structural analysis of BTB domain proteins. Genome Biol. 6:R82. doi:10.1186/gb-2005-6-10-r82.

Stone, B.B., E.L. Stowe-Evans, R.M. Harper, R.B. Celaya, K. Ljung, G. Sandberg, and E. Liscum. 2008. Disruptions in AUX1-dependent auxin influx alter hypocotyl phototropism in Arabidopsis. Mol. Plant. 1:129-44. doi:10.1093/mp/ssm013.

Stowe-Evans, E.L., R.M. Harper, A. Motchoulski, and E. Liscum. 1998. NPH4, a conditional modulator of auxin-dependent differential growth responses in Arabidopsis. Plant Physiol. 118:1265-75.

Stowe-Evans, E.L., D.R. Luesse, and E. Liscum. 2001. The enhancement of phototropin-induced phototropic curvature in Arabidopsis occurs via a photoreversible phytochrome A-dependent modulation of auxin responsiveness. Plant Physiol. 126:826-834. doi:10.1104/pp.126.2.826. Strader, L.C., and Y. Zhao. 2016. Auxin perception and downstream events. 
Curr. Opin. Plant Biol. 33:8-14. doi:10.1016/j.pbi.2016.04.004.

Stringer, D.K., and R.C. Piper. 2011. Terminating protein ubiquitination: Hasta la vista, ubiquitin. Cell Cycle. 10:3067-3071. doi:10.4161/cc.10.18.17191.

Suetsugu, N., F. Mittmann, G. Wagner, J. Hughes, and M. Wada. 2005. A chimeric photoreceptor gene, NEOCHROME, has arisen twice during plant evolution. Proc. Natl. Acad. Sci. U. S. A. 102:13705-13709. doi:10.1073/pnas.0504734102.

Suetsugu, N., A. Takemiya, S.-G. Kong, T. Higa, A. Komatsu, K. Shimazaki, T. Kohchi, and M. Wada. 2016. RPT2/NCH1 subfamily of NPH3-like proteins is essential for the chloroplast accumulation response in land plants. Proc. Natl. Acad. Sci. 113:10424-10429. doi:10.1073/pnas.1602151113.

Sullivan, J.A., and X.W. Deng. 2003. From seed to seed: The role of photoreceptors in Arabidopsis development. Dev. Biol. 260:289-297. doi:10.1016/S0012-1606(03)00212-4.

Sullivan, S., E. Kaiserli, T. Tseng, and J.M. Christie. 2010. Subcellular localization and turnover of Arabidopsis phototropin 1. Plant Signal. Behav. 5:184-186. doi:10.1105/tpc.109.067876.e.

Sullivan, S., C.E. Thomson, D.J. Lamont, M. a Jones, and J.M. Christie. 2008. In vivo phosphorylation site mapping and functional characterization of Arabidopsis phototropin 1. Mol. Plant. 1:178-94. doi:10.1093/mp/ssm017. Takahashi, K., K. -I. Hayashi, and T. Kinoshita. 2012. Auxin Activates the Plasma Membrane H+-ATPase by Phosphorylation during Hypocotyl Elongation in Arabidopsis. Plant Physiol. 159:632-641. doi:10.1104/pp.112.196428. 
Takemiya, A., S. Inoue, and M. Doi. 2005. Phototropins promote plant growth in response to blue light in low light environments. Plant Cell. 17:1120-1127. doi:10.1105/tpc.104.030049.2.

Tatematsu, K., S. Kumagai, H. Muto, A. Sato, M.K. Watahiki, R.M. Harper, E. Liscum, and K.T. Yamamoto. 2004. MASSUGU2 Encodes Aux/IAA19, an Auxin-Regulated Protein That Functions Together with the Transcriptional Activator NPH4/ARF7 to Regulate Differential Growth Responses of Hypocotyl and Formation of Lateral Roots in Arabidopsis thaliana. Plant Cell. 16:379-393. doi:10.1105/tpc.018630.

Thomann, A., E. Lechner, M. Hansen, E. Dumbliauskas, Y. Parmentier, J. Kieber, B. Scheres, and P. Genschik. 2009. Arabidopsis CULLIN3 genes regulate primary root growth and patterning by ethylene-dependent and independent mechanisms. PLoS Genet. 5:e1000328.

doi:10.1371/journal.pgen.1000328.

Till, B.J., S.H. Reynolds, E.A. Greene, C.A. Codomo, L.C. Enns, J.E. Johnson, C. Burtner, A.R. Odden, K. Young, N.E. Taylor, J.G. Henikoff, L. Comai, and S. Henikoff. 2003. Large-Scale Discovery of Induced Point Mutations With High-Throughput TILLING. Genome Res. 524-530. doi:10.1101/gr.977903.1.

Titapiwatanakun, B., J.J. Blakeslee, A. Bandyopadhyay, H. Yang, J. Mravec, M. Sauer, Y. Cheng, J. Adamec, A. Nagashima, M. Geisler, T. Sakai, J. Friml, W.A. Peer, and A.S. Murphy. 2009. ABCB19/PGP19 stabilises PIN1 in membrane microdomains in Arabidopsis. Plant J. 57:27-44. 
doi:10.1111/j.1365-313X.2008.03668.x.

Tokutomi, S., D. Matsuoka, and K. Zikihara. 2008. Molecular structure and regulation of phototropin kinase by blue light. Biochim. Biophys. Acta Proteins Proteomics. 1784:133-142. doi:10.1016/j.bbapap.2007.09.010.

Treml, B.S., S. Winderl, R. Radykewicz, M. Herz, G. Schweizer, P. Hutzler, E. Glawischnig, R.A. Torres Ruiz. 2005. The gene ENHANCER OF PINOID controls cotyledon development in the Arabidopsis embryo. Development. 132:4063-4074. doi:10.1242/dev.01969.

Tseng, T.-S., and W.R. Briggs. 2010. The Arabidopsis ren1-1 Mutation Impairs Dephosphorylation of Phot2, Resulting in Enhanced Blue Light Responses. Plant Cell. 22:392-402. doi:10.1105/tpc.109.066423.

Tsuchida-Mayama, T., M. Nakano, Y. Uehara, M. Sano, N. Fujisawa, K. Okada, and T. Sakai. 2008. Mapping of the phosphorylation sites on the phototropic signal transducer, NPH3. Plant Sci. 174:626-633.

doi:10.1016/j.plantsci.2008.03.018.

Tsuchida-Mayama, T., T. Sakai, A. Hanada, Y. Uehara, T. Asami, and S. Yamaguchi. 2010. Role of the phytochrome and cryptochrome signaling pathways in hypocotyl phototropism. Plant J. 62:653-662. doi:10.1111/j.1365-313X.2010.04180.x.

Ulmasov, T., G. Hagen, and T.J. Guilfoyle. 1997. ARF1, a Transcription Factor That Binds to Auxin Response Elements. Science. 276:1865-1868.

Urano, D., J.-G. Chen, J.R. Botella, and A.M. Jones. 2013. Heterotrimeric G protein signalling in the plant kingdom. Open Biol. 3:120186-120186. 
doi:10.1098/rsob.120186.

Wan, Y.-L., W. Eisinger, D. Ehrhardt, U. Kubitscheck, F. Baluska, and W. Briggs. 2008. The subcellular localization and blue-light-induced movement of phototropin 1-GFP in etiolated seedlings of Arabidopsis thaliana. Mol. Plant. 1:103-17. doi:10.1093/mp/ssm011.

Wan, Y., J. Jasik, L. Wang, H. Hao, D. Volkmann, D. Menzel, S. Mancuso, F. Baluska, and J. Lin. 2012. The Signal Transducer NPH3 Integrates the Phototropin1 Photosensor with PIN2-Based Polar Auxin Transport in Arabidopsis Root Phototropism. Plant Cell. 24:551-565. doi:10.1105/tpc.111.094284.

Went, F.W., and K. V. Thimann. 1937. Phytohormones.

Westfall, C.S., J. Herrmann, Q. Chen, S. Wang, and J.M. Jez. 2010. Modulating plant hormones by enzyme action. Plant Signal. Behav. 5:1607-1612. doi:10.4161/psb.5.12.13941.

Whippo, C.W., and R.P. Hangarter. 2003. Second Positive Phototropism Results from Coordinated Co-Action of the Phototropins and Cryptochromes1. Plant Physiol. 132:1499-1507. doi:10.1104/pp.102.018481.).

Whippo, C.W., and R.P. Hangarter. 2004. Phytochrome modulation of blue-lightinduced phototropism. Plant, Cell Environ. 27:1223-1228.

doi:10.1111/j.1365-3040.2004.01227.x.

Willige, B.C., S. Ahlers, M. Zourelidou, I.C.R. Barbosa, E. Demarsy, M. Trevisan, P. a Davis, M.R.G. Roelfsema, R. Hangarter, C. Fankhauser, and C. Schwechheimer. 2013. D6PK AGCVIII Kinases Are Required for Auxin 
Transport and Phototropic Hypocotyl Bending in Arabidopsis. Plant Cell. 1:1-16. doi:10.1105/tpc.113.111484.

Woo, O.-G., S.-H. Kim, S.K. Cho, S.-H. Kim, H.N. Lee, T. Chung, S.W. Yang, and J.-H. Lee. 2018. BPH1, a novel substrate receptor of CRL3, plays a repressive role in ABA signal transduction. Plant Mol. Biol. 96:593-606. doi:10.1007/s11103-018-0717-x.

Wu, J., S. Liu, Y. He, X. Guan, X. Zhu, L. Cheng, J. Wang, and G. Lu. 2012. Genome-wide analysis of SAUR gene family in Solanaceae species. Gene. 509:38-50. doi:10.1016/j.gene.2012.08.002.

Yennawar, N.H., L.-C. Li, D.M. Dudzinski, A. Tabuchi, and D.J. Cosgrove. 2006. Crystal structure and activities of EXPB1 (Zea m 1), a beta-expansin and group-1 pollen allergen from maize. Proc. Natl. Acad. Sci. U. S. A. 103:14664-14671. doi:10.1073/pnas.0605979103.

Yuan, S., Y. Wu, and D.J. Cosgrove. 2001. A Fungal Endoglucanase with Plant Cell Wall Extension Activity. Plant Physiol. 127:324-333. doi:10.1104/pp.127.1.324.

Zandomeni, K., and P. Schopfer. 1993. Reorientation of microtubules at the outer epidermal wall of maize coleoptiles by phytochrome, blue-light photoreceptor, and auxin. Protoplasma. 173:103-112. doi:10.1007/BF01378999.

Zazímalová, E., A.S. Murphy, H. Yang, K. Hoyerová, and P. Hosek. 2010. Auxin transporters--why so many? Cold Spring Harb. Perspect. Biol. 2:a001552. doi:10.1101/cshperspect.a001552. 
Zhang, L., L. Du, C. Shen, Y. Yang, and B.W. Poovaiah. 2014. Regulation of plant immunity through ubiquitin-mediated modulation of $\mathrm{Ca} 2+$-calmodulinAtSR1/CAMTA3 signaling. Plant J. 78:269-281. doi:10.1111/tpj.12473.

Zhang, X.S., and S.D. ONeill. 1993. Ovary and Gametophyte Development Are Coordinately Regulated by Auxin and Ethylene following Pollination. Plant Cell. 5:403-418. doi:10.1105/tpc.5.4.403.

Zhao, X., Y.-L. Wang, X.-R. Qiao, J. Wang, L.-D. Wang, C.-S. Xu, and X. Zhang. 2013. Phototropins Function in High-Intensity Blue Light-Induced Hypocotyl Phototropism in Arabidopsis by Altering Cytosolic Calcium. Plant Physiol. 162:1539-1551. doi:10.1104/pp.113.216556.

Zhao, Y. 2010. Auxin biosynthesis and its role in plant development. Annu Rev Plant Biol. 61:49-64. doi:10.1146/annurev-arplant-042809-112308.Auxin.

Zourelidou, M., I. Muller, B.C. Willige, C. Nill, Y. Jikumaru, H. Li, and C. Schwechheimer. 2009. The polarly localized D6 PROTEIN KINASE is required for efficient auxin transport in Arabidopsis thaliana. Development. 136:627-636. doi:10.1242/dev.028365. 


\section{Vita}

Johanna Morrow was born on the $20^{\text {th }}$ day of December in the year 1975 in St. Louis, Missouri. She grew up in St. Louis county attending Holy Infant Elementary School and Ursuline Academy for High School. Upon completion of high school Johanna attended the University of Missouri and received a Bachelor of Arts in Biological Sciences in 1999. She then worked in industry for a few years prior to beginning a job as research specialist at MU for Dr. Mannie Liscum. While working as a research specialist she completed her Masters Degree in Heath Care Management (MHA) in 2006 at the University of Missouri. In 2012, Johanna worked as a research specialist for 6 months for Dr. Paula McSteen before entering the Ph.D. program at MU in the Biological Sciences department. While at MU she was active in the Interdisciplinary Plant Group Student Group as (IPG-SPO) well as the Bond Life Science Post-Doc and Graduate Student Association (PSC-PGSA). Additionally, Johanna was able to mentor many undergraduate students through her role as Graduate Student Coordinator for FRIPS (Freshman Research in Plant Sciences), as well as teach students through her role as Teaching Assistant for a variety of courses. In June 2018 Johanna obtained her Ph.D. mentored by Dr. Mannie Liscum. Johanna has accepted a position as Visiting Assistant Professor of Biology at Westminster College in Fulton, MO starting in August 2018. 